\title{
$E N-77-C-02-4299$
}

\section{MASTER}

KILOWATT ISOTOPE

POWER SYSTEM

COMPONENT TEST REPORT FOR THE

ELECTRIC HEAT SOURCE ASSEMBLY

PREPARED BY

TELEDYNE ENERGY SYSTEMS

UNDER

SUBCONTRACT 282566
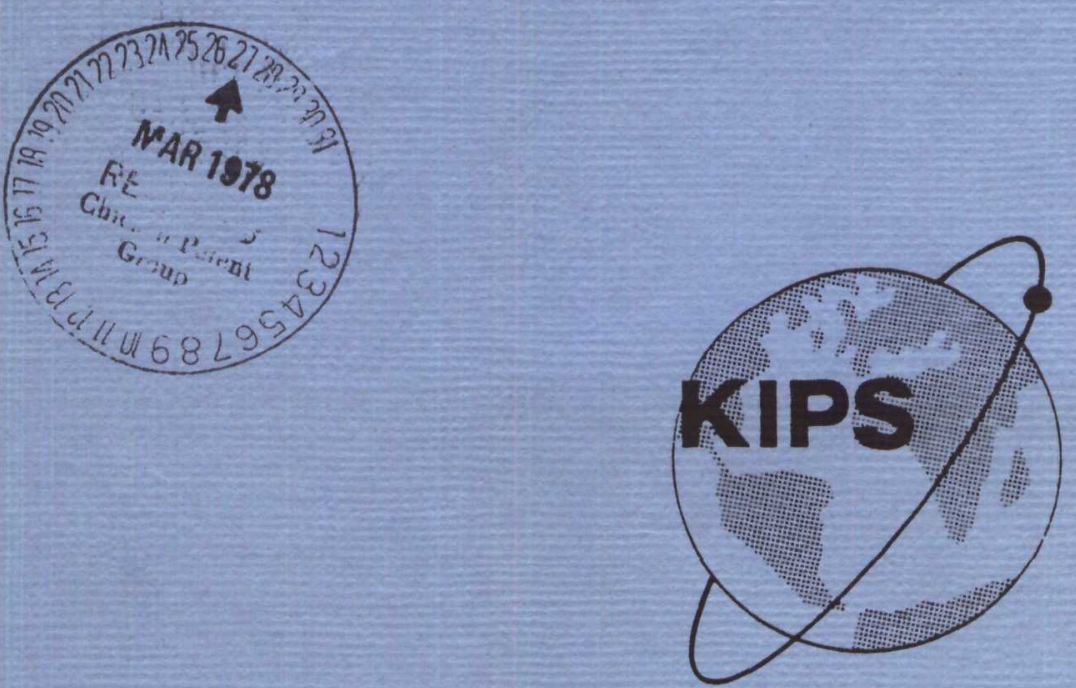

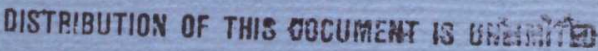

\section{Sundstrand Energy Systems}

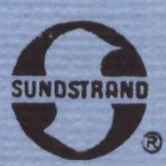




\section{DISCLAIMER}

This report was prepared as an account of work sponsored by an agency of the United States Government. Neither the United States Government nor any agency Thereof, nor any of their employees, makes any warranty, express or implied, or assumes any legal liability or responsibility for the accuracy, completeness, or usefulness of any information, apparatus, product, or process disclosed, or represents that its use would not infringe privately owned rights. Reference herein to any specific commercial product, process, or service by trade name, trademark, manufacturer, or otherwise does not necessarily constitute or imply its endorsement, recommendation, or favoring by the United States Government or any agency thereof. The views and opinions of authors expressed herein do not necessarily state or reflect those of the United States Government or any agency thereof. 


\section{DISCLAIMER}

Portions of this document may be illegible in electronic image products. Images are produced from the best available original document. 


\section{NOTICE}

"This document was prepared as an account of work sponsored by the United States Government. Neither the United States nor the United States Energy Research \& Development Administration, nor any of their employees, nor any of their contractors, subcontractors, or their employees, make any warranty, express or Implied, or assumes any legal liablifty or responsibility for the accuracy, completeness or usefulness of any information, apparatus, product or process disclosed, or represents that its use would not infringe privately-owned rights". 
C. Alexander

A. Bartholomew

R. Beard

W. Boyes

R. Brouns (3)

J. Daniel

F. Dieringer

Director, Reactor Programs

Division, Chicago Operations Office

T. Dobry (G. Ogburn)

M. Eck

E. Gallagher

J. Griffo

S. Gorland

R. Harner

E. Johnson

C. Jund (R. Widby)

D. Kenney

H. Kling

M. Langsam

R. Lawson

J. Lombardo

E. Mahefkey (B. McFadden)

R. Migra

R. Morrow

J. Mullin

B. Raab

H. Sampson

A. Schaffhauser

A. Schock

J. Sholtis

C. Tarr

N. Thielke 
nepont No. ASR 1504-10

oAte February 2, 1978.

AEVISION

OATE

FILE REF.

SUNDSTRAND AVIATION

DIVISION OF SUNDSTRANO CORPORATION

ROCKFORD, ILLINOIS

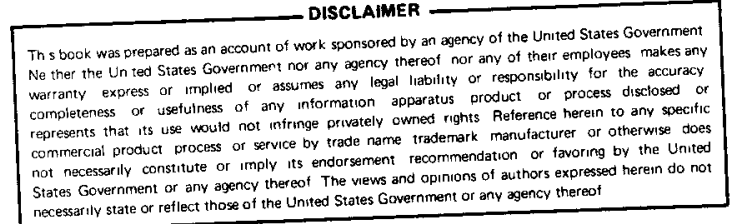

ENGINEERING REPORT

TELEDYNE ENERGY SYSTEMS

PERFORMANCE TEST ANALYSIS FOR

THE KILOWATT ISOTOPE POWER SUPPLY (KIPS)

ELECTRIC HEAT SOURCE ASSEMBLY (EHSA)

DISTRIBUTION OF THIS DOCUMCH is URth

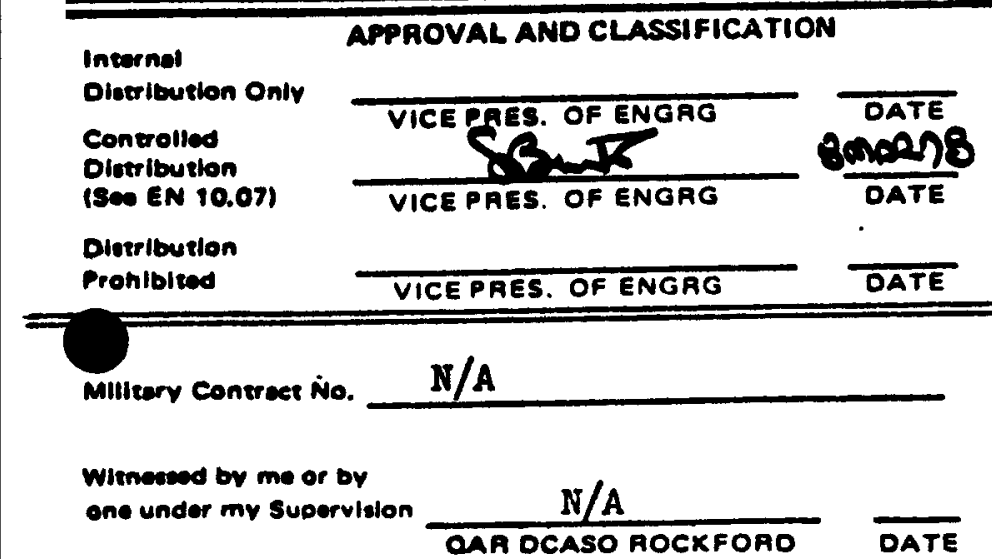

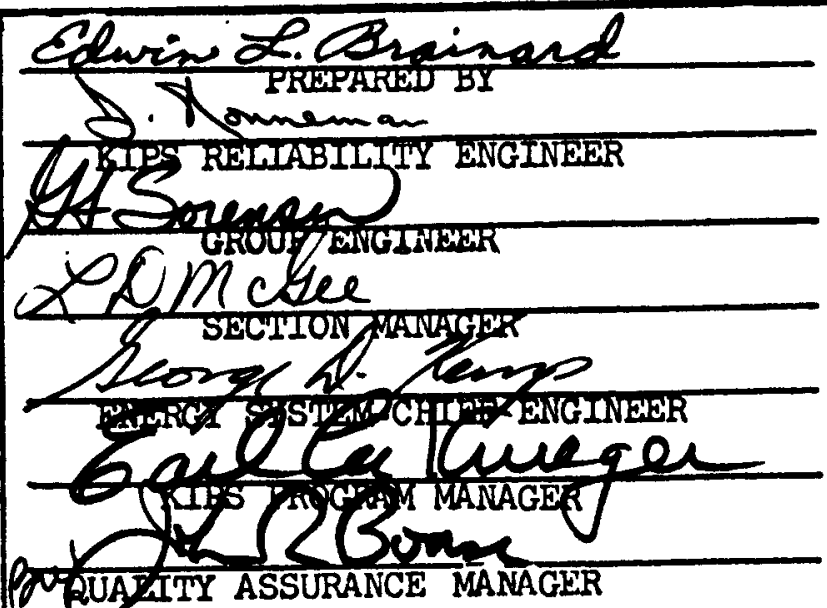




\section{TABLE OF CONTENTS}

Paragraph

1.0

2.0

3.0

4.0

APPENDIX I
PURPOSE

CONCLUSIONS

TEST PROCEDURE

TEST RESULTS
Page

1

2

3

3

TELEDYNE ENERGY SYSTEMS, EHSA ACCEPTANCE TEST PROCEDURE

NUMBER KIPS 1020304

4

APPENDIX II TELEDYNE ENERGY SYSTEMS, EHSA PERFORMANCE TEST ANALYSIS

NUMBER KIPS-DKD-086 
1.0

\section{PURPOSE}

The purpose of the acceptance testing was to demonstrate that the Electrical Heat Source Assembly (EHSA) has completed sufficient testing to satisfy the requirements set forth within the Kilowatt Isotope Power System (KIPS) Component Test Procedure (\#KIPS1020304) for the Electrical Heat Source Assembly.

This document, one of a series, presents the results of the Acceptance Testing/Analys is conducted by Teledyne Energy Systems on the EHSA. 
SUNDSTRAND

ADVANCE TECHNOLOGY OPERATIONS
TEST TITLE

TELEDYNE PERFORMANCE

TEST ANALYSIS FOR EHSA
AFR $\quad 1504-10$

PAGE 2

2.0

CONCLUSIONS

The Model Number ORCIA3AOI Electrical Heat Source

Assembly (EHSA) for the Kilowatt Isotope Power

System (KIPS) has completed testing as set forth

within Teledyne Energy System's Test Procedure

KIPS 1020304 and is qualified to be

used for further testing with the complete system.

100369 
SUNDSTRAND ADVANCE TECHNOLOGY OPERATIONS
TEST TITLE

TELEDYNE PERFORMANCE

TEST ANALYSIS FOR EHSA
AER 1504-11

PAGE

3.0 TEST PROCEDURE

As shown in Appendix I.

4.0 RESULTS

As shown in Appendix II. 
APPENDIX I

TELEDYNE ENERGY SYSTEMS

EHSA ACCEPTANCE TEST PROCEDURE

NUMBER KIPS1020304

(SUNDSTRAND TEST PROCEDURE NUMBER 409) 
AER 1504-10

Page 1

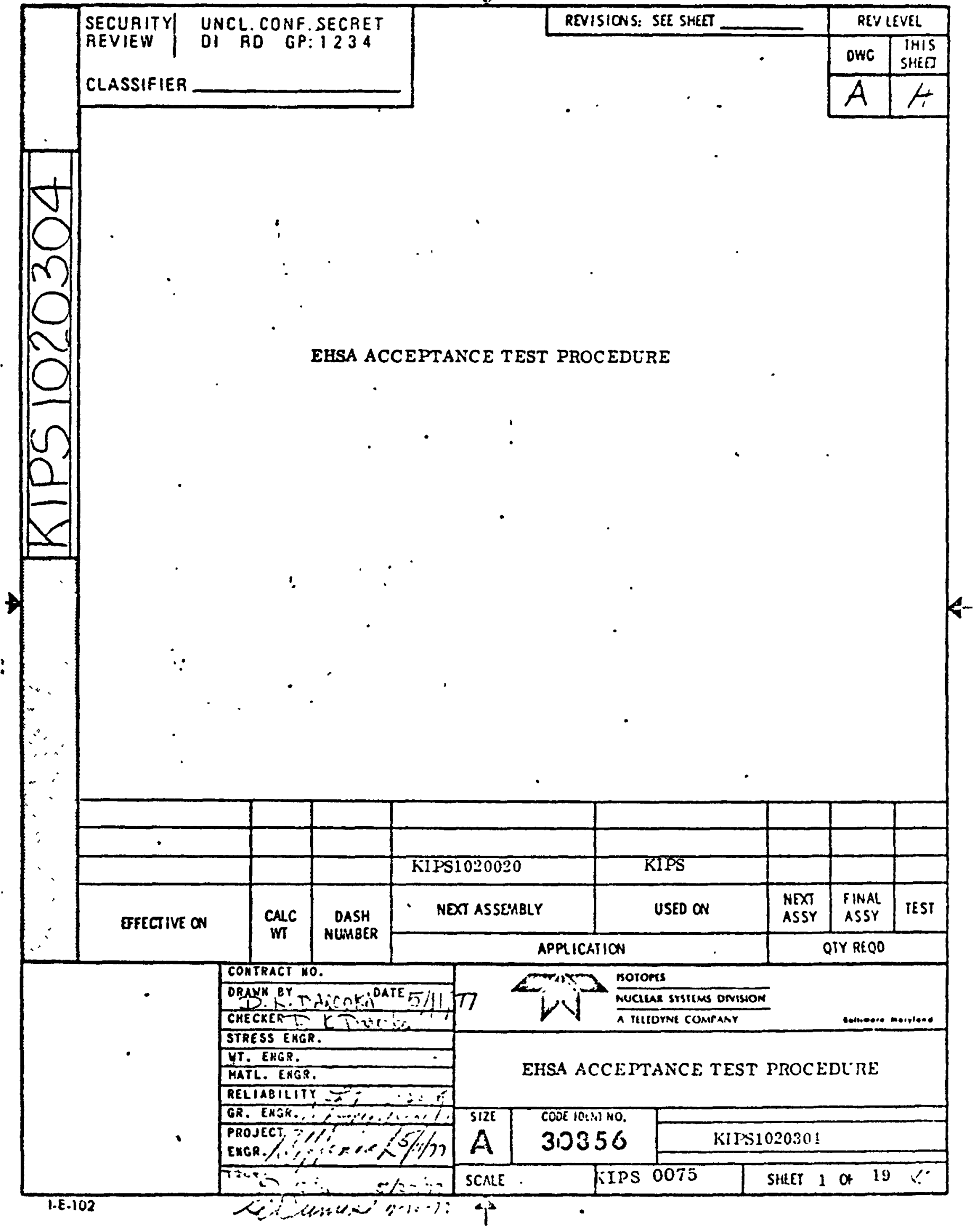


AER 1504-10

APPENDIX I

Page 2

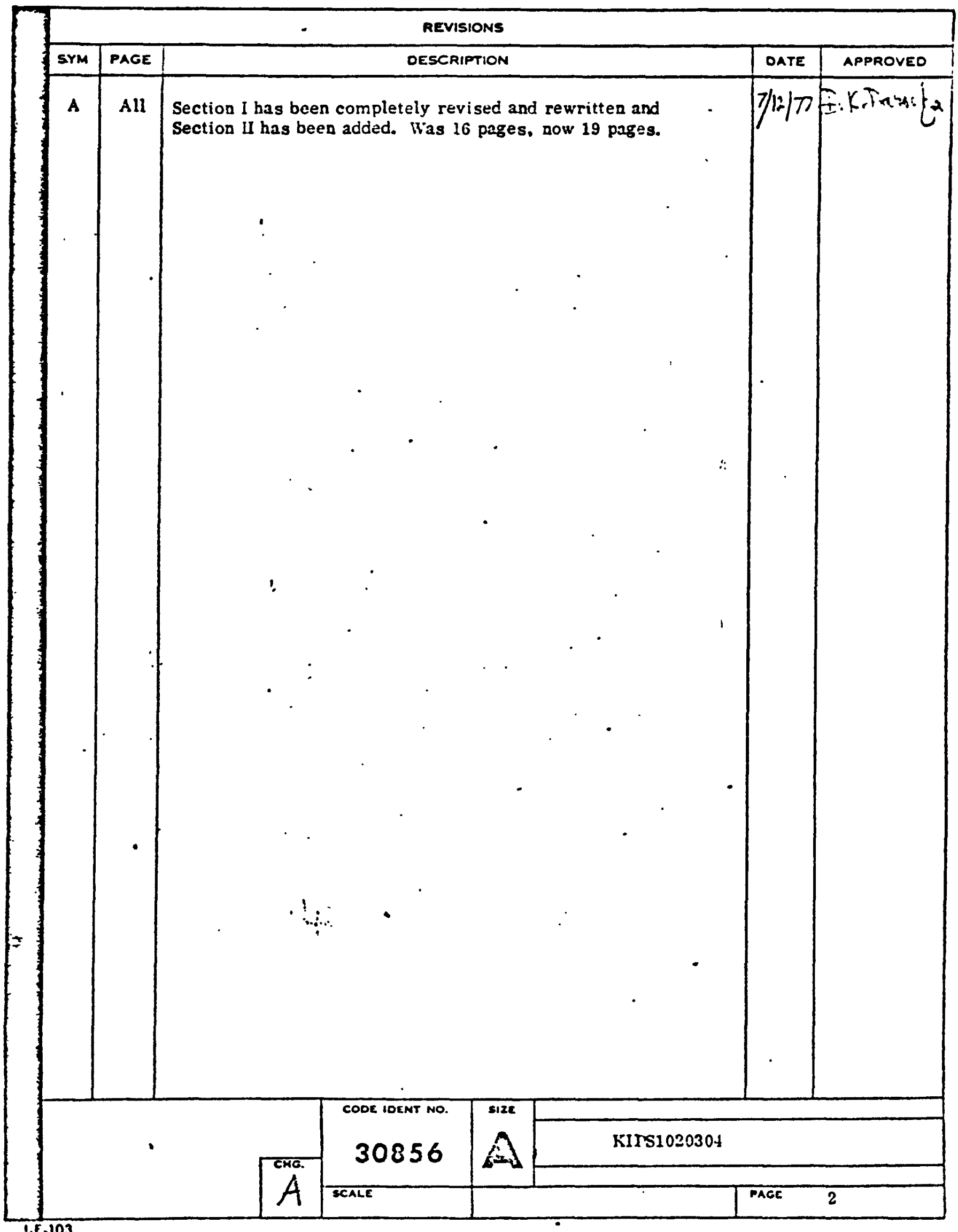




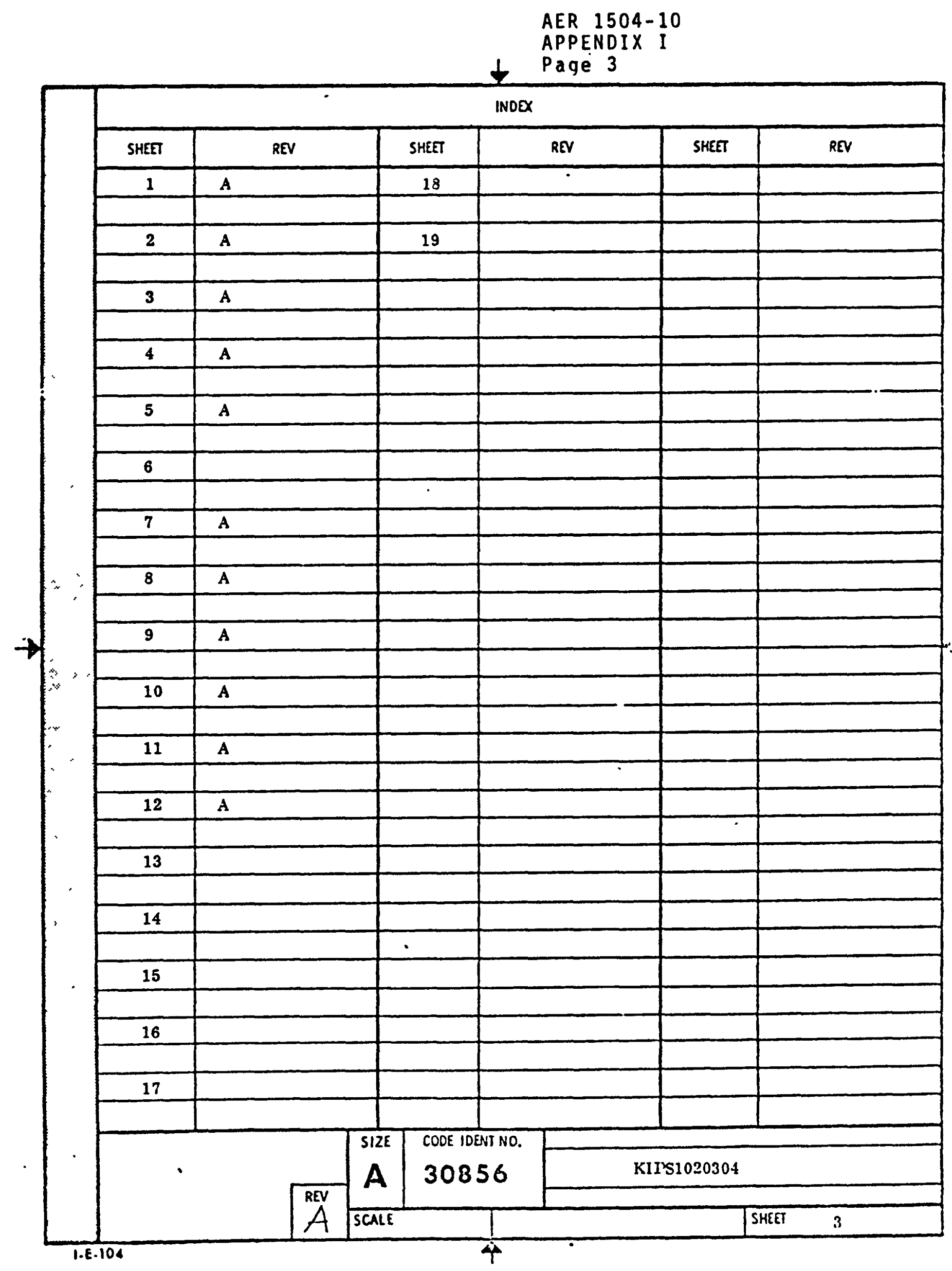




\section{TABLE OF CONTENTS}

$\begin{array}{llr}1.0 & \text { Objective and Scope } & 5 \\ 2.0 & \text { Applicable Documents } & 5 \\ 3.0 & \text { Facilities, Equipments and Niaterials } & 5 \\ 4.0 & \text { Warnings and Cautions } & 7 \\ & \text { SECTION I } & 7 \\ 5.0 & \text { Test Procedure for KIPS1020020-039 } & 7 \\ 6.0 & \text { Shutdown Procedure } & 10 \\ 7.0 & \text { Storage } & 10 \\ 3.0 & \text { SECTION II } & 11 \\ & \text { Acceptance Tests for EHSA } & 11\end{array}$


1.0 OBJECTIVE A VD SCOPE

1.1 Obiective

The objective of this document is to describe the procedure for conducting the performance and acceptance tests on the electrical heat source assemblies (EHSA's). The results of the tests on the first EHSA (KIPS1020020-039) "1ill be used in evaluating its design, whereas those of the tests on the subsequent EHSA's will be used for functional checkout.

\subsection{Scope}

Section I of this procedure applies to the tests to be performed on electrical heat source assembly (KIPS1020020-039). These tests will be conducted inside the thermal vacuum chamber and will be performed with only liquid phase of the circulating fluid in the EHSA. The measurements will include temperature distribution and electrical circuit resistance. The tests on the subsequent EHSA (KIPS1020020-009, 019, 029) will be performed as per the procedure of Section II, and at the outgassing station.

2.0 APPLICABLE DOCUNIENTS

2.1 KIPS1020020 - Electrical Heat Source Assembly

2.2 PS0600003 - Instrumentation Thermcouple Installation

2.3 KIPS1030000 - Power Supply Console

2.4500 A1401108 - Condenser Assembly

2.5 KIPS1020305 - Cold Leak and Outgassing Procedure EHSA

\section{0 FACILITIES, EQUIPNIENTS AND MATERLA LS}

\section{$3.1 \quad$ Facilities}

3.1.1 Thermal Vacuum Chamber

3.1.2 Cooling water supply

\subsection{Equipments}

Equivalent or better equipment may be substituted.

3.2.1 Circulaling Bath - Haake HT22 and accessories.

\begin{tabular}{|c|c|c|c|}
\hline & \multirow{2}{*}{$\begin{array}{c}\text { SIZE } \\
A\end{array}$} & \multirow{2}{*}{$\begin{array}{l}\text { CODE IDENT NO. } \\
30356\end{array}$} & \multirow[b]{2}{*}{ KIPS102030t } \\
\hline & & & \\
\hline$A$ & $\overline{S C A L E}$ & & SHEET \\
\hline
\end{tabular}


AER 1504-10

APPENDIX I

Page 6

3.2.2 Flow Meters - Brooks Sho-Rate Model \#1355, tube \# R-6-15-B with tantalum loat.

3.2.3 Differential Pressure Gauge - Ashcroft Gauge Model 1125 0-30 psi and 0-60 psi.

3.2.4 Pump - Procon Rotary Vane Type Model 2504.

3.2.5 Digital Thermometer - Type K United System $590 \mathrm{KF}$

3.2.6 Thermocouple Switch - Thermcelectric Type K, 32 positions.

3. 2. 7 Pressure Gauge - U.S. Gauge Model No. 19003 (100 psig)

3.2.8 Potentiometer with compensation capabillty, Model \#2745, Honeywell

3.2.9 Wattmeter - 0-3000 watt ranze, Weston

3.2.10 Variac - 0-120 V AC, 30 amps

3.2.11 Digital Multimeter - range 0-120 VAC, Keithley 190

3.2.12 Multimeter - Simpson 260

3.2.13 Wheatstone Bridge - Capable of measuring heater element resistance to four significant figures.

3.2.14 Megohm Briage - General Radio Co., Model \#1644-A

3.2.15 Condenser Assembly - 500A1401108 (two)

3.2. 16 Power Supply 0 - 120V, 50 amps AC

3.3 Materials

3.3.1 Dowtherm A or equivalent llquid - Eastman Phenyl-ether-Bl phenyl eutectlc $\therefore$ \& $\therefore$. . . .

3.3.2 Insulation - Fiberglas

3.3.3 Distilled Water - Approximately $100000 \Omega \mathrm{cm}$ resistance.

3.3.4 Alcohol Ethyl Denatured

3.3.5 Argon - Compressed gas MIIL-A-18455 or evaporated liquid

3. 3. 6 Thermocouple Wires - 24 AWG Type $\mathrm{K} \mathrm{Cb} / \mathrm{Al}$ Teflon insulated.

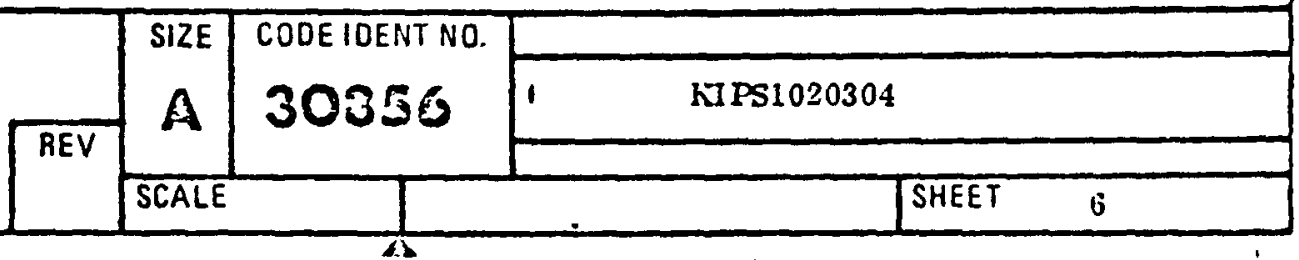




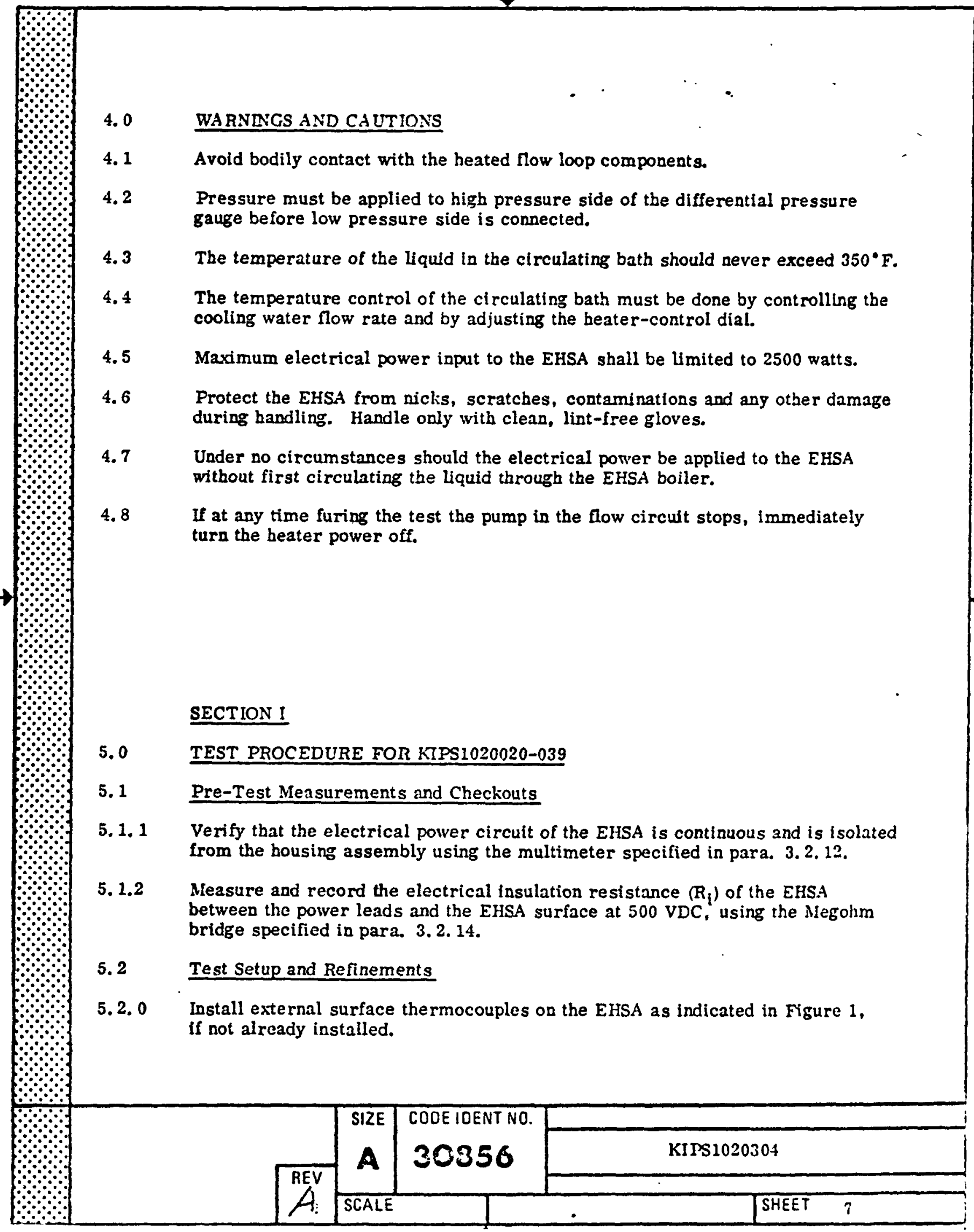


5.2.1 Place the EHSA as assembled in KIPSi020020-039 inside the the rmal vacuum chamber. Connect the electrical power supply as indicated in Figure 2. (If available, use EHSA Fower Supply Console hIPS1030000.) Avoid any direct contact between the wall of the thermal vacuum chamber and the EHSA.

5.2. 2 Connect the flow circuit as shown in Figure 3. Connect 0.5" EHSA boiler tubes to the now circuit.

5.2.3 Leak-check circuit by pressurizing the loop with dry argon specified in para. 3.3.5 to 40 psig and using snoop or equivalent leak detection nuid. Circulate "Dowtherm A" througi the system by pouring it into the circulating bath. Adjust the valve $=1$ and $=2$ (Figure 3 ) such that the flow rate through EHSA is in the middle of the rotameter range.

5.2.4 Calculate and record the electrical circuit resistance $\left(R_{c}\right)$ of the EHSA by the
following method:

Measure the voltage drop and the total current input while applying 80 watts power to the EHSA and calculate the circuit resistance from the following formula

$$
R_{c}=\text { (voltage drop)/total current input }
$$

5.2.5 Verify that all the visible thermocouples are intast and that all are reading appropriate temperatures.

5.2.6 Just before closing the door of the thermal vacuum chamber, open the outgassing valve on the EISA and evacuate the the rmal vacuum chamber to $1 H$ ( $2 \times 10^{-4}$ torr) or less and maintain it the re.

5.2.7 Circulate cooling water through the heat exchanger IL

\subsection{EHSA Performance Test}

5.3. 1 By controlling the valves $\$ 1$ and $\$ 2$ establish the rotameter reading at 6 (0. $0285 \mathrm{lbs} / \mathrm{sec}$ from the calibration curve Firure 4 ). Allow a faw minutes for flow to get stabilized. Verify stabilization by noting that the rotameter reading does not change over a period of 10 minutes. If necessary readjust valve $\$ 1$. System pressure should be maintained at $40 \pm \overline{5} \mathrm{psig}$.

5.3.2 Raise the temperature of the liquid to $250^{\circ} \mathrm{F}$ by adjusting the heater control of the circulating bath.

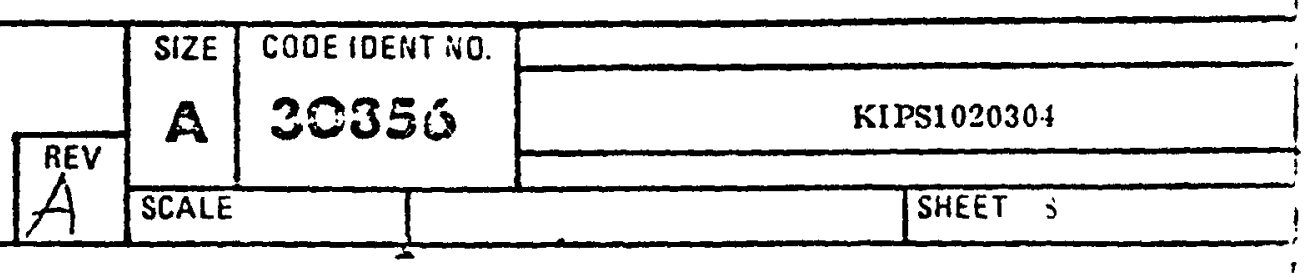




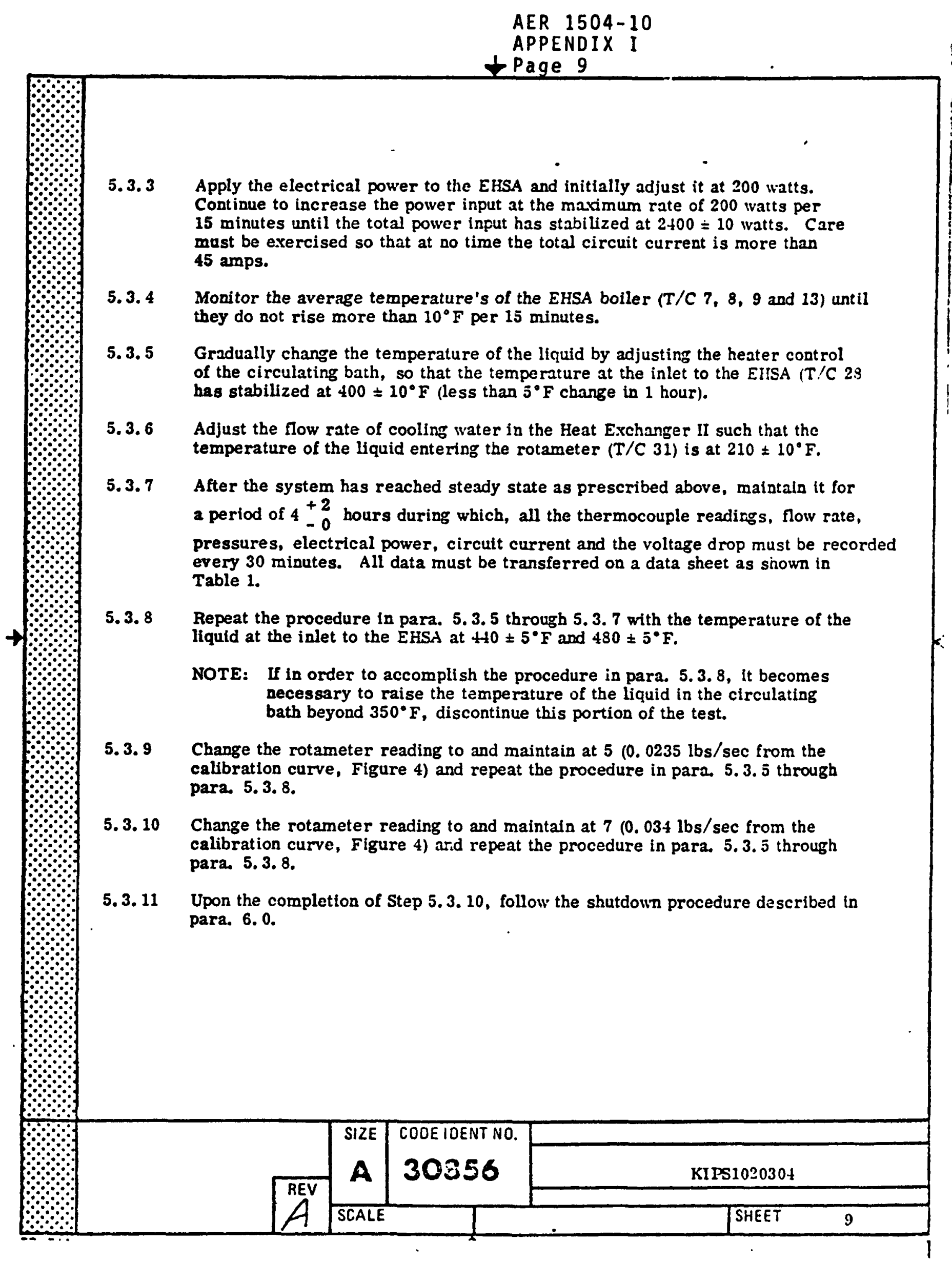




\subsection{SHUTDOWN PROCEDCRE}

6. 1 Reduce the electrical power to the EHSA by 300 watts per 15 minutes. After one bour turn off the big vacuum pump (20') on TV chamber.

6.2 After heater power is reduced to zero; increase the Dowtherm flow rate so that rotameter reads two numbers higher than the last reading.

6.3 After 45 minutes switch off all electrical heaters in the flow circuit.

6.4 Gradually increase the cooling water through Heat Exchanger II unitl T/C 31 reads less than $100^{\circ} \mathrm{F}$.

0.5 After heater block temperature has dropped to less than $600^{\circ} \mathrm{F}$, turn off the pump and circulating bath.

6. 6 Turn off the small (6') diffusion and mechanical pump on TV chamber.

6. 7 Lock the door of the TV chamber.

6.8 Backfill the TV chamber with argon specified in para. 3.3.5 to -2 in $\mathrm{Hg}$ psig.

6.9 Measure and record all the thermocouple readings, flow rate, pressure etc. , listed in Table 1.

6. 10 Measure and record the circuit resistance $\left(R_{c}\right)$ by means of voltage drop method as in para 5.2.4. Calculate and record the percentage difference (Pd) as follows:

$$
P d=\frac{R_{c}-R_{c_{t}}}{R_{c_{t}}} \times 100
$$

where,

$$
R_{c_{t}}=\text { circuit resistanie measured in para. } 5.2 .4
$$

6. 11 Measure and record the EHSA insulation resistance $\left(R_{i}\right)$ as in para. 5. 1. 2.

\subsection{STORAGE}

7.1 Upon completion of this test the EHSA unit must be stored in an inert atmosphere.

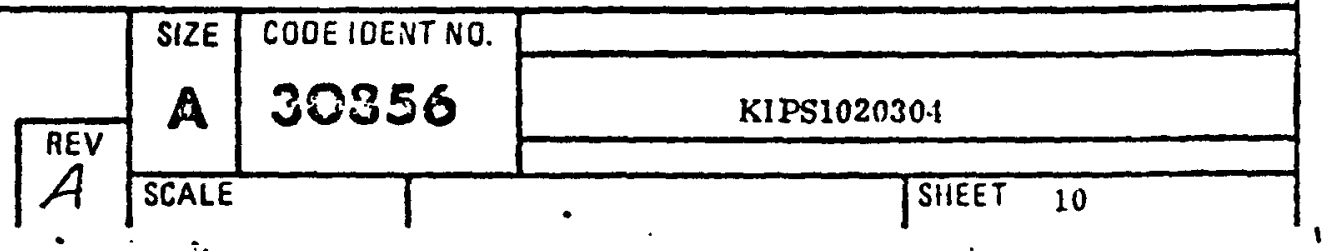




\section{SECTION II}

\subsection{ACCEPTANCE TESTS FOR EHSA}

NOTE: These tests will be performed at the outgassing station Refer to Cold Leak and Outgassing Procedure (KIPS1020305).

\section{1 Performance Checkout}

8.1.1 Upon completion of para. 6.2.17 of Cold Leak and Outgassing Procedure (!IIPS102030.5) readjust (if not already at) the Dowtherm flow rate, such that the rotameter reads 5 (0. $0234 \mathrm{lbs} / \mathrm{sec})$.

8. 1.2 Maintain the total electrical power input to the EHSA at $2400 \doteq 5$ watts,

8. 1. 3 Adjust and maintain the boiler inlet temperature at $400 \pm 5^{\circ} \mathrm{F} \mathrm{T} / \mathrm{C} 28$ by controlling the temperature of the circulating bath. If necessary, raise the temperature of the liquid entering the rotameter $(T / C 31)$ such that it remains at $210 \pm 10^{\circ} \mathrm{F}$.

8.1.4 After the beater block and boiler temperatures have stabilized such that none of the temperatures change more than $10^{\circ} \mathrm{F}$ per 30 minutes, maintain it for a period of $1 \pm 1 / 4$ hour during which, all the thermocouple readings, flow raie. pressures, electrical power, circuit current, and voltagedrop must be recorded every 30 minutes.

8.1.5 Calculate the amount of heat carried away by the boller fluid from the EHSA as follows:

$$
H=\dot{m} c_{p}\left(T_{2}-T_{1}\right) \quad 3600 / 3.412 \text { watts }
$$

where,

$$
\begin{aligned}
& \dot{m}=\text { mass flow rate of Dowtherm Ibs/sec } \\
& \mathbf{T}_{\mathbf{2}}=\text { average boiler outlet temperature }{ }^{\circ} \mathrm{F}(\mathrm{T} / \mathrm{C} 29) \\
& \mathbf{T}_{\mathbf{1}}=\text { average boiler inlet temperature " } \mathrm{F}(\mathrm{T} / \mathrm{C} 28) \\
& c_{p}=\text { average of the specific heat of Dowtherm at boiler inlet and outlet }
\end{aligned}
$$

$H$ may not be less than 2100 watts.

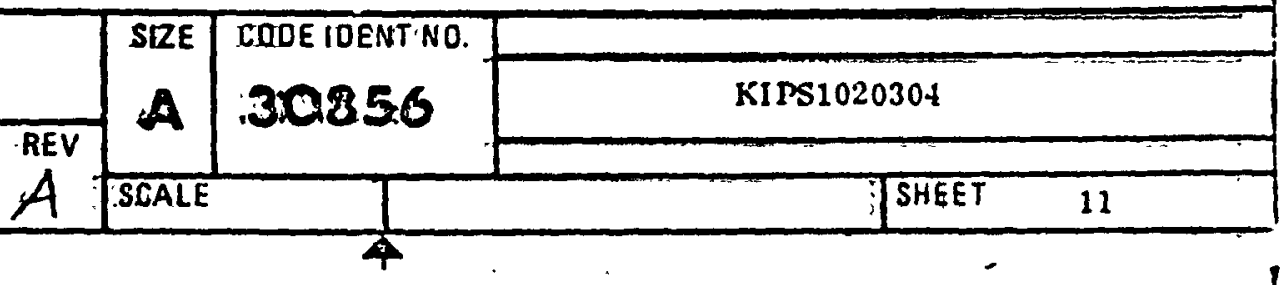


8. 1.6 Repeat the procedure in para. 8.1.1 through 8.1 .5 for the rotameter reading of $6(0.0285 \mathrm{lbs} / \mathrm{sec})$ and $7(0.0338 \mathrm{lbs} / \mathrm{sec})$.

8.1.7 Retura to para. 6.2.18 of Cold Leak and Outgassing Procedure (KIPS1020305).

8.1.8 Upon completion of para. 6.2.19 of Cold Leak and Outgassing Procedure (KIPS1020305) measure the circuit resistance $\left(R_{c_{2}}\right)$ of the EHSA as in para. 5.2. 4.

8.1.9 Calculate and record the percentage difference as follows:

$$
P d=\frac{R_{c_{1}}-R_{c_{2}}}{R_{c_{1}}} \times 100
$$

where,

$$
\mathbf{R}_{\mathbf{c}_{1}}=\begin{aligned}
& \text { circuit resistance measured in para. 6.2.5 of Cold Leak and } \\
& \text { Outgassing Procedure (KIPS1020305) }
\end{aligned}
$$

Pd may not exceed $5 \%$.

8.1.10 By looking at the temperature measurement readings, verify that none of the thermocouples inside the EHSA was showing any anomalous behavior such as reverse polarity or open circuit.

8.1.11 Return to para. 6.2.2 of Cold Leak and Outgassing Procedure (KIPS1020305). 
AER 1504-10

APPENDIX I

Page 13

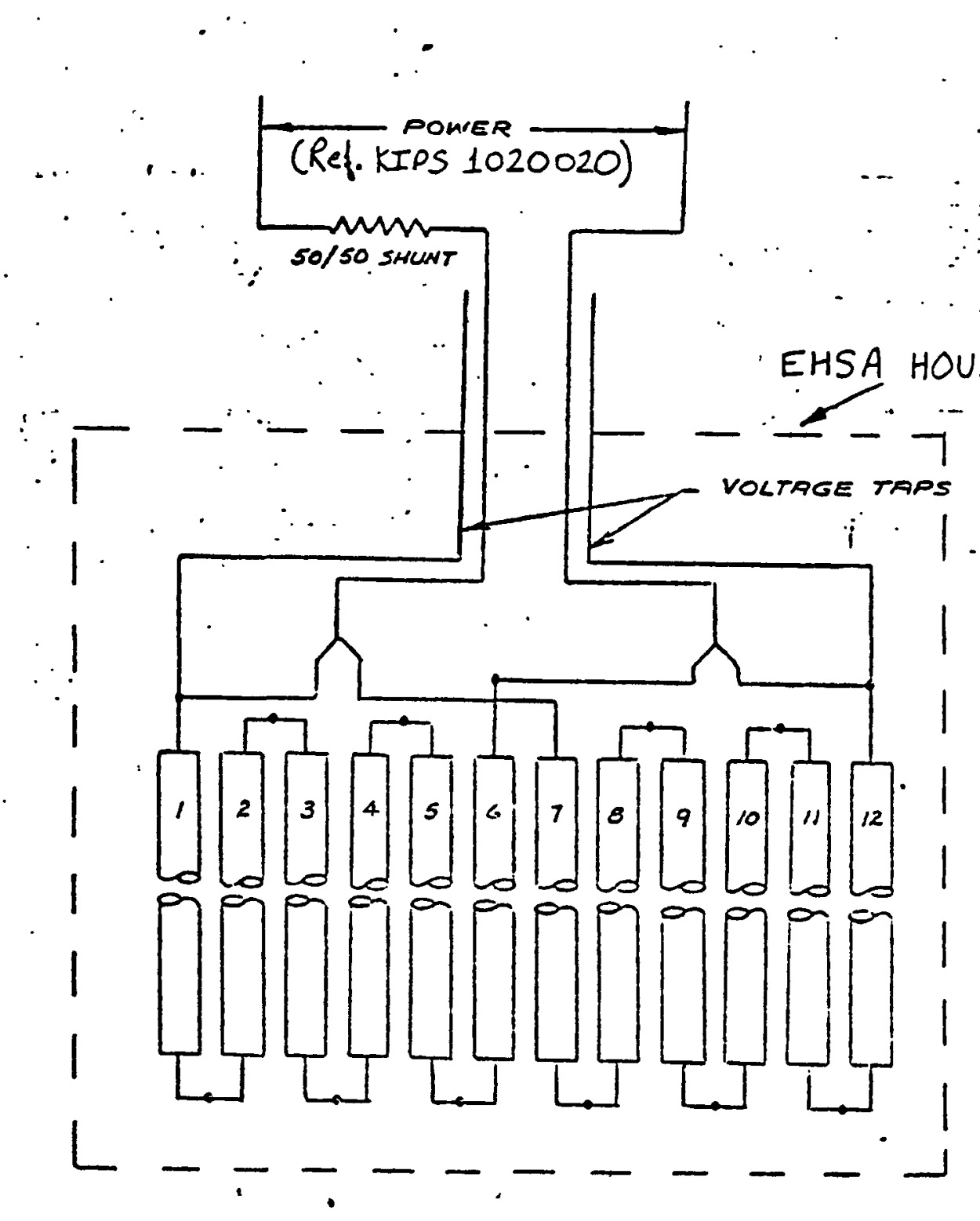

\footnotetext{
- E्वात्तs 2

HEATER WIEING SCHEMATIC
}

1 


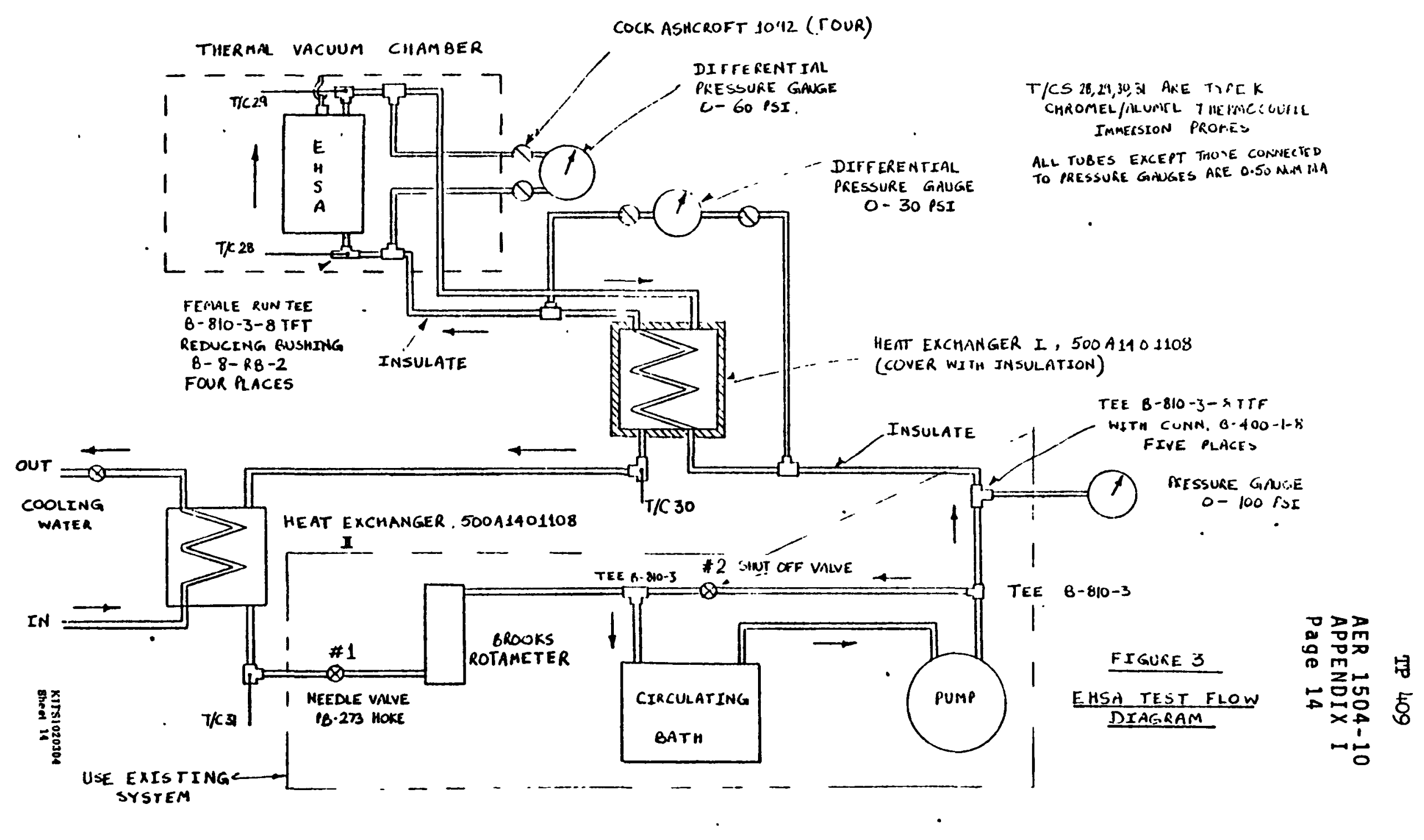


AER 1504-10

APPENDIX I

Page 15

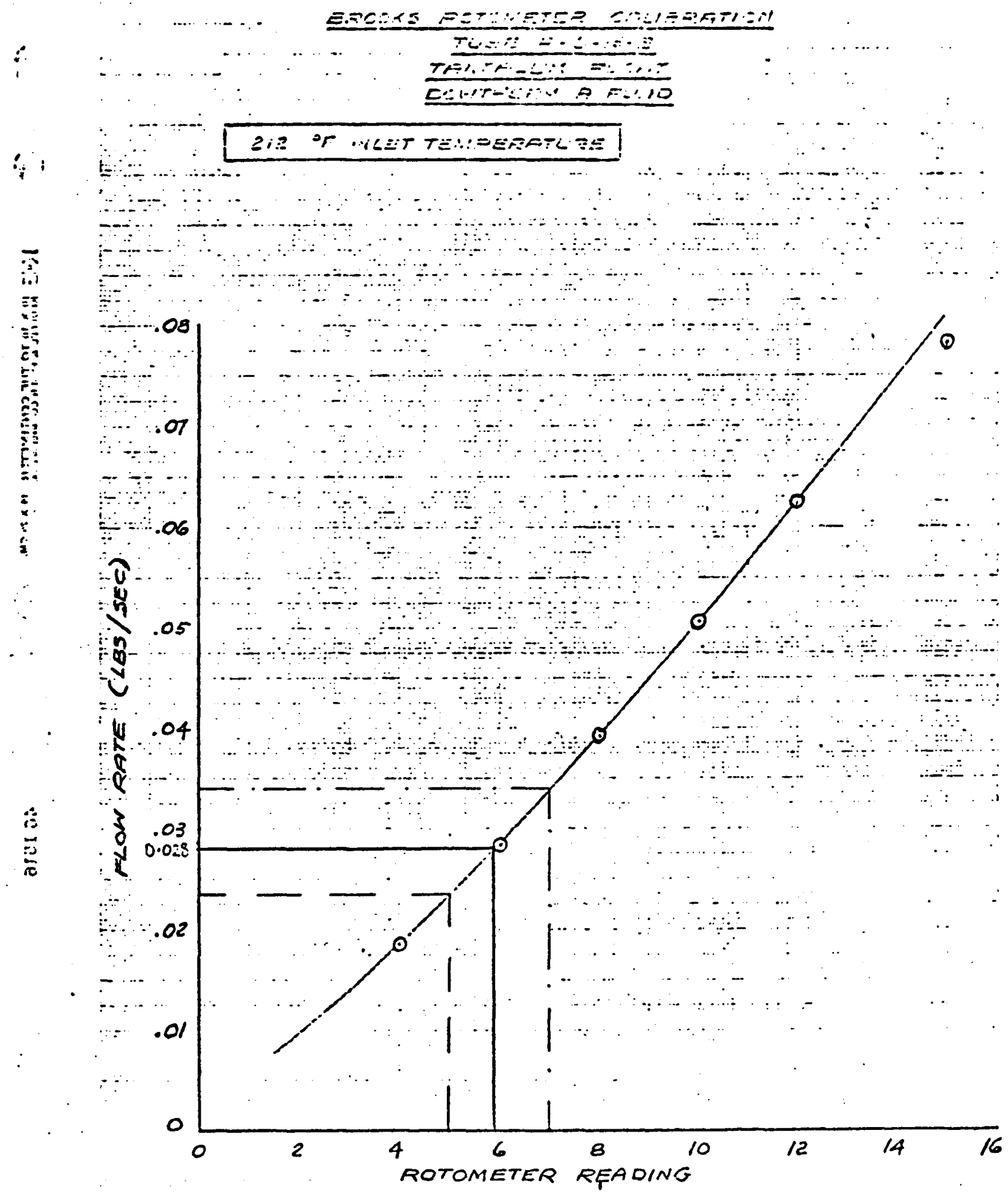

FIGURE 4. ROTAMETER CALIBRATIIN CURVE. $\because \because 7 ;$ 


\section{-}

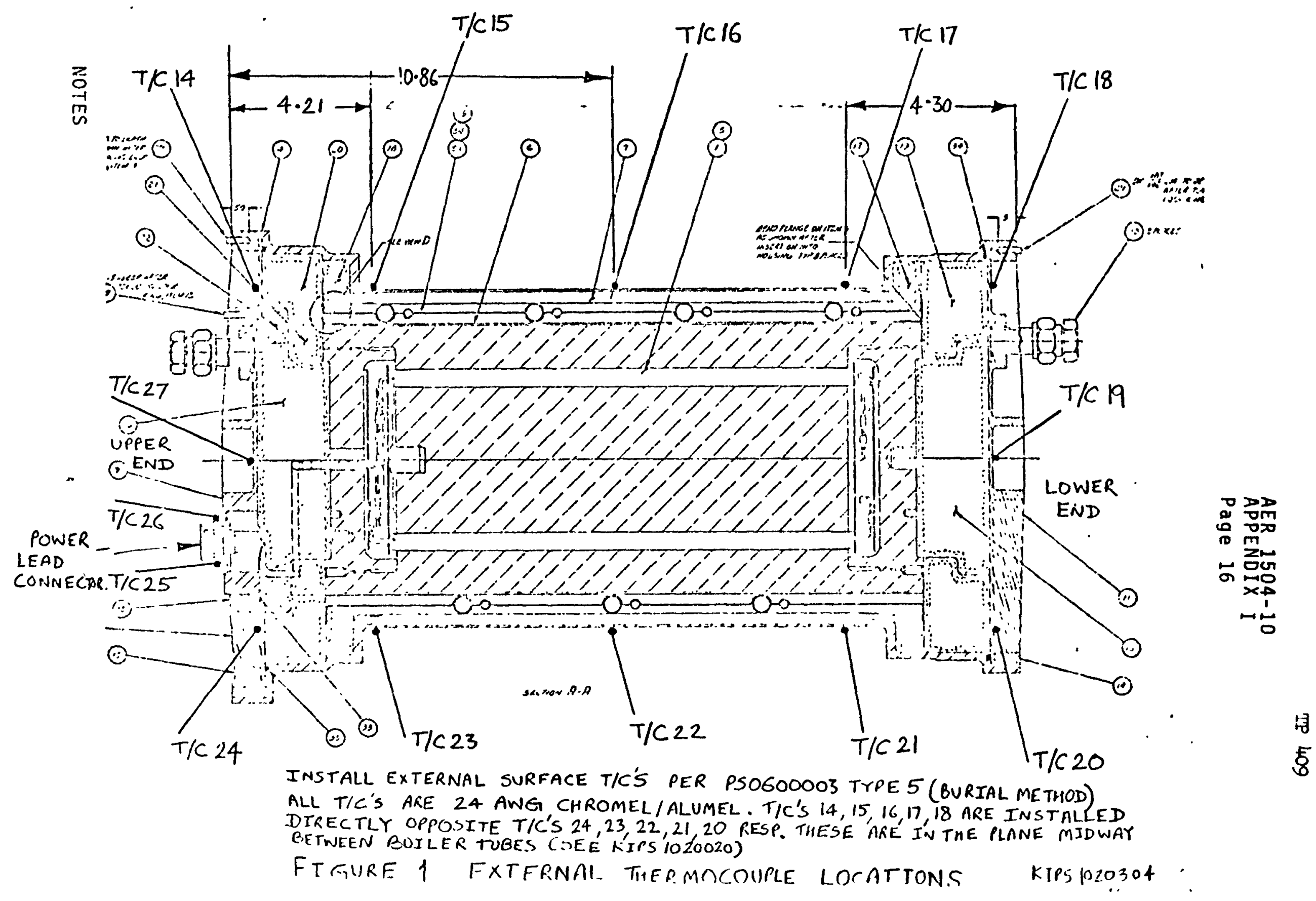




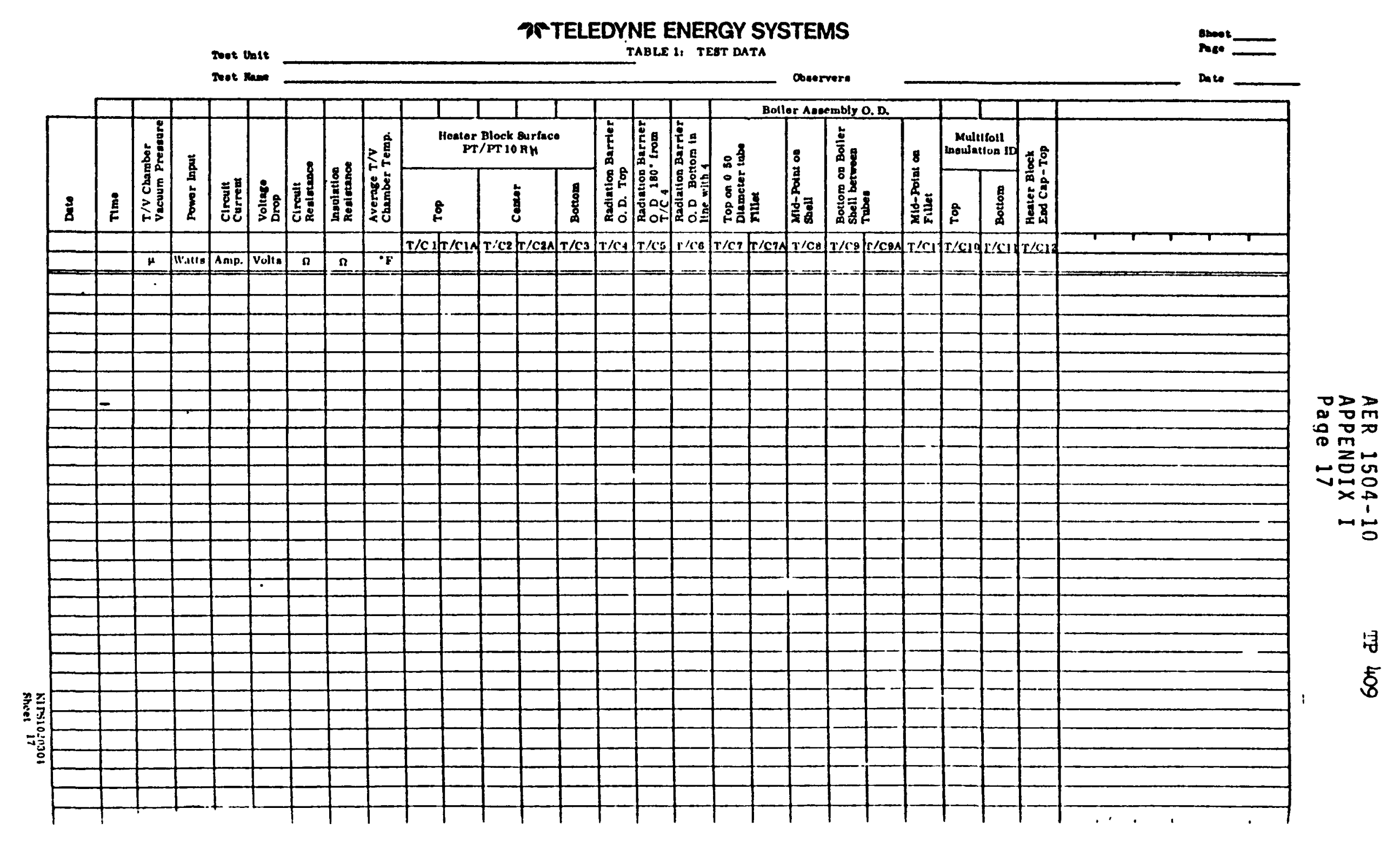




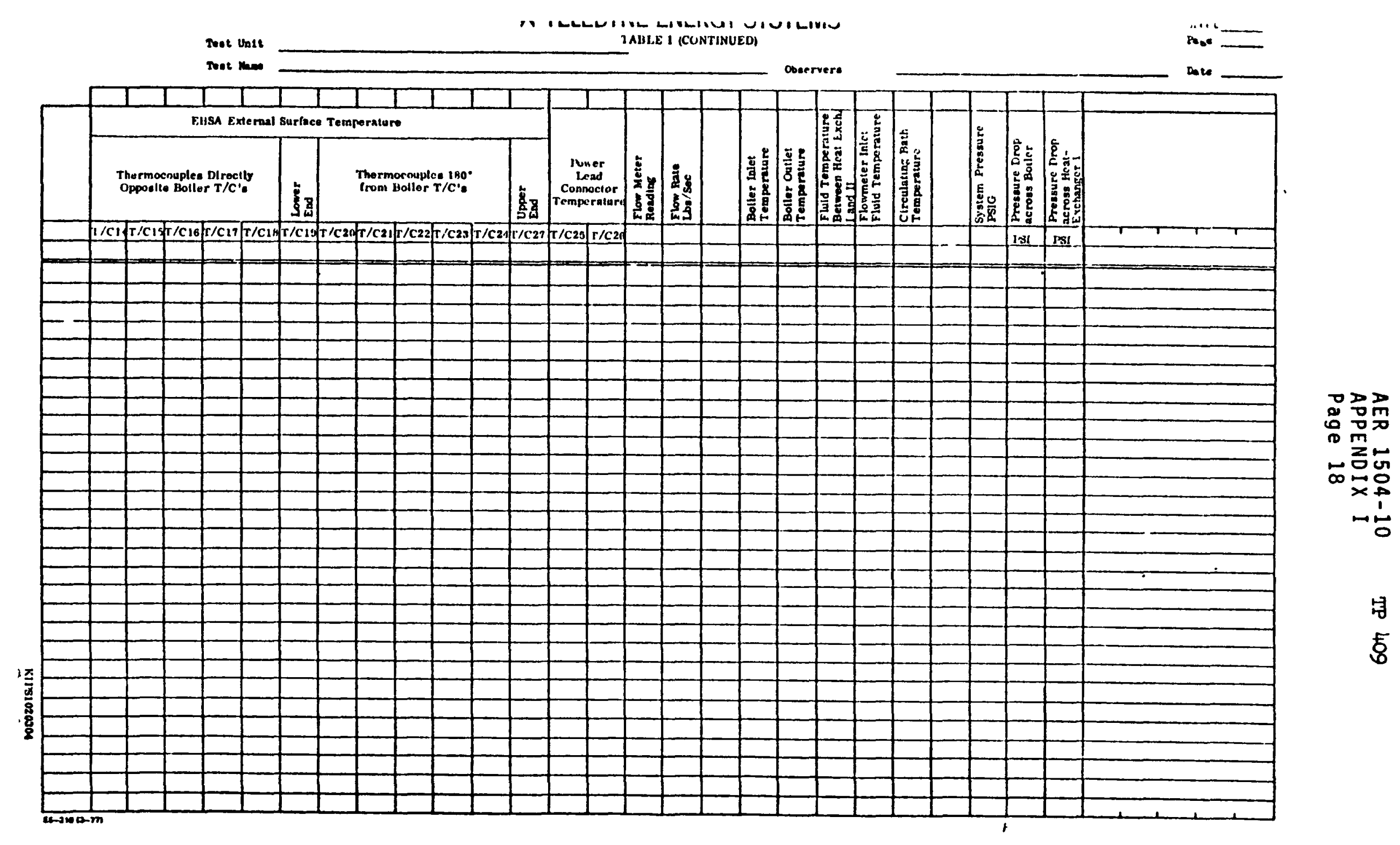


AER 1504-10

APPENDIX I

Page 19

TABLE 2

Soturofion Properties of DOWTMERM A

(English Unirs)

\begin{tabular}{|c|c|c|c|c|c|c|c|c|c|c|}
\hline \multicolumn{2}{|c|}{ remplearute } & \multicolumn{2}{|c|}{ vapor messure } & \multicolumn{3}{|c|}{ ENrmater } & $\begin{array}{l}\text { SPFCINIC } \\
\text { MEA }\end{array}$ & \multicolumn{2}{|c|}{ otmsir } & \multirow{2}{*}{ 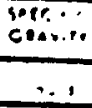 } \\
\hline \multirow[b]{2}{*}{ ४. } & \multirow[b]{2}{*}{ •c. } & Absolute & Cowal & Laved & 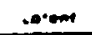 & roser & liaved & liaved & vasoor & \\
\hline & & to rin 1 & $\begin{array}{l}\text { Pecertm } \\
\text { in. Ho }\end{array}$ & \multicolumn{3}{|c|}{ sunc. } & en/libices & \multicolumn{2}{|c|}{$10 \mathrm{~m}^{3}$} & 139: \\
\hline $\begin{array}{l}52.4 \\
10 \\
70 \\
60\end{array}$ & $\begin{array}{l}120 \\
138 \\
218 \\
36.7 \\
322\end{array}$ & 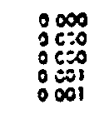 & $\begin{array}{l}307 \\
792 \\
092 \\
092 \\
092\end{array}$ & $\begin{array}{l}0: \\
2: 1 \\
: 13\end{array}$ & $\begin{array}{l}1792 \\
1794 \\
1739 \\
1720 \\
170\end{array}$ & $\begin{array}{l}1738 \\
1768 \\
1793 \\
1819 \\
1645\end{array}$ & $\begin{array}{l}5371 \\
0374 \\
0397 \\
0: 31 \\
939\end{array}$ & 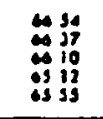 & 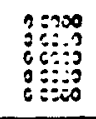 & : \\
\hline $\begin{array}{l}100 \\
110 \\
120 \\
190 \\
110\end{array}$ & $\begin{array}{l}37: 0 \\
43 j \\
404 \\
30.0\end{array}$ & $\begin{array}{l}0 \text { cot } \\
0 \% 2 \\
0 \quad c=3 \\
0005 \\
0 . \infty 1\end{array}$ & $\begin{array}{l}3992 \\
2992 \\
7992 \\
8992 \\
30.91\end{array}$ & $\begin{array}{l}176 \\
21 \\
215 \\
03 \\
\vdots 3 \\
3 \\
3\end{array}$ & $\begin{array}{l}1090 \\
1084 \\
1078 \\
1060 \\
1008\end{array}$ & $\begin{array}{l}187 ? \\
1829 \\
1027 \\
1059 \\
1924\end{array}$ & $\begin{array}{l}0.388 \\
0.382 \\
0390 \\
0.000 \\
0.03\end{array}$ & 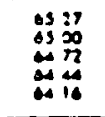 & 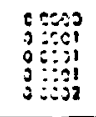 & : \\
\hline $\begin{array}{l}190 \\
160 \\
170 \\
180 \\
180\end{array}$ & $\begin{array}{l}15: 0 \\
11: \\
78.7 \\
172\end{array}$ & 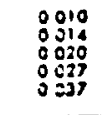 & 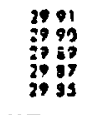 & $\begin{array}{l}37.9 \\
41.8 \\
49.7 \\
34.1\end{array}$ & $\begin{array}{l}1634 \\
1627 \\
1918 \\
1604 \\
1504\end{array}$ & $\begin{array}{l}2013 \\
301 \\
3015 \\
310 j \\
213.5\end{array}$ & $\begin{array}{l}0497 \\
0411 \\
0411 \\
0411 \\
0.422\end{array}$ & $\begin{array}{l}3318 \\
63 \text { s0 } \\
03.32 \\
6303 \\
0213\end{array}$ & 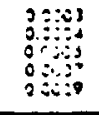 & $\begin{array}{c}1: 3 \\
\vdots \\
\vdots \\
\vdots \\
\vdots \\
\vdots \\
\vdots \\
\vdots\end{array}$ \\
\hline $\begin{array}{l}300 \\
310 \\
720 \\
730 \\
740\end{array}$ & $\begin{array}{l}139 \\
019 \\
104.4 \\
110.0 \\
119 .\end{array}$ & $\begin{array}{l}0.051 \\
0.601 \\
0.691 \\
0.120 \\
0.10\end{array}$ & 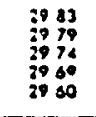 & $\begin{array}{l}28.2 \\
\$ 2.8 \\
\$ 4.9 \\
71.3 \\
75.7\end{array}$ & $\begin{array}{l}191.3 \\
1972 \\
1943 \\
1951 \\
194.0\end{array}$ & $\begin{array}{l}2108 \\
2108 \\
2231 \\
2264 \\
2075\end{array}$ & $\begin{array}{l}0426 \\
0428 \\
0433 \\
0437 \\
0.440\end{array}$ & 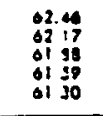 & $\begin{array}{l}0 \\
0 \\
0\end{array}$ & 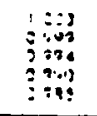 \\
\hline $\begin{array}{l}290 \\
760 \\
270 \\
290 \\
790\end{array}$ & $\begin{array}{l}121.1 \\
1298 \\
1327 \\
137.8 \\
143\end{array}$ & $\begin{array}{l}0.20 \\
0.35 \\
0.32 \\
0.41 \\
0.51\end{array}$ & $\begin{array}{l}5952 \\
7900 \\
2926 \\
7969 \\
3159\end{array}$ & $\begin{array}{l}80.1 \\
84.5 \\
890 \\
93.6 \\
98.1\end{array}$ & $\begin{array}{l}1520 \\
1520 \\
1510 \\
\text { is9.8 } \\
\text { ist. }\end{array}$ & $\begin{array}{l}223.1 \\
230.5 \\
240.9 \\
245.5 \\
247.0\end{array}$ & $\begin{array}{l}0.446 \\
0.48 \\
0.451 \\
0.45 \\
0.450\end{array}$ & $\begin{array}{l}01: 0 \\
00.11 \\
60.41 \\
6.11 \\
90.11\end{array}$ & 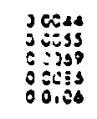 & 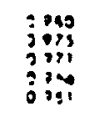 \\
\hline $\begin{array}{l}390 \\
310 \\
320 \\
330 \\
340\end{array}$ & $\begin{array}{l}148.9 \\
194 . \\
1600 \\
105.4 \\
171.1\end{array}$ & $\begin{array}{l}0.63 \\
678 \\
0.96 \\
1.17 \\
1.41\end{array}$ & $\begin{array}{l}37.65 \\
39 \\
37.97 \\
3795 \\
2706\end{array}$ & $\begin{array}{l}102.7 \\
1074 \\
112.1 \\
116.1 \\
121.5\end{array}$ & $\begin{array}{l}147: \\
149 \\
145: \\
1458\end{array}$ & $\begin{array}{l}250 \% \\
754: 2 \\
257 \% \\
361: 0 \\
263.3\end{array}$ & $\begin{array}{l}0.469 \\
0.484 \\
0.470 \\
0.474 \\
0.477\end{array}$ & $\begin{array}{l}9950 \\
9930 \\
9199 \\
9139 \\
99.20\end{array}$ & 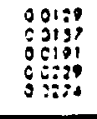 & 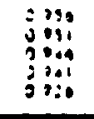 \\
\hline $\begin{array}{l}390 \\
390 \\
370 \\
390 \\
390\end{array}$ & $\begin{array}{l}176.7 \\
162.2 \\
1078 \\
103.3 \\
198.8\end{array}$ & $\begin{array}{l}170 \\
2.03 \\
3.42 \\
3.86 \\
3.37\end{array}$ & $\begin{array}{l}30.47 \\
25.80 \\
25 \$ 0 \\
24 . i 1 \\
23.07\end{array}$ & $\begin{array}{l}123: 3 \\
131.2 \\
1300 \\
1408 \\
1459\end{array}$ & $\begin{array}{l}142.9 \\
1419 \\
1408 \\
1398 \\
198.7\end{array}$ & $\begin{array}{l}289.1 \\
279: 9 \\
276.8 \\
2807 \\
2840\end{array}$ & $\begin{array}{l}04 \$ 1 \\
0.419 \\
0.098 \\
0.492 \\
0.496\end{array}$ & $\begin{array}{l}9790 \\
3795 \\
9793 \\
9792 \\
94.70\end{array}$ & 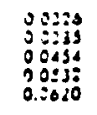 & מיו" \\
\hline $\begin{array}{l}400 \\
410 \\
420 \\
400\end{array}$ & $\begin{array}{l}264: \\
2100 \\
215.4 \\
221.1 \\
226.7\end{array}$ & $\begin{array}{l}396 \\
4.62 \\
9.37 \\
622 \\
7.18\end{array}$ & $\begin{array}{l}21.97 \\
20.92 \\
1000 \\
1727 \\
13.31\end{array}$ & $\begin{array}{l}150.9 \\
1559 \\
100.9 \\
1600 \\
171 \text { i }\end{array}$ & $\begin{array}{l}1378 \\
136.8 \\
1758 \\
1245 \\
133.5\end{array}$ & $\begin{array}{l}393.5 \\
392.5 \\
396.5 \\
320.5 \\
324.6\end{array}$ & $\begin{array}{l}0.550 \\
0.903 \\
0.907 \\
0.511 \\
0.514\end{array}$ & $\begin{array}{l}5037 \\
3005 \\
3532 \\
9530 \\
3530\end{array}$ & 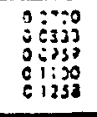 & 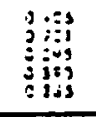 \\
\hline $\begin{array}{l}40 \\
490 \\
480 \\
490 \\
490\end{array}$ & $\begin{array}{l}232.2 \\
737 \\
24.3 \\
24.3 \\
234.4\end{array}$ & $\begin{array}{r}1.23 \\
10.77 \\
12.24 \\
13.24\end{array}$ & $\begin{array}{r}13.13 \\
10.71 \\
5.00 \\
3.01 \\
1.31\end{array}$ & $\begin{array}{l}174.3 \\
1815 \\
190.7 \\
192.0 \\
197.3\end{array}$ & $\begin{array}{l}1321 \\
1310 \\
150.3 \\
129 \\
1: 80\end{array}$ & $\begin{array}{l}305.4 \\
3124 \\
317.5 \\
321.1 \\
325.3\end{array}$ & $\begin{array}{l}0.511 \\
0.572 \\
0.326 \\
0.529 \\
0.333\end{array}$ & $\begin{array}{l}9472 \\
9438 \\
9404 \\
3370 \\
33.35\end{array}$ & 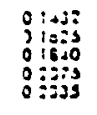 & $\begin{array}{c}3 \\
\vdots \\
\vdots \\
\vdots \vdots 1 \\
\vdots \vdots 1 \\
\vdots \vdots \\
\vdots\end{array}$ \\
\hline 494.8 & 237.1 & 14.70 & 0.00 & 199.9 & 127.4 & 927.3 & 0.513 & 53.10 & 0.2470 & os: \\
\hline $\begin{array}{l}100 \\
510 \\
520 \\
520 \\
510\end{array}$ & $\begin{array}{l}260.0 \\
365 . \\
271.7 \\
2787 \\
212.2\end{array}$ & $\begin{array}{l}13.83 \\
17.02 \\
10.79 \\
22.14 \\
24.71\end{array}$ & $\begin{array}{c}0.93 \\
2.97 \\
3 \text { of } \\
71.4 \\
10.01\end{array}$ & $\begin{array}{l}202.7 \\
305.1 \\
213.5 \\
218.0 \\
224.5\end{array}$ & $\begin{array}{l}125.9 \\
1257 \\
1245 \\
123.3 \\
130.1\end{array}$ & 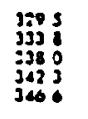 & $\begin{array}{l}0.537 \\
0.541 \\
0.545 \\
0.519 \\
0.554\end{array}$ & $\begin{array}{l}3500 \\
32 \text { o5 } \\
32.98 \\
91.93 \\
5196\end{array}$ & 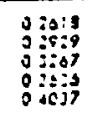 & 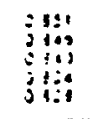 \\
\hline $\begin{array}{l}\mathbf{5 8 0} \\
\mathbf{3 8 0} \\
\mathbf{3 9 0} \\
\mathbf{5 0 0} \\
\mathbf{5 0 0}\end{array}$ & $\begin{array}{l}217: \\
2935 \\
3815 \\
304.4 \\
310.0\end{array}$ & 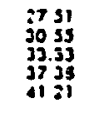 & $\begin{array}{l}1231 \\
1985 \\
11913 \\
2289 \\
2831\end{array}$ & $\begin{array}{l}230 \\
2357 \\
201 \\
241 \\
391 \\
32.8\end{array}$ & $\begin{array}{l}1 \% 03 \\
1195 \\
1182 \\
116.8 \\
\text { i1s.3 }\end{array}$ & 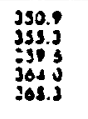 & $\begin{array}{l}0.558 \\
0567 \\
0: 67 \\
0571 \\
0575\end{array}$ & 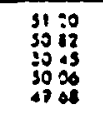 & 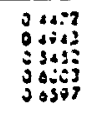 & $\begin{array}{l}3 \\
j \\
\vdots \\
\vdots \\
\vdots \\
\vdots\end{array}$ \\
\hline $\begin{array}{l}100 \\
10 \\
10 \\
10 \\
40\end{array}$ & $\begin{array}{l}3150 \\
3211 \\
325 ? \\
332: 2 \\
337.8\end{array}$ & 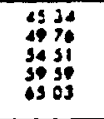 & 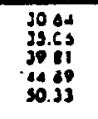 & $\begin{array}{l}2550 \\
3840 \\
790: \\
3181 \\
3020\end{array}$ & $\begin{array}{l}114 ! \\
1125 \\
1113 \\
100 \\
1003\end{array}$ & $\begin{array}{l}372.7 \\
377: \\
381: 5 \\
395 \\
3904\end{array}$ & $\begin{array}{l}0.978 \\
0.967 \\
0: 585 \\
0530 \\
0.513\end{array}$ & 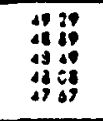 & 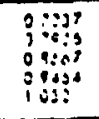 & $\begin{array}{c}\vdots \\
\vdots \\
\vdots \vdots \\
\vdots \\
\vdots \\
\vdots\end{array}$ \\
\hline $\begin{array}{l}190 \\
40 \\
400 \\
480 \\
400\end{array}$ & $\begin{array}{l}3439 \\
3480 \\
394.4 \\
3450 \\
3450\end{array}$ & 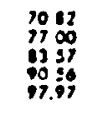 & 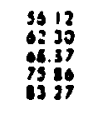 & $\begin{array}{l}2910 \\
2900 \\
3950 \\
300 \\
3122\end{array}$ & $\begin{array}{l}160: \\
105 ? \\
103: \\
1050 \\
1003\end{array}$ & $\begin{array}{l}394 \\
320 \\
203 \\
403 \\
415 \\
412.5\end{array}$ & 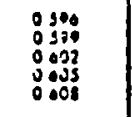 & $\begin{array}{l}4735 \\
46 \\
4838 \\
4534 \\
45\end{array}$ & 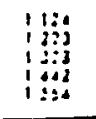 & $\begin{array}{l}: \because " \\
\vdots \because \vdots \\
\vdots \\
\vdots\end{array}$ \\
\hline $\begin{array}{l}700 \\
710 \\
730 \\
7100 \\
710\end{array}$ & $\begin{array}{l}371: \\
370.7 \\
3929 \\
319 \\
373.3\end{array}$ & $\begin{array}{l}105: \\
1148 \\
1235 \\
1323 \\
1451\end{array}$ & $\begin{array}{r}1110 \\
1095 \\
10350 \\
11 ; 50 \\
1: 7\end{array}$ & $\begin{array}{l}3113 \\
3: 15 \\
393 \\
3310 \\
3434\end{array}$ & $\begin{array}{l}99 \\
96 \\
93 j \\
03 \\
131 \\
11.2\end{array}$ & $\begin{array}{l}4159 \\
471.3 \\
1239 \\
435 ? \\
4140\end{array}$ & $\begin{array}{lll}0 & 611 \\
0 & \$ 11 \\
0 & 019 \\
0 & 8 \% 3 \\
0 & 0.33\end{array}$ & $\begin{array}{l}4503 \\
4490 \\
4193 \\
4 j 90 \\
43: 0\end{array}$ & $\begin{array}{cc}1 & 0 \\
1 & 1 \\
1 & 0 \\
1 & \vdots \\
\vdots & \vdots \\
2 & \vdots\end{array}$ & $\begin{array}{c}\vdots: \vdots \\
\vdots \because: \\
\vdots \because \because\end{array}$ \\
\hline $\begin{array}{l}790 \\
780 \\
770 \\
790 \\
790\end{array}$ & $\begin{array}{l}3019 \\
104: 8 \\
1100 \\
4196 \\
121.1\end{array}$ & 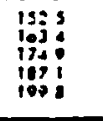 & $\begin{array}{l}13750 \\
1.390 \\
10020 \\
1: 140 \\
199.10\end{array}$ & 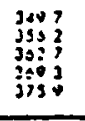 & $\begin{array}{l}38: 2 \\
81 \\
512 \\
32 \\
30 \\
1\end{array}$ & $\begin{array}{l}4359 \\
1435 \\
1470 \\
4515 \\
450\end{array}$ & 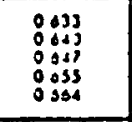 & 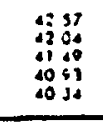 & 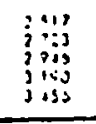 & $\begin{array}{l}\because \\
\vdots \\
\vdots\end{array}$ \\
\hline$\$ 100$ & 4807 & :13 & 18100 & 3327 & $n$ & 4002 & 0.73 & 2014 & 31,9 & $: \therefore$ \\
\hline
\end{tabular}

KIIS1020304 Sineel 1:I 
APPENDIX II

TELEDYNE ENERGY SYSTEMS

EHSA PERFORMANCE TEST ANALYSIS

NUMBER KIPS-DKD-086 


\section{TATELEDYNE ENERGY SYSTEMS}

Subject:

KIPS EHSA Performance Test Analysis

Reference:

1. KIPS-VRL-040 "Analytical Representation of Multifoil Insulation," 22 October 1976

2. KIPS-DKD/VRL-066 'KIPS Electrical Heat Source Power Conductor Thermal Analysis," 11 February 1977

3. KIPS-VRL-074 'KIPS EHSA Thermocouple Lead Thermal Analysis, 17 March 1977

\section{Summary and Conclusions}

The Electrical Heat Source Assembly (KIPS1020020-039) was tested inside a the rmal vacuum chamber, in order to evaluate its performance and to determine the heat losses. In view of the presence of large uncertainty in the measurement technique and the Intricate design of the EHSA, it became difficult to determine exactly the amount of heat losses. Basod on the various temperature distributions over the EHSA and its surroundings, the heat losses are seen to be of the order of 60 watts. However, the heat balance between the amount of heat put into the EHSA and that carried away by the boller, shows heat losses to be, of the order of 150 watts (dependent on Dowtherm flow rate). The exact amount of heat losses are expected to be somewhere in-between but, closer to 60 watts. It is noticed that in order to make a correct heat balance, the amount of electrical heat that is being dissipated inside the EHSA by the power lead wires, must be taken into consideration. It is expected that this heat dissipation Is relatively large, and of the order of 70 watts.

The temperature of the heater block was lower than that expected. Because of its large thermal inertia, the temperature of the heater block had not quite stabilized during the period of the test, however, the change in the healer block temperature was extremely gradual and it was not noticeable during the test. The electrical realstance of the healer block was seen to be rising gradually, thus requi ring constant readjustment of the power input throughout the test. As a consequence of the lower heater block temperature, the EISA was draiving larger clrcuit current. Most of the components of the EIISA were seen to be at their nominal temperatures. The internal temperature of the electrical power lead connector is calculated to be as high as $560^{\circ} \mathrm{F}$. In reality, the inside temperature of the connector is not expected to be this high, nevertheless it seems to be high enough to require close scrutiny. 
The error in the thermocouple temperature measurement due to spurious emf generated at the connector was, at the nominal operating temperaturcs, found to be less than $50^{\circ} \mathrm{F}$ in the case of heater block and less than $25^{\circ} \mathrm{F}$ in the case of boller. At both places the thermocouples were seen to be reading less than the actual temperatures.

\section{Implications}

Based on the evaluation of these test data, it is the author's contention that the following steps should be taken.

1. The heater block seems to take considerably longer time to reach steady thermal state. This should be brought to the attention of GDS test personnel.

2. There is a substantially large amount of heat that is being dissipated by the electrical lead wires inside the EHSA, which is not recorded on the wattmeter. In order to make proper heat balance, this additional power dissipation must be taken into consideration. It is suggested that the exact amount of this heat dissipation be determined in one of the units by placing voltage taps as close to the connector as possible.

3. The housing power lead connector is at a rather high temperature. It is suggested that we should use high temperature solder ( $95 \%$ lead $-5 \%$ tin) in this reglon

4. In order to completely and conclusively evaluate the performance of the EHSA, It is suggested that one of the units should be subjected to more extensive tests. Such tests would determine the effect of various parameters on the EHSA performance. In order to accomplish this, the present test setup should be modified to achieve higher flow measurement accuracy and higher temperatures and preferably use a EHSA boiler with the insert in it.

5. The lower temperature of the heater block may be due to higher than the expected emissivity of the radiation barrier. It is suggested that the emissivity of a representative sample of the radiation barrier be measured in order to verify this.

\section{Discussion}

The Electrical Heat Source Assembly (KIPS1020020-039) was tested inside a thermal vacuum chamber in order to evaluate its performance and to determine the heat losses. The EHSA was connected to the Dowtherm now loop as shown in Figure 1. The position of the thermocouples attached external to the EHSA is shown in Figure 2. The thermal vacuum chamber was evacuated to less than $1 \mu$. The test was performed for three different now rates. An attempt was made to keep the temperature of the fluid entering the boiler constant at three different values. Unfortunately, due to limitations imposed by the test setup, it becane difficult to control the temperature exactly. Moreover, it has not been possible to exceed $425^{\circ} \mathrm{F}$ at the boiler inlet.

The heat lost from the EHSA is that part of the total heat input to the EHSA, which is not carried away by the boiler due to change in internal energy of the fluid flowing 


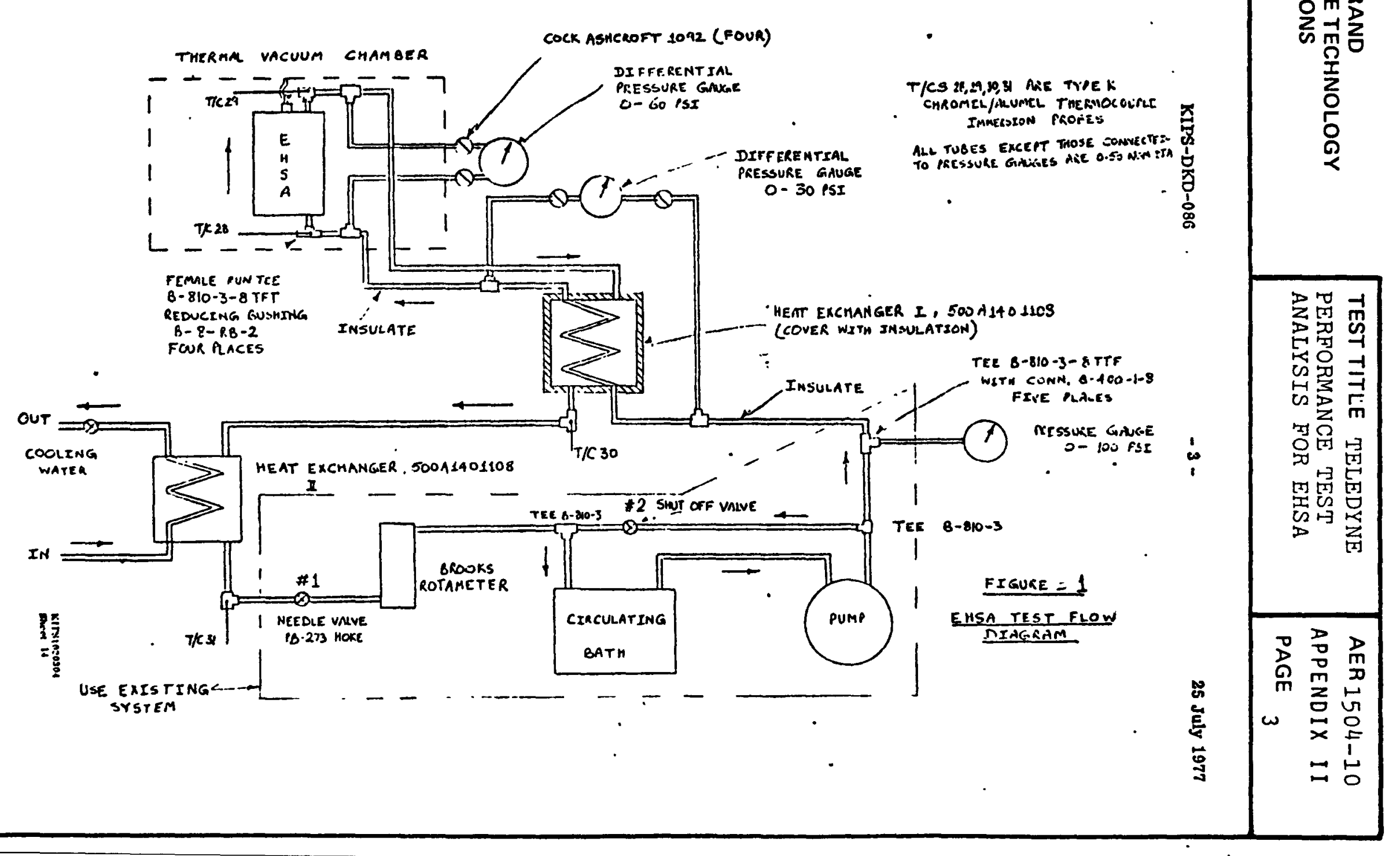




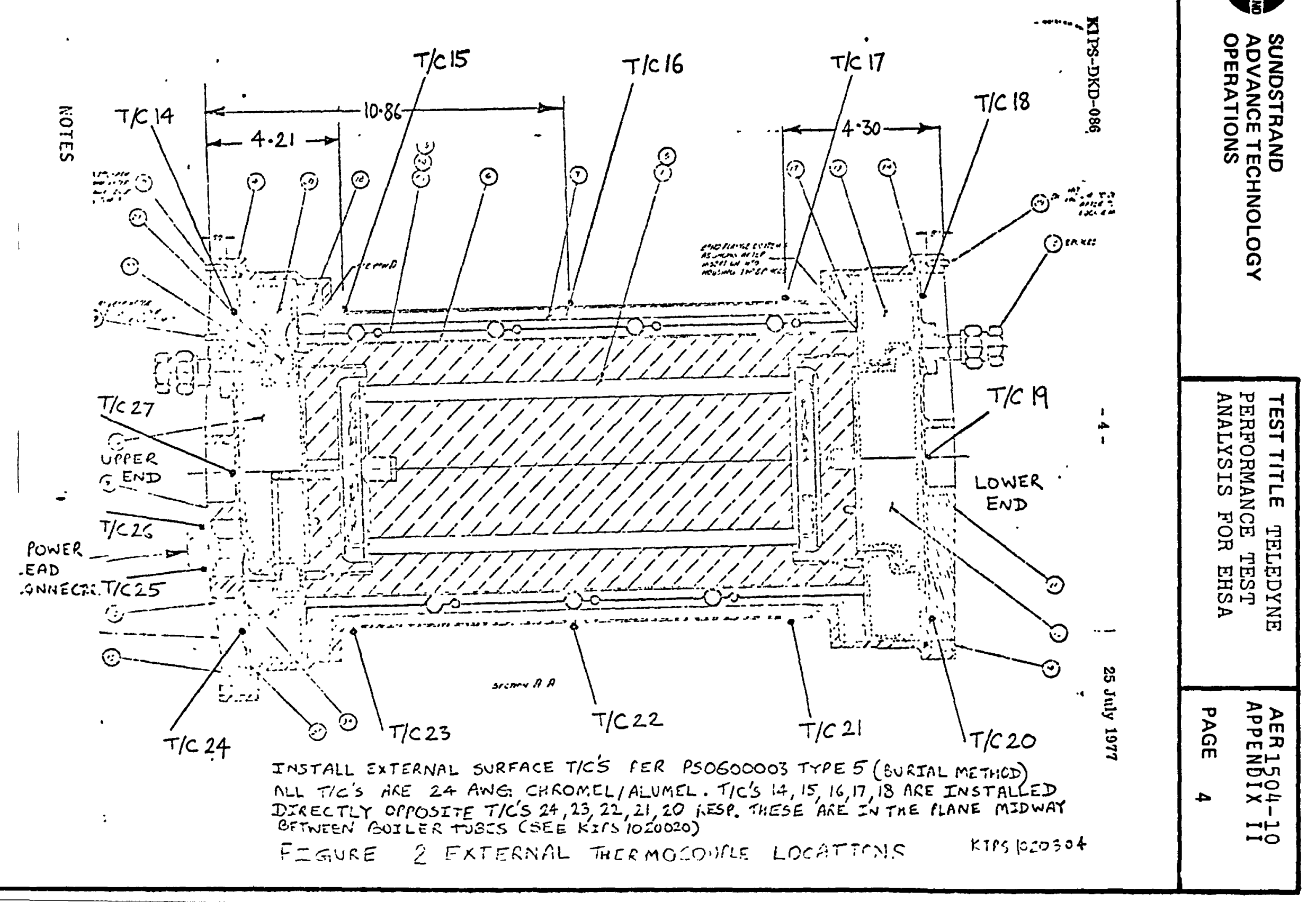


through it. One way of determining the heat losses is to find the difference in the amount of power that is supplied to the unit and the amount that is carried away by the boiler fluid. The second way, which is more involved, is to calculate the heat losses directly by knowing the temperature of the various components of the EISA and its surroundings. Because of its inherent simplicity, the heat losses determined by the first method should give better indication of its magnitude. Nevertheless, it is shown . In Appendix $A$ that the determination of heat losses by this method is strongly dependent on the accuracy of the flow measurement. In particular it is seen that the uncertainty in the measurement of heat removed by the boiler could be as large as $8 \%$, whereas the amount of heat loss from the EHSA is not expected to exceed 5\% of the total power input.

The results of the test are summarized in Tables 1 and 2. It is found that the thermocouples, immersed in flow lines to measure the boller inlet and outlet temperatures, would indicate erroneous readings unless they are placed close to the EHSA. In view of this, two thermocouples were attached to the boiler, one at inlet and the other at outlet each approximately 3 " from the ends of the EHSA. In the absence of the heat transfer from the surface of the tubes which were well insulated and placed in vacuum, these thermocouples are considered to give more accurate boiler inlet and outlet temperatures and consequently a more accurate value of the heat carried away by the boller. Table 1 contains the data based on the boiler inlet and outlet temperatures measured by immersed thermocouples and Table 2 contains the data based on T/C's attached to boiler inlet and outlet tubes. In either case the trend of the results remain the same.

For a constant flow rate the effect of boiler inlet temperature on the temperature drop across the boiler seems to be negligible. In view of this we can plot graphs in Figures 3 and 4 . In Figure 3 are plotted the heat carried away by the boiler and the temperature difference across the boiler vs the flow rate. In Figure 4 are plotted the heat carried away by the boiler and the temperature difference across the boiler vs the boller tube Reynolds number. It is seen that, whereas, in the first case the temperature drop is well correlated with the flow rate; it is the heat carried away by the boller that is well correlated with the Reynolds number in the second case. The temperature drop indicates exponential decay with the increase in flow rate which would be expected since the total amount of heat transferred by the boiler is a finite quantity. similarly the heat carried away by the boiler indicates an exponential increase with the Reynolds number. It is interesting to note that the maximum amount of heat carried away by the boiler does not increase significantly after a certain Reynolds number and the temperature drop across the boiler does not diminish significantly after a certain flow rate. This suggests that there is a limit to the amount of heat that can be removed by the boiler with the increase in the Reynolds number. Increase In the boiler tube Reynold's number is also believed to be associated with improvement 


\section{PAGE 6}

In the convective heat transfer coefficient and as a consequence the botler fin-root temperature drops and so does the boiler fin temperature. It is observed that the temperature difference betiveen the boiler fin and the fin-root is independent of the now rate and remains constant at $50 \pm 4^{\circ} \mathrm{F}$, over the range of flow considered in the teat.

It is seen that there is no large temperature variation along the length of the multifoll insulation and that its average temperature is higher than that of the boiler. It indicates that there is a local back-flow of heat from the multifoil insulation to the boiler where the boiler is relatively cooler due to the presence of colls. This seems to be quite possible in view of the fact that the multilayer insulations typically have thermal conductivities parallel to the radiation shields, that are much higher than those perpenducular to the shields. 


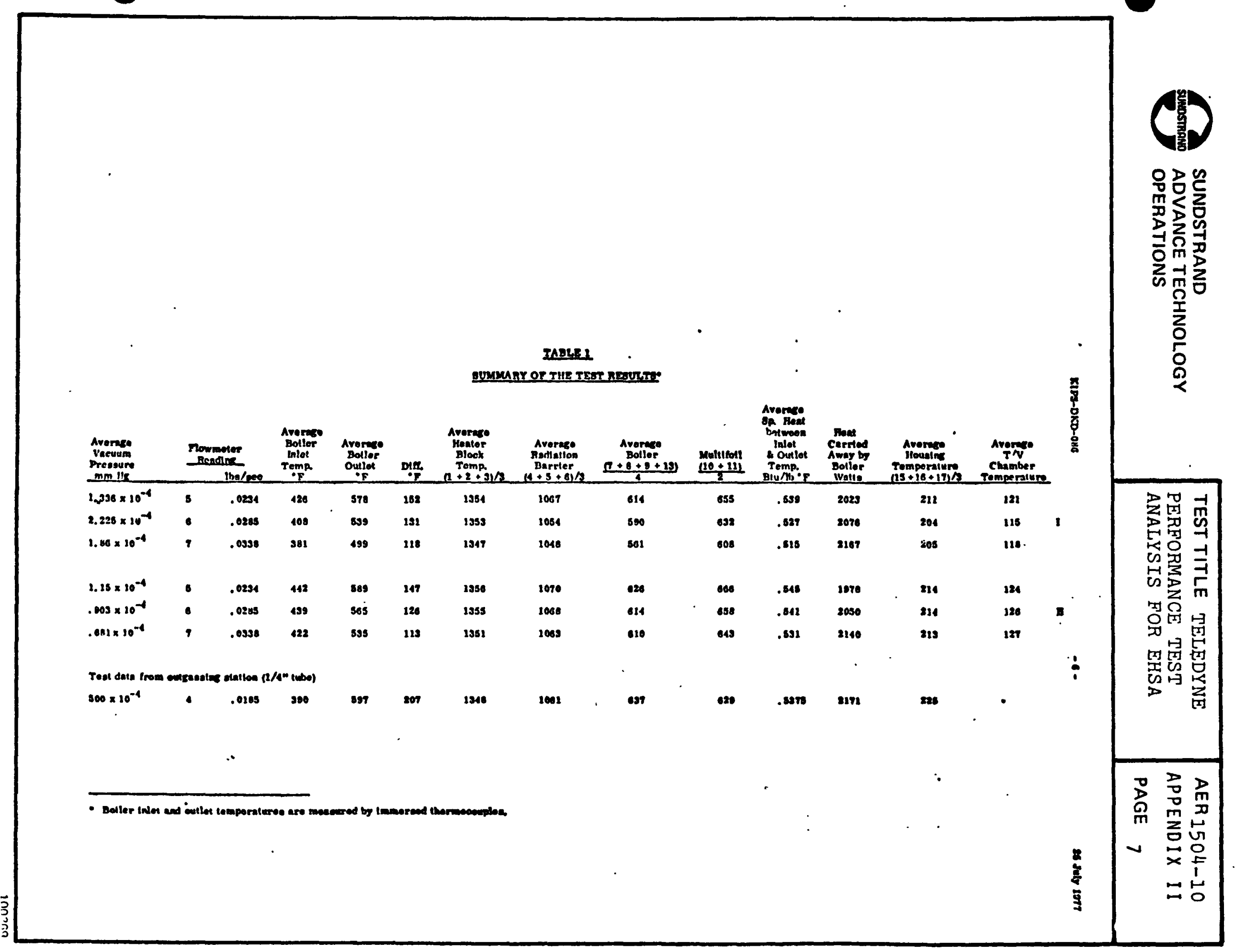


TEST TITLE TELEDYNE

PERFORMANCE TEST

ANALYSIS FOR EHSA
AER $1504-10$ APPENDIX II

PAGE 8

KIPS-DKD-086

$$
-7-
$$

26 July 1977

\section{TABLE 2}

\section{DATA BASED ON T/C'S ATTACHED TO BOILER INLET AND OUTLET TUBES}

Ibs

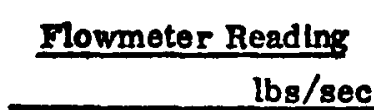

$5 \quad 0.0234$

$6 \quad 0.0285$

7

0.0338

0.0234

0.0285

0.0338
Boller Inlet Tube Temperature - $F$

412

397

371

428

427

412
Boller Outlet Tube Temperature - $F$

585

543

501

597

569

538

\begin{tabular}{ccc}
$\begin{array}{c}\text { Dlfferentlal } \\
\text { F }\end{array}$ & $\begin{array}{c}\text { Average } \\
\text { Sp. Heat } \\
\text { between } \\
\text { Inlet \& } \\
\text { Outlet } \\
\text { Btu/lb } \bullet_{F}\end{array}$ & $\begin{array}{c}\text { Heat } \\
\text { Carried } \\
\text { Away by } \\
\text { Boller } \\
\text { Watts }\end{array}$ \\
\hline 173 & .539 & 2302 \\
146 & .529 & 2322 \\
130 & .513 & 2378 \\
169 & .545 & 2274 \\
142 & .537 & 2293 \\
126 & .529 & 2377
\end{tabular}




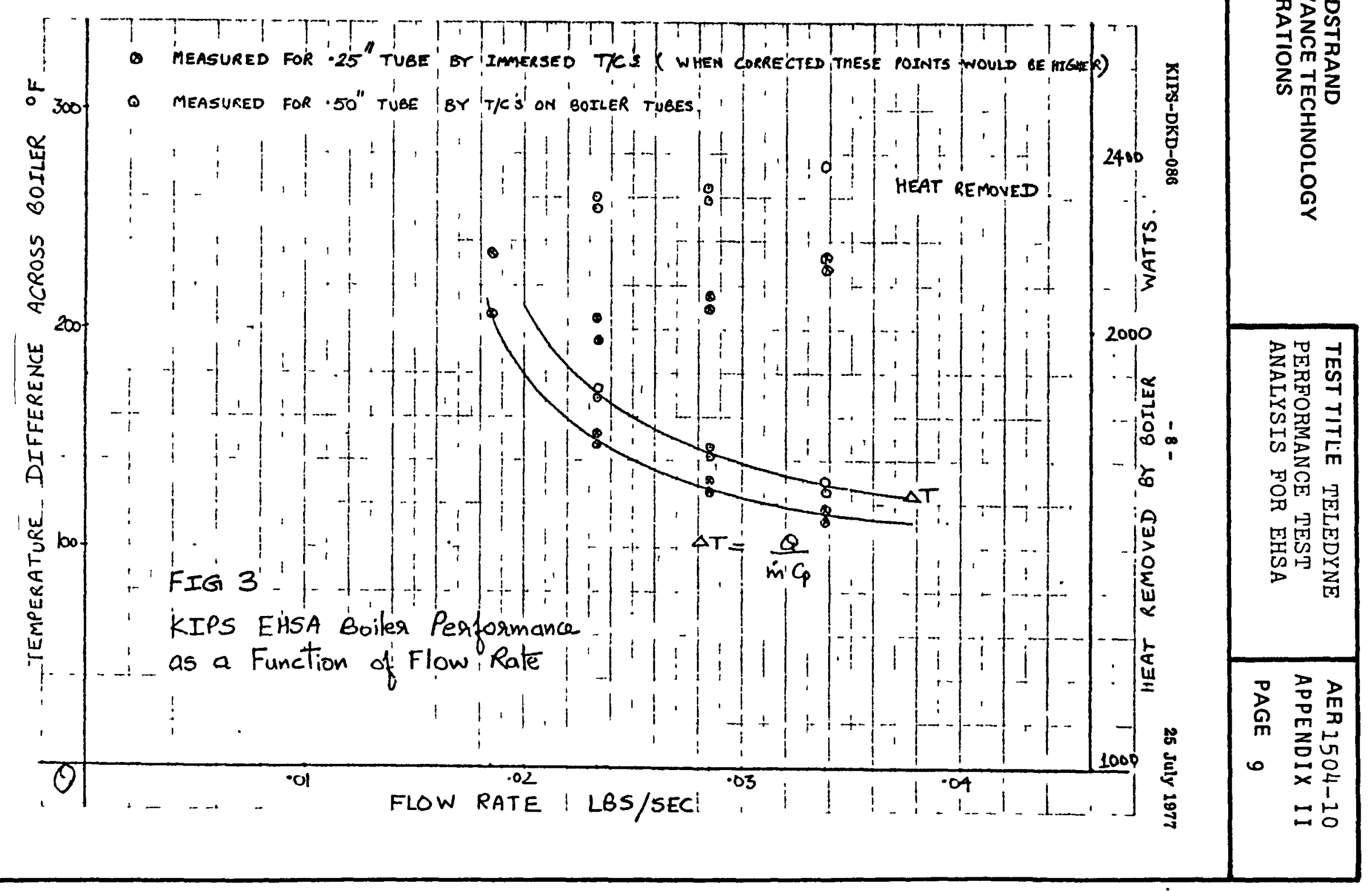




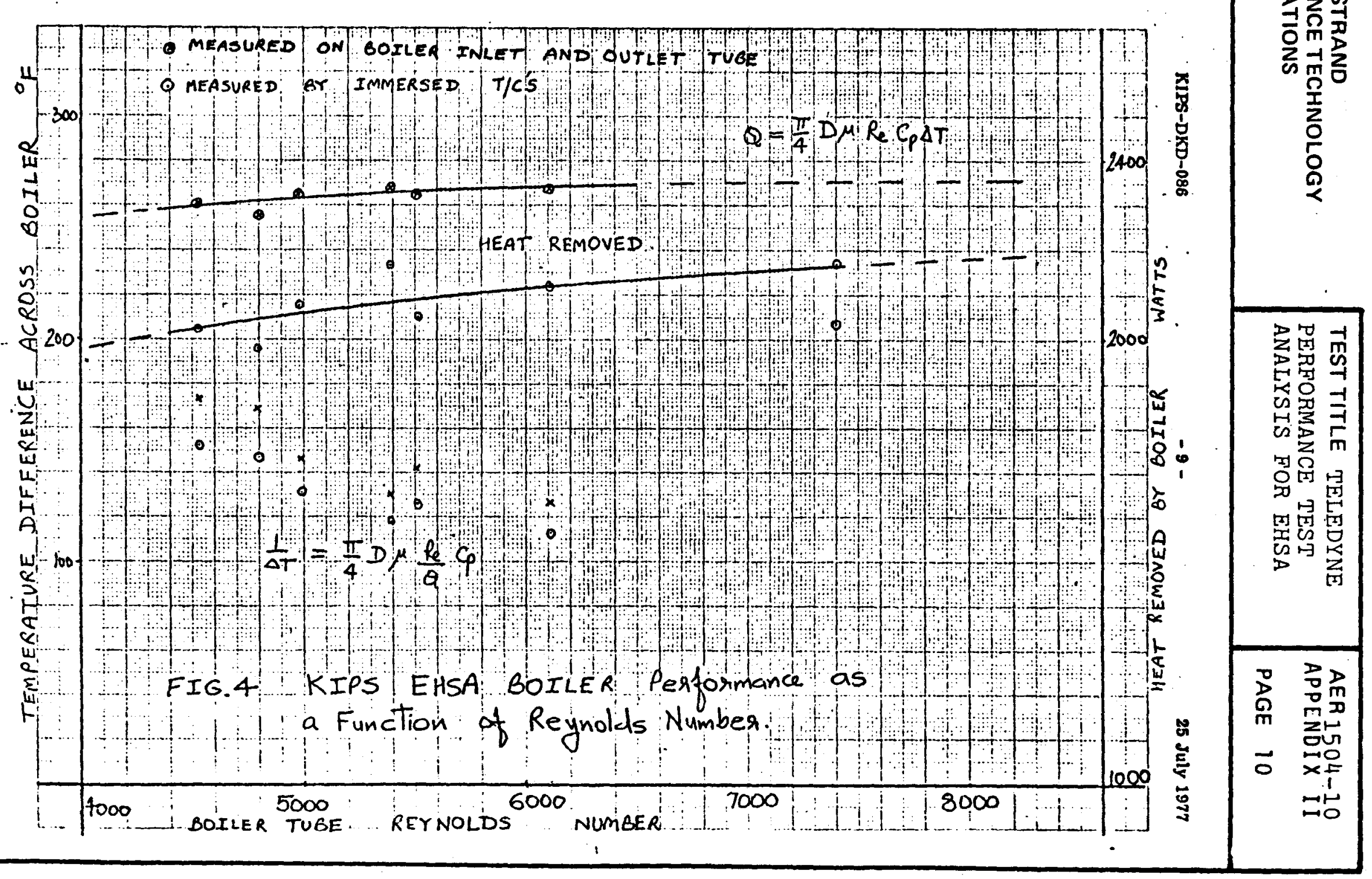


TEST TITLE TELEDYNE
AER $1504-10$ APPENDIX II

PAGE 11

For the same flow rate, a higher boller inlet temperature results in higher boiler and multifoll temperatures and higher heat losses. There is no significant effect of this on the temperature of the heater block and the radiation barrier. Thus, the effects of boller temperature much higher than those used in this test, on the heat losses must be determined. The temperatures of the heater block and the radlation barrier were found to be less than those predicted. This could be due to the emissivity of the radiation barrier being higher than that assumed or due to thermal conductive shorts between the boiler and the heater block.

The heat losses from various surfaces of the EHSA, based on their average temperatures are calculated in Appendix B. It immediately became evident that these calculations are strongly dependent on the availability of the thermal properties of these components. Nevertheless, these calculations indicate the relative hoat losses from the various surfaces of the EHSA. In Appendix $\mathrm{C}$ the heat transfer through multifoil Insulation is calculated and it is found that the effective thermal conductivity of this inculation is much higher than that reported earlier (Ref. 1).

There appears to be a temperature drop of approximately $40^{\circ} \mathrm{F}$ between the top and bottom end cover of the housing. That side of the EHSA where the power lead connector exists is at a higher temperature than the other side. This is thought to be due to the fact that additional amount of power is being dissipated on this side of the EHSA due to the presence of heater lead wires. It is found in Appendix $D$ that this amount of internal beat dissipation could be as high as 70 watts. It is further shown in Appendix $D$ that although the heat loss through the power cable is relatively small, the cable connector temperature is relatively high. Although no circumferential temperature gradients were observed on the EHSA housing, there was approximately $30^{\circ} \mathrm{F}$ drop between the diametrally opposite $\mathrm{T} / \mathrm{C}$ 's placed on the radiation barrier. This is probably because of the asymmetry introduced by the presence of spiralling boller tube. The temperature of the power lead connector was rather high at about $300^{\circ} \mathrm{F}$ on the outside shell. It could be running substantially higher on the inside near pins. 


\section{APPENDIXA \\ UNCERTAINTY IN THE HEAT TRANSFERRED BY BOILER}

Based on the single sample test data, the uncertainty in the determination of thermal energy carried away by the boiler is obtained from the following relationship.

$$
Q=m \operatorname{cp} \Delta T \times \frac{3600}{3.412} \text { watts }
$$

where, the symbols have there usual meaning. The uncertainty in the heat transfer le given by

$$
W_{Q}=\left[\left(\frac{\partial Q}{\partial \dot{m}} W_{\dot{m}}\right)^{2}+\left(\frac{\partial Q}{\partial c p} W_{c p}\right)^{2}+\left(\frac{\partial Q}{\partial \Delta T} W_{\Delta T}\right)^{2}\right]^{1 / 2} \frac{3600}{3.412}
$$

For the following typical data

$$
\begin{aligned}
& \dot{\mathrm{m}}=0.0285 \pm .0015 \\
& \mathrm{cP}=0.52 \pm 0.02 \\
& \Delta \mathrm{T}=130 \pm 6 \\
& Q=2033 \pm W_{Q}
\end{aligned}
$$

Thus the uncertainty in the measurement of heat transfer by this method turns out to be approximate y 162 watts or about $8 \%$. The largest amount of uncertainty is introduced due to our inability to measure the flow rate accurately. 


\section{APPENDIX B}

\section{CALCULATION OF HEAT LOSSES FROM THE EHSA}

Heat Loss from the Cylindrical Housing Surface

süface area $=3.305 \mathrm{ft}^{2}$

Housing surface emissivity $=0.2$

$$
-\quad Q=\frac{-E_{b_{H O}}-E_{b_{T} / V}}{\frac{1-C_{H O}}{H O}+\frac{1}{A F}}
$$

where, the symbols have their usual meaning.

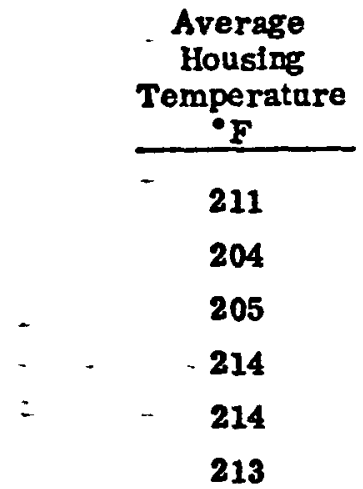

Average Temperature

\begin{tabular}{c}
$\begin{array}{c}\text { Average } T / V \\
\text { Chamber } \\
\text { Temperature }\end{array}$ \\
\hline 121 \\
115 \\
118 \\
124 \\
126 \\
127
\end{tabular}

\begin{tabular}{c}
$\begin{array}{c}\text { Heat Rejected } \\
\text { Watts }\end{array}$ \\
\hline 29.47 \\
28.25 \\
27.87 \\
29.90 \\
29.37 \\
28.69
\end{tabular}

It Is further seen from Figure B-1 that this heat rejection does not change substantially with improvement in the vacuum chamber pressure. It is not known as to how much of this heat loss is from the multifoil insulation, as the temperatures of the housing are influenced by conduction of heat along its length from the top. 


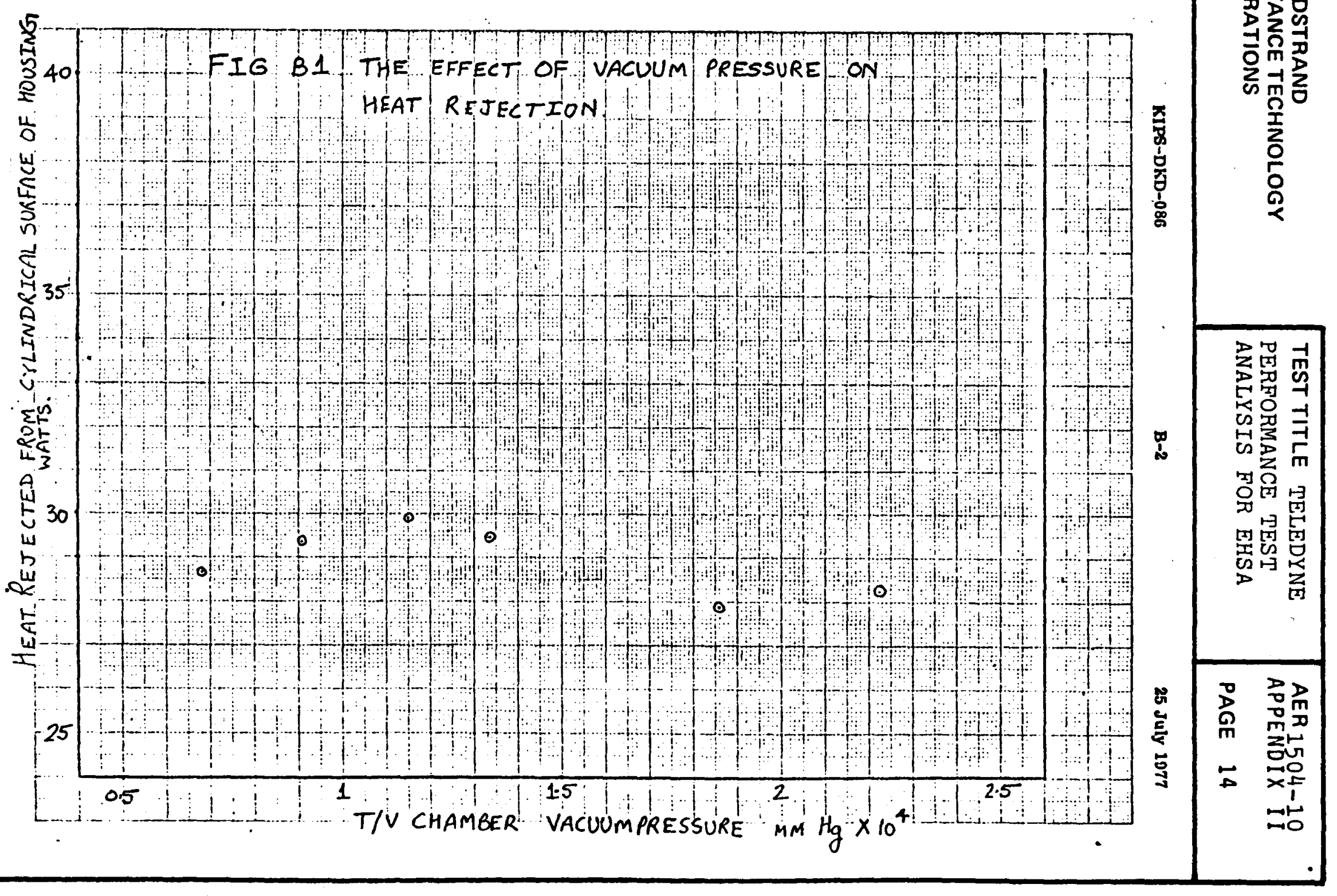




\section{PAGE 15}

KIPS-DKD-086

B-3

25 July 1977

\section{Heat Losses From the EHSA End Covers}

It is assumed that heat losses from the top and bottom surface of the EHSA would be of the same order of magnitude. However, in reality the heat rejected from the bottom surface would be slightly less because of its relatively lower temperature.

Area of Min-K in contact with heat block $=.35 \mathrm{ft}^{2}$

Thickness of Min-K $=1.75$ in.

Thermal conductivity of Min-K $=0.0085 \mathrm{Btu} / \mathrm{hr}-\mathrm{ft}-\bullet \mathrm{F}$

$$
Q=-k A \frac{\Delta T}{\Delta X}
$$

where, the symbols have their usual meaning.

\begin{tabular}{c}
$\begin{array}{c}\text { Average } \\
\text { Corrected } \\
\text { Heater Block } \\
\text { Temperature }\end{array}$ \\
\hline F \\
\hline 1404 \\
1396 \\
1394 \\
1407 \\
1406 \\
1404
\end{tabular}

\begin{tabular}{c}
$\begin{array}{c}\text { Average } \\
\text { Housing } \\
\text { Temperature } \\
-F\end{array}$ \\
\hline 273 \\
269 \\
270 \\
276 \\
277 \\
276
\end{tabular}

\begin{tabular}{l}
$\Delta T$ \\
\hline$F$ \\
\hline 1131 \\
1127 \\
1124 \\
1131 \\
1129 \\
1128
\end{tabular}

Q Watts

6.74

6.72

6. 70

6.74

6.73

6.72

Thus, the heat losses from the end ocvers are expected to be of the order of 7 watts. 
Heat Loss Through the Outlet Tube

Cross sectional area of the tube $=0.000388 \mathrm{ft}^{2}$

Thermal conductivity of stainless steel $=10 \mathrm{Btu} / \mathrm{hr}-\mathrm{ft}-{ }^{\circ} \mathrm{F}$

Heat conduction length $=8$ inches

$$
Q=-k A \frac{\Delta T}{\Delta X}
$$

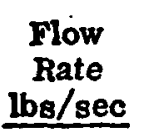

.0234

.0285

.0338

.0234

.0285

.0338

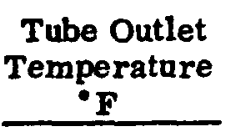

585

543

501

597

569

538

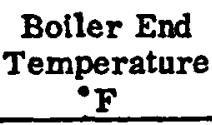

666

651

633

672

666

657

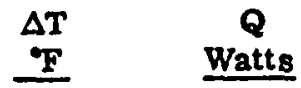

$81 \quad 0.14$

$108 \quad 0.18$

$132 \quad 0.23$

$75 \quad 0.13$

$97 \quad 0.17$

$119 \quad 0.20$

Heat conducted through the tube on the entrance side is not expected to be higher than that on the exit side because of lower temperature drop across the tube on the inlet olde. 
A SUMMARY OF HEAT LOSSES FROM EHSA

BASED ON VARIOUS COMPONENT TEMPERATURES

From the cylindrical portion of housing (Appendix B)

30 watts

From top of housing (inlet side) (Appendix B)

8 watts

From bottom of housing (outlet side) (A ppendix B)

8 watts

From electrical cable (Appendix D)

$<3$ watts

From Instrument cable (Ref. 3)

$<4$ watts

From Dowtherm tube (Appendix B)

$<1$ watt

Total

54 watt8 


\section{APPENDIX C}

\section{HEAT TRANSFER THROUGH MULTIFOIL INSULATION}

Based on the test data supplied by TECO, it is noticed that the gross heat flux through the 60 -layer multifoil insulation for the hot side temperature of $644^{\circ} \mathrm{F}$ is approximatcly 12 watts. In Figure $\mathrm{C}-1$ is plotted the heat flux through the insulation vs. effective thermal conductivity of the insulation for three different values of the effective emissivity of the insulation. The following equation was used for this purpose.

$$
Q=\frac{\sigma \text { Average }\left(T_{H}^{4}-T_{C}^{4}\right)}{(N+1)(2 / 6-1}+\frac{k_{e} A_{a v g}\left(T_{H}-T_{C}\right)}{\Delta X}
$$

For this graph the averaze hot and cold side temperatures as observed during the test were used. It is seen that for the effective emissivity value for the multifoll insulation reported in Ref. 1, the effective the rmal conductivity of the insulation is much higher than that reported in Ref. 1. Because of the difficulty experienced during the assembly operation in inserting the multifoil insulation in the housing it seems likely that portions of the insulation might have crumpled,creating thermal shorts.

Due to lack of correct property values of the multifoll insulation, it is difficult to estimate the heat lorses through the insulation. However, a rough estimation suggests this heat loss to be between 15 and 30 watts. 


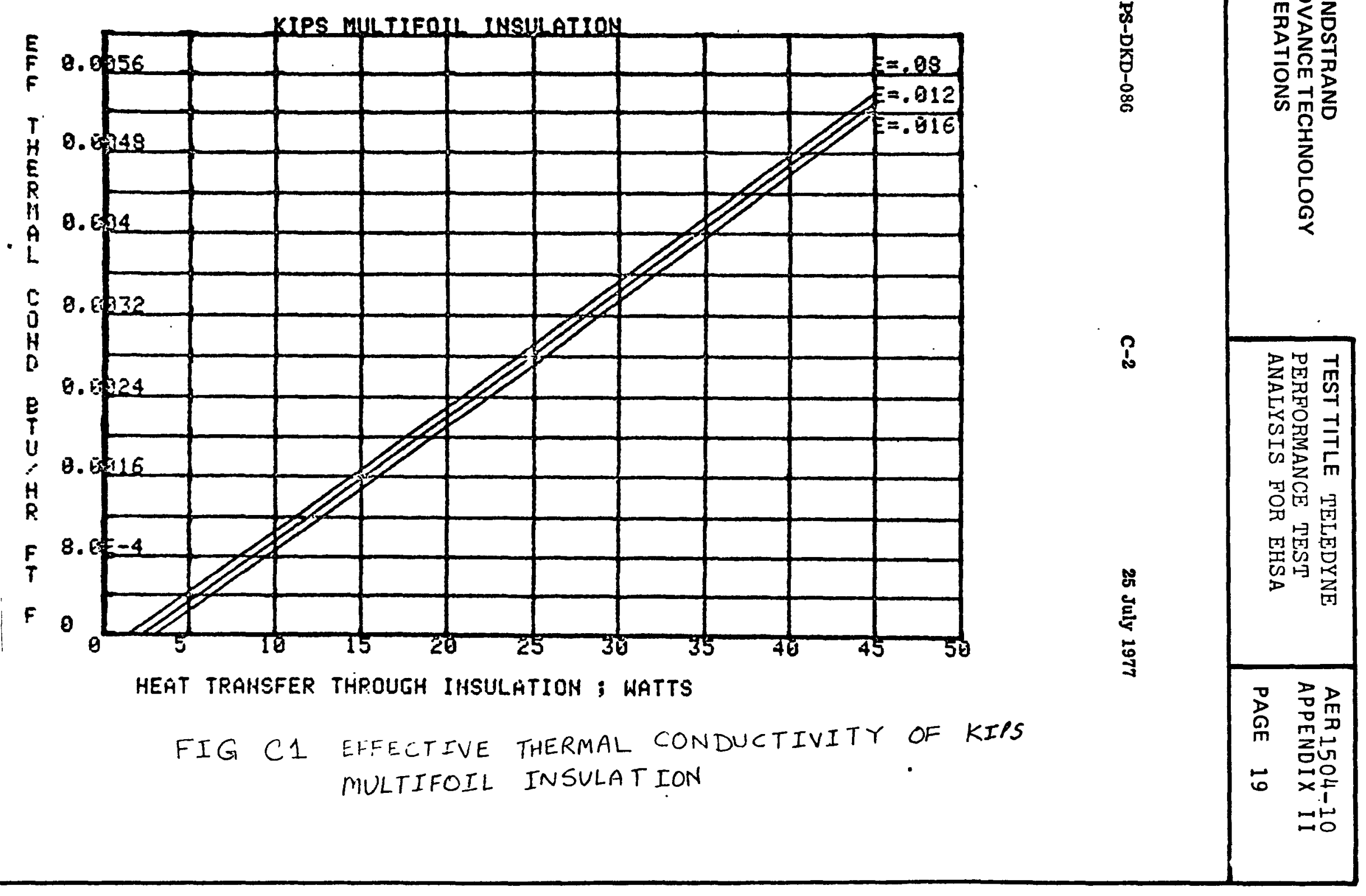




\section{APPENDIX D}

\section{KIPS HEATER LEAD AND POWER CABLE ANALYSIS}

In view of the recent acquisition of the test data the earlier KIPS heater lead and power cable analysis (Ref. 2) is updated and corrected for the inadvertent discrepencies which had entered therein. The principle of analysis remains basically the same as before; however, corrections were made for various configuration changes, thermal properties and environmental temperatures which are more commensurate with what has been observed during the test. A correction in the internal heat generation in the lead wires reveal that a considerably higher amount of electrical energy is being dissipated inside the EHSA by the heater lead wires.

The analysis is divided into two parts; one for the heater leads inside the EHSA and the other for the power cable external to the EHSA. The analysis utilizes a forward difference technique, wherein the solution progresses from the end of the heater cartridges out to the connector. For various initial assumptions of heat conduction into the lead wires the temperature of the housing connector and heat conducted away from it were calculated and which are plotted in Figure $\mathrm{D}-1$. The analysis was repeated for the cable outside the EHSA and the two solutions were matched such that the far end of the cable is in thermal equilibrium with its surroundings, namely the vacuum chamber wall. The program code and the typical solution outputs are attached herewith.

It is found that under the present setup the housing connector runs at a rather high temperature of $560^{\circ} \mathrm{F}$. One of the difficulty in determining this temperature exactly Is due to the unavailability of the thermal properties of the Deutsch connector. Nevertheless, this temperature is relatively less sensitive to these properties. The heat conducted out through the cable (heat loss from the EHSA) is about 1.3 watts. The Internal heat generation in the lead wires inside the EHSA is approximately 70 watts. It is seen from Figure D-2, that this heat generation is relatively insensitive to the
housing connector temperature. 
SUNDSTRAND

ADVANCE TECHNOLOGY

OPERATIONS
TEST TITLE TELEDYNE

PERFORMANCE TEST

ANALYSIS FOR EHSA
AER $1594-19$
APPENDIX I

PAGE 21

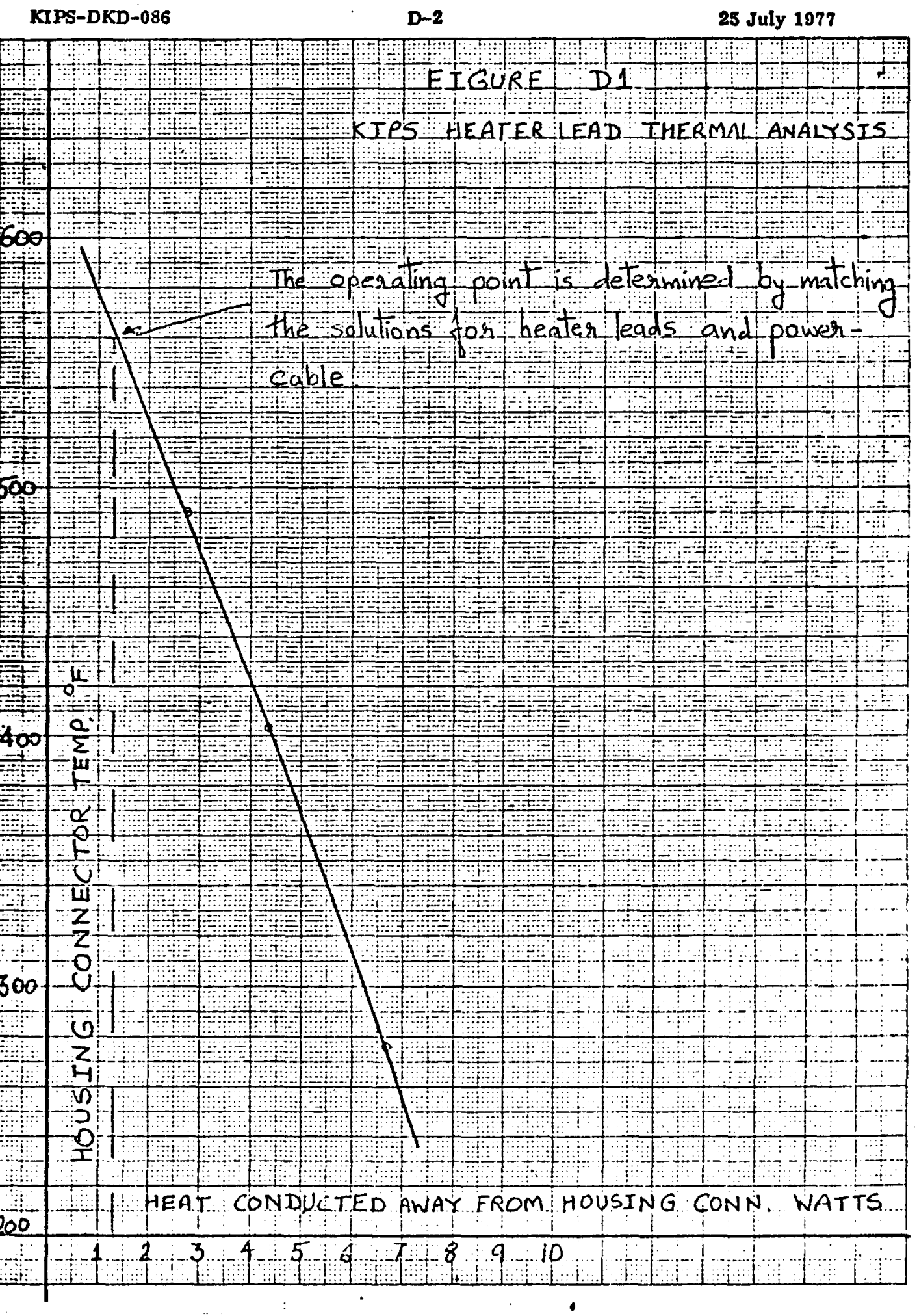


PERFORMANCE TEST

ANALYSIS FOR EHSA
APPENDIX II

PAGE 22

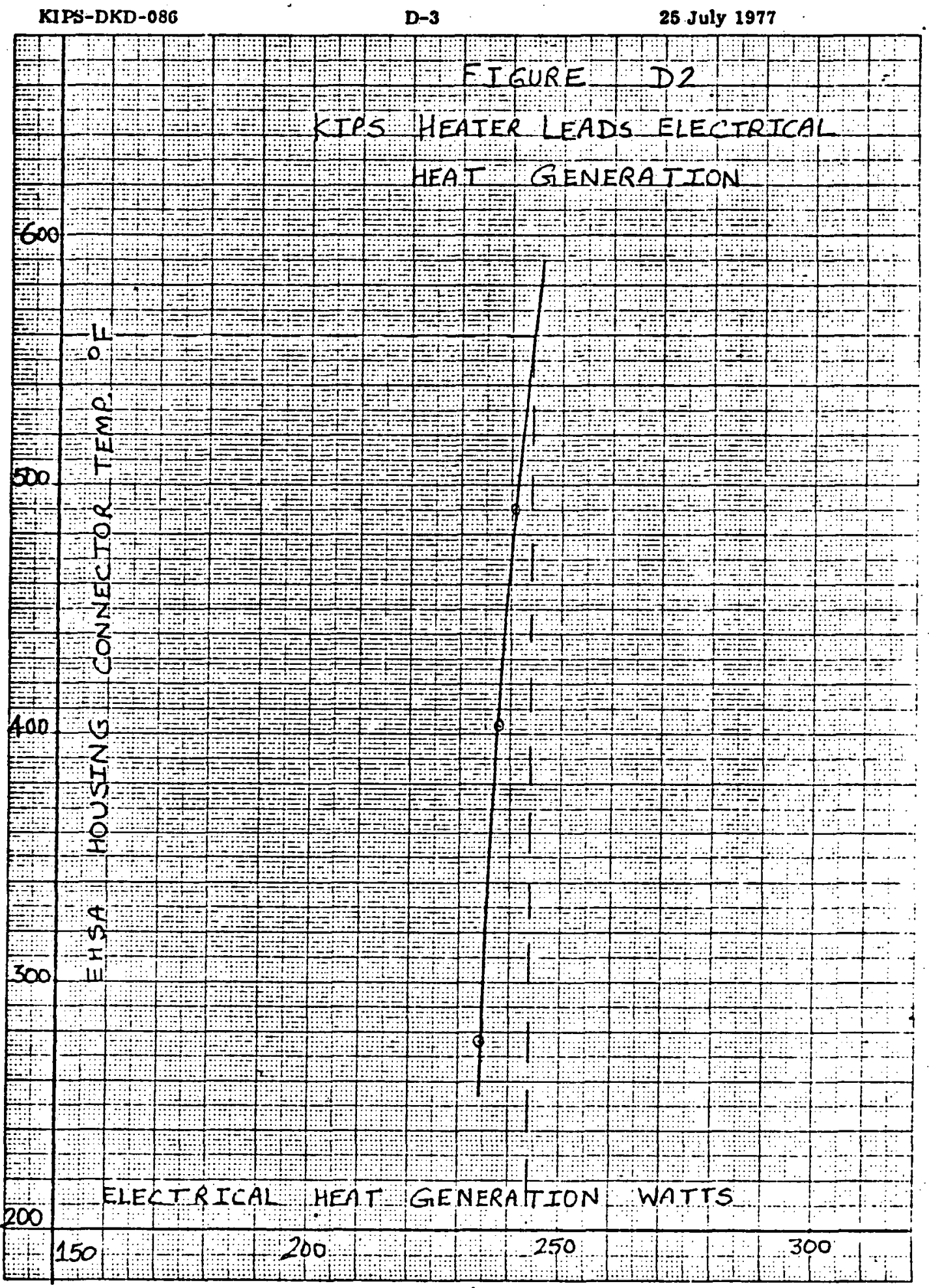




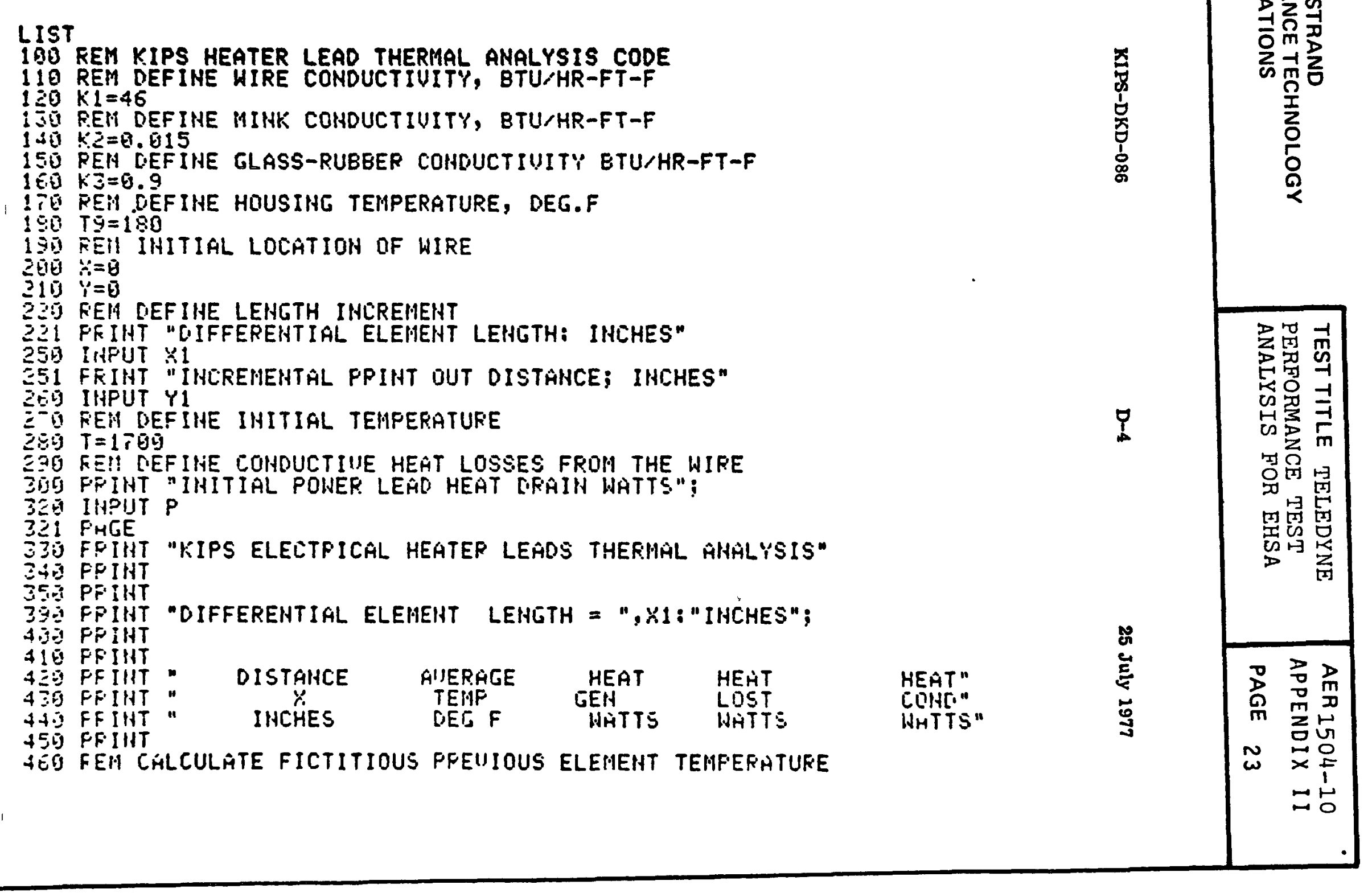


$47 \theta T \theta=T+3.412 * P * X 1 / 12 /(K 1 *(\theta .032 \uparrow 2 * 26 * P 1 / 4) / 144)$

48. REM FIND HERT GAIHEO FROM PREUIOUS ELEMEHT

400 Q1 $=K 1 *(P I * 0.032+2 * 26,4) *(T B-T)<(\% 1 * 12)$

5ZOU REN CALCULATE HEAT GENERATED IN DIFFERENTIAL ELEMENT

$53206=8$

510 IF T>1000 THEH 540

$520 \quad 0=38+0.195 * T$

530 CO TO 550

$540=225+0.09 *(T-1000)$

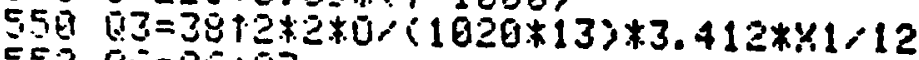

$55200=06+03$

SÜB REN FIHO HEAT LOST TO SURROUUHOIHGS

Elī IF $X Y 2$ THEH 040

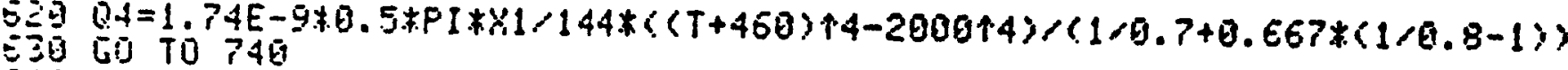

6.49 IF $\% 5.5$ THEN 670

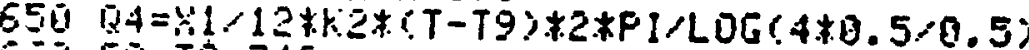

E.0 GT TO 748

ETE IF $P>14$ THEH 70 G

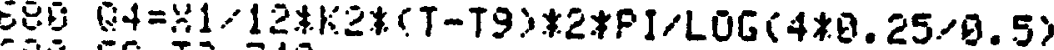

8350 TO TO 740

700 If $O 14.25$ THEH $8 E$ E

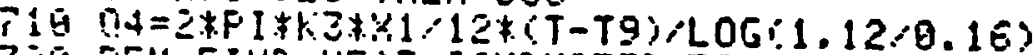

?EE EEH FIHO HEHT COHOUCTEO TU HEXT ELEMEMT

$P+\theta 02=01+03-04$

FEIS REN SET CUREENT TO PREUIOUS ELEMENT TEMPERGTURE

$T E T$ TIS=T

$T T=T E-1024.1-120(K 1 *(0.03212 * 2 E * F 1,4)(144)$

750 IF $\therefore$ THEH 19

7E: I!HEE $50.20,140.20,50,40,50,40,50,40$

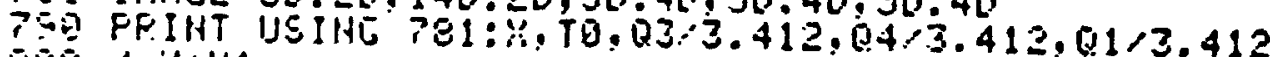

Ting $i=r+1$

S!g REN RESET $\because$

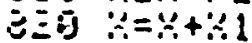

ZJÜ FEN SET HEAT OUT OF THIS ELEHENT EQUUAL TO HEAT INTO HEXT ELEMENT 
$840 Q 1=Q 2$

SEO GO TO 510

S?O PRIHT "CUMULATIUE HEAT GENERATIOH =",06: : ,

880 ENO

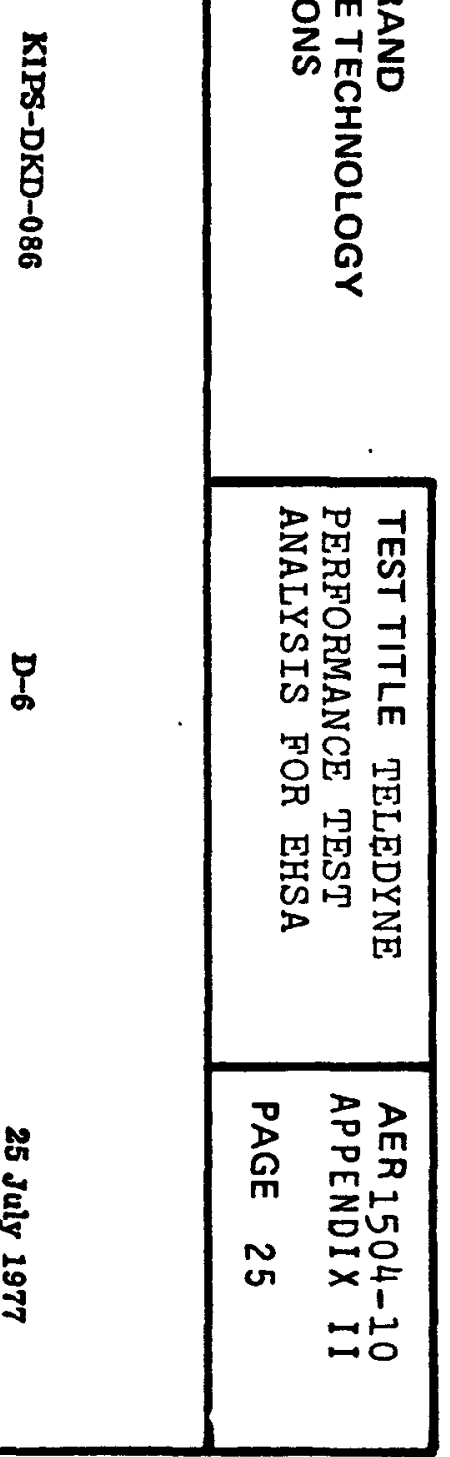


KIFS ELECTRICAL HEATER LEADS THERMAL ANALYSIS

DIFFERENTIAL ELEMENT LENGTH =

DISTANCE

0.00

0.25

0.55

1.60

1.25

1.59

$1 ., 15$

2.90

3.25

政

3.90

3.25

3.50

3.75

4.9519

4.25

4.58

4.75

5.95

5.25

$5 .=5$

8.00

E. 25
AUERAGE

DEG $F$

1700.00

1650.89

1024.54

1611.73

1 EOE. 11

1612.48

1E.EE. 28

1654.23

1วดิ 13

1772.95

1832.61

185.67

1935.36

1968.47

1999.41

2023.18

2139.78

2049.21

2051.47

2146.57

2034.50

2015.26

1958.86

1959.06

$19=9.94$

19131.38

\subsection{INCHES}

HEAT

WATTS

0. 0653

Q. 1643

日. 9538

0. 0635

0. BE35

Q. 86.36

0. 11638

0. 0.44

ด. 0655

6. 60.8

ด. 0605

0. $\operatorname{ces} 1$

ด. 9700

6. 9708

B. 715

0.61

6. 19723

ต. -25

G. 1824

Q. $07=2$

Q.071

5. 6712

5. 5700

ด. ติำ

ธ. 0655

\section{HEAT}

WATTS

ด. 2514

0.1685

0.1255

0. 1056

E. 1000

0.1067

6. 1284

0.1735

19. 202

6. 6.34

0.0354

‥ 日35.3

日. 0371

9.6375

6. 0383

0. 1356

6. 0358

8. 1388

6. 9787

6. 1355

日. 153

6. 6375

6. 1.79

8. $67=0$

ต. 19715
HEAT

COHD

WATTS

\subsection{5}

3.4515

1. 7320

6.752

$-9.5150$

- $9.79 \div 5$

$-1.825$

$-3.4833$

$-5.4545$

$-5.9545$

$-5.384$

$-4.0650$

$-3.95$

-3.

- 2.58

$-1.915$

$-1.2359$

- 1.5654

6. 107

6. 7805

1.45 .41

2. 127 .

2. 5014

2.7830

2. 7o 4

2.5030 


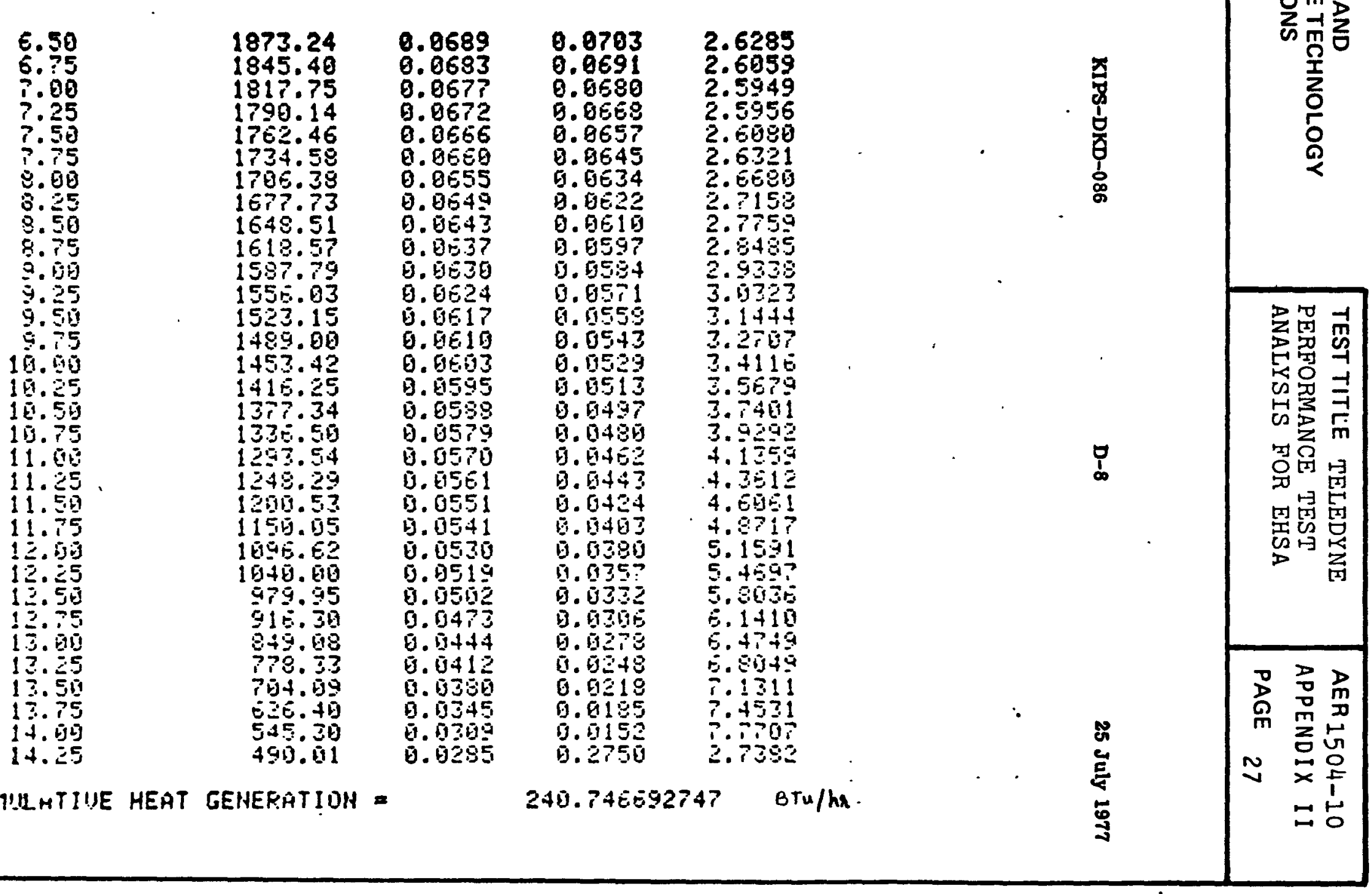


LIST

IEI REM KIPS HEATER CABLE THERMAL GHALYSIS

110 KEM CEFINE WIRE COHDUCTIUITY, BTU,HR-FT-F

$125 \times 1=230$

13G REM GEFIHE CABLE EFFECTIUE RADIAL COHDUCTIUITY, BTU-HRAFT-F

$140 ! C=0.16$

1SE FEM DUEF IHE GLASS RUEEER COHDUCTIUITY, BTU八HR-FT-F

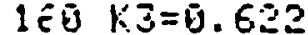

170 REM CDEFIHE SPACE TEMPERATURE,DEG. F

$1=59=130$

135 PEH CHAMEER HALL TEMPEPATUFE OEG $F$

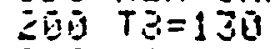

II FEN DEFIHE IHITIAL LOCATION OH WIRE

$2 \geq 0 \quad y=0$

23日 $Y=0$

ZT REM DEF IHE LEHGTH IHCREMEHT

25O FEIHT "DIFFEREHTIAL ELEMEHT LEHGTH, FEET";

QEE IHFUT $Z 1$

ZTG REM CEFIIIE PRIHT IIITERUAL

ZQE PFIHT "IHCFEMEHTAL PRIHT DUT OISTAHCE, FEET ";

¿̇̈ IISPLTT YI

30D FEH CEFIHE IHITIAL TEMFERATURE, DEG. $F$

31 FEIHT "HOUSIHE COHHEITOR PIH TEMFERATURE, DEG. F":

?EO IHFUT T

3IH FEI! [EFIHE IHITIRL COHOUCTIUE HEAT LOSS THROUCH WIRES

3:T FEIHT "TUTAL IHITIAL CABLE HEAT ORAIH, WATTS":

$35 \bar{S}$ IIIFUT $P$

$300 \quad[=-0.051$

JE! FMISE

3:G CFIHT "KIPS UACUUM CHGMEER CAELE THERMAL AHALYSIS"

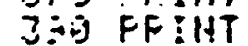

4. ER:IITT " OIFFEPEHTIAL ELEMEHT LEHGTH $=", X 1 ;$ "FEET"

419 FEIHT " WIFE OIAMETER $=", 0$; "IHCHES"

4 AU FRIHT " IHITIAL CÄBLE HEAT ORAIH = ",P: "WHTTS" 


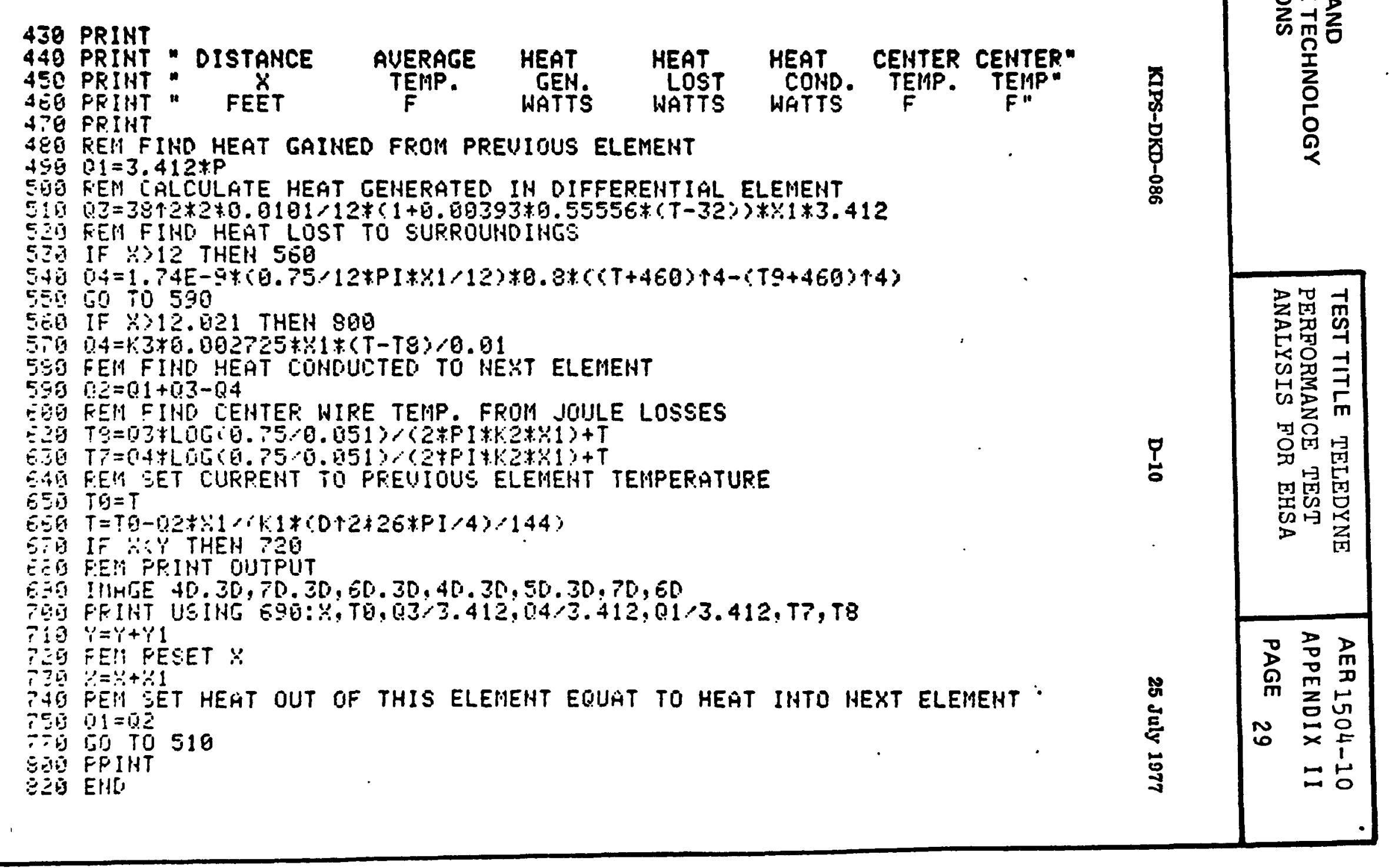


KIPS UACUUA CHAMBER CABLE THERMAL ANALYSIS

\begin{tabular}{|c|c|c|c|c|c|c|}
\hline \multicolumn{3}{|c|}{$\begin{array}{l}\text { OIFFEREHTIAL ELENENT LEHETH = } \\
\text { WIRE OIAMETER = O. OSIIHCHES } \\
\text { IHITIAL CAELE HEAT DRAIH = }\end{array}$} & \multicolumn{3}{|c|}{$\begin{array}{l}0.05 F E E T \\
1.2751 \text { TWATTS }\end{array}$} & \multirow[b]{2}{*}{$\underset{f \text { CENTER }}{\text { TEMP }}$} \\
\hline $\begin{array}{l}\text { OISTGIHCE } \\
\text { FEË }\end{array}$ & $\begin{array}{l}\text { AUERAGE } \\
\text { TEMP. } \\
\text { F }\end{array}$ & $\begin{array}{l}\text { HEAT } \\
\text { EEHH. } \\
\text { WATTS }\end{array}$ & $\begin{array}{l}\text { HEAT } \\
\text { LOST } \\
\text { WATTS }\end{array}$ & $\begin{array}{l}\text { HEAT } \\
\text { CÖHD. } \\
\text { HATTS }\end{array}$ & $\begin{array}{l}\text { CENTER } \\
\text { TEMP. } \\
\text { F }\end{array}$ & \\
\hline 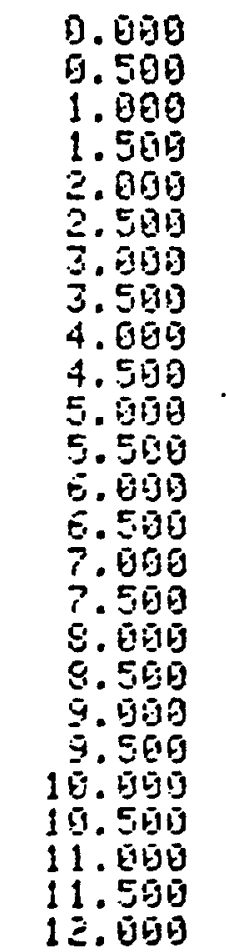 & 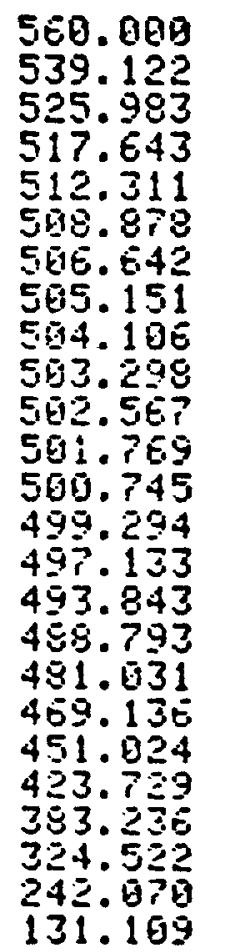 & $\begin{array}{l}0.262 \\
0.256 \\
0.253 \\
0.259 \\
0.249 \\
0.248 \\
0.247 \\
0.247 \\
0.247 \\
0.247 \\
0.246 \\
9.246 \\
0.246 \\
0.246 \\
0.245 \\
0.244 \\
0.243 \\
0.241 \\
9.238 \\
9.233 \\
9.225 \\
0.215 \\
0.199 \\
0.177 \\
0.149\end{array}$ & $\begin{array}{l}0.321 \\
0.292 \\
0.275 \\
0.254 \\
0.258 \\
0.254 \\
0.251 \\
0.249 \\
0.248 \\
0.247 \\
0.246 \\
0.245 \\
9.244 \\
0.242 \\
0.240 \\
0.235 \\
0.230 \\
9.221 \\
0.205 \\
9.159 \\
0.103 \\
0.128 \\
0.085\end{array}$ & 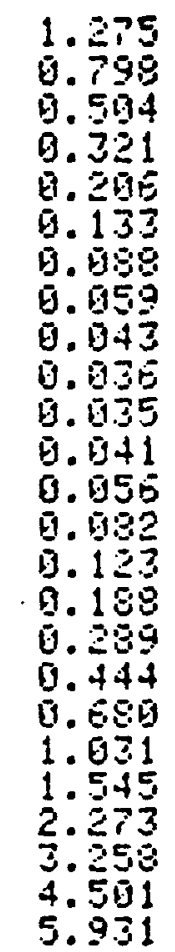 & 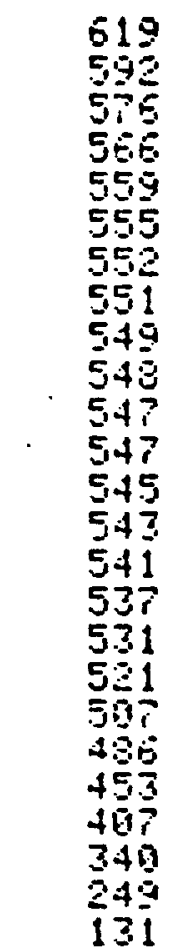 & $\begin{array}{l}688 \\
596 \\
572 \\
563 \\
558 \\
554 \\
552 \\
550 \\
549 \\
548 \\
545 \\
547 \\
546 \\
544 \\
542 \\
538 \\
533 \\
525 \\
512 \\
493 \\
465 \\
422 \\
361 \\
2.45 \\
153\end{array}$ \\
\hline
\end{tabular}

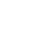

028

古第 


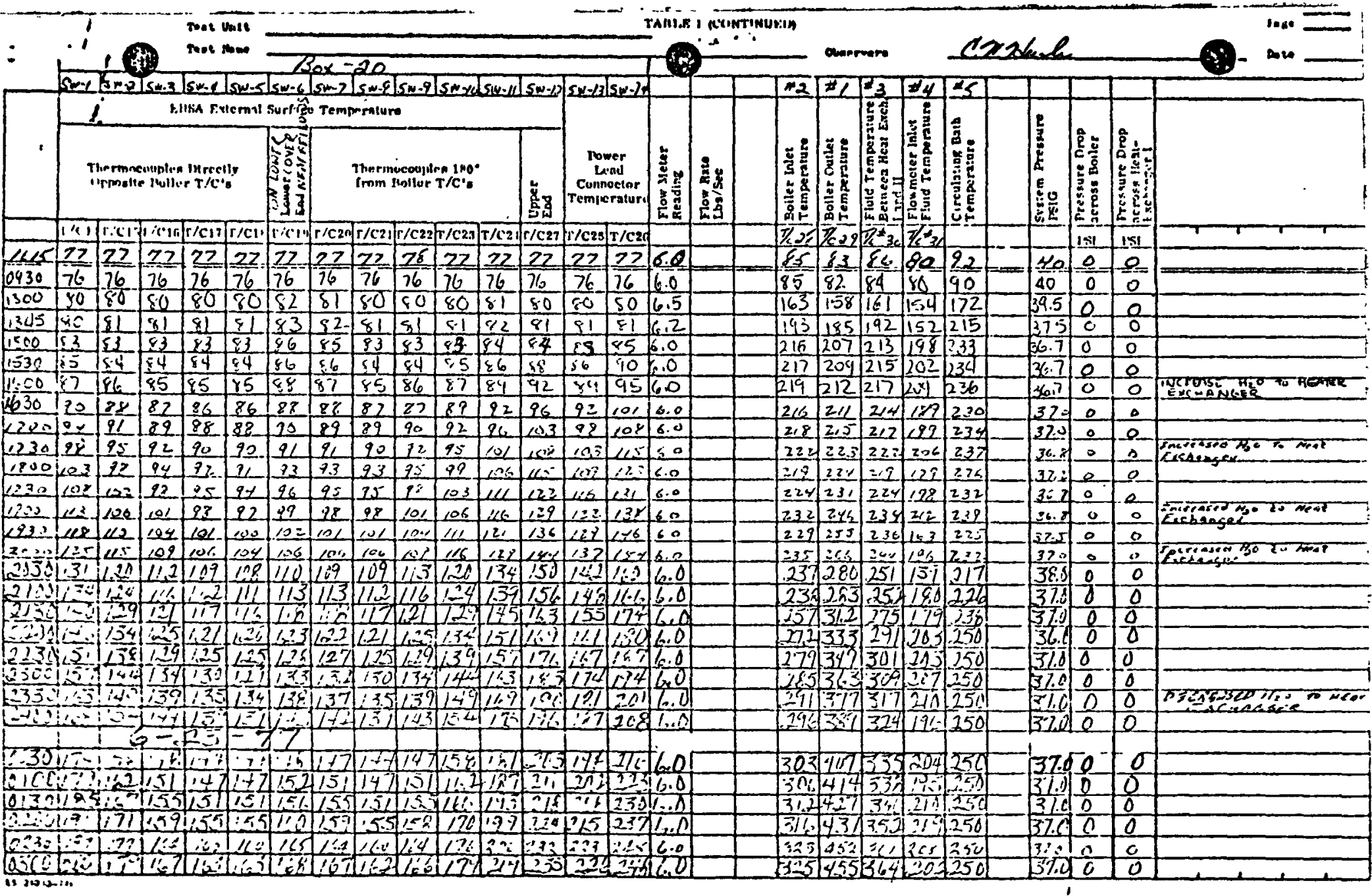




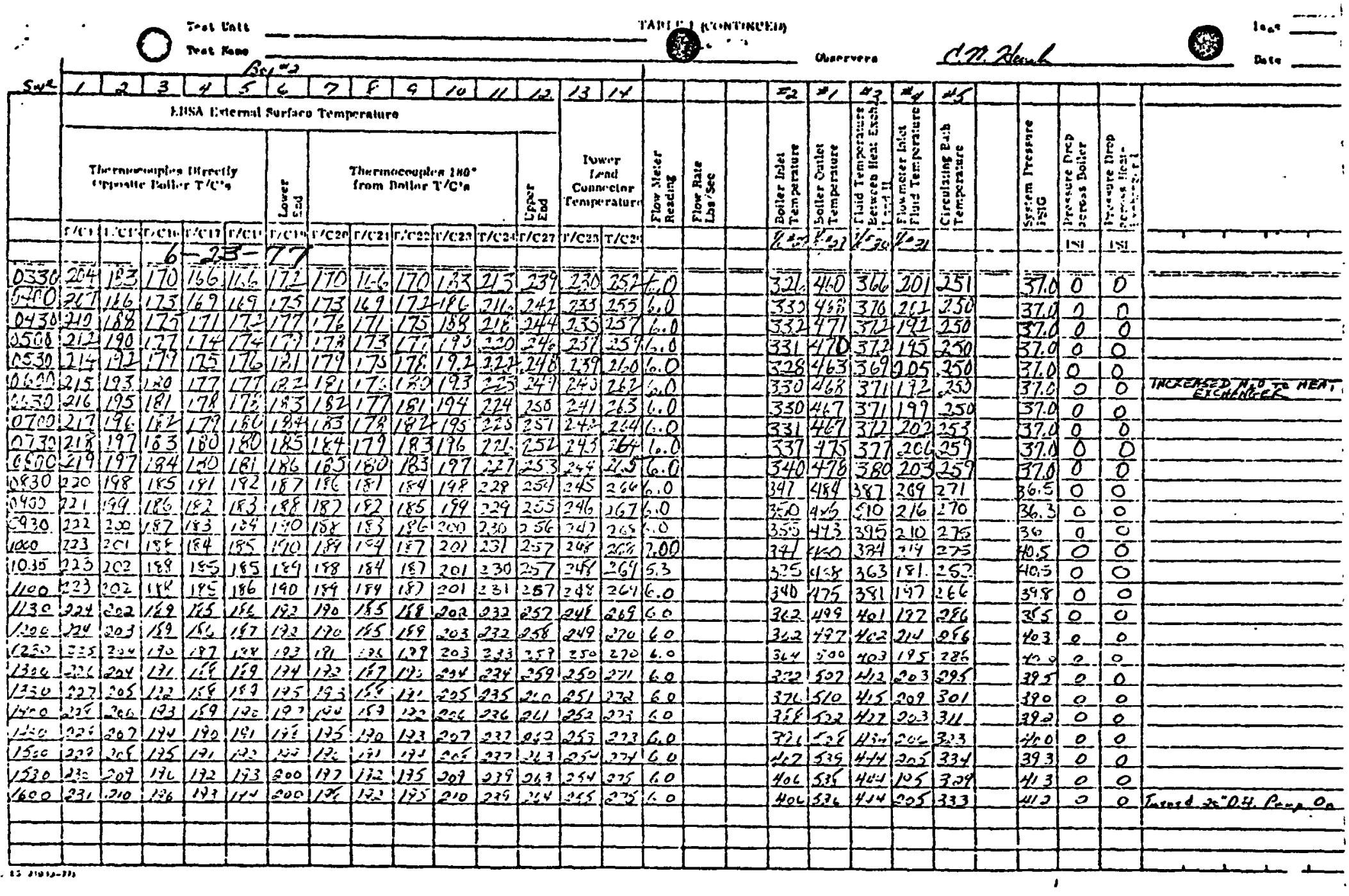

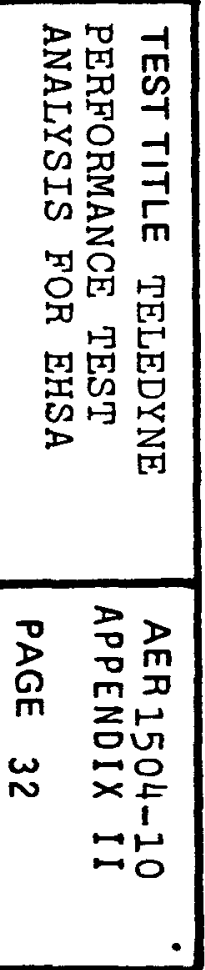




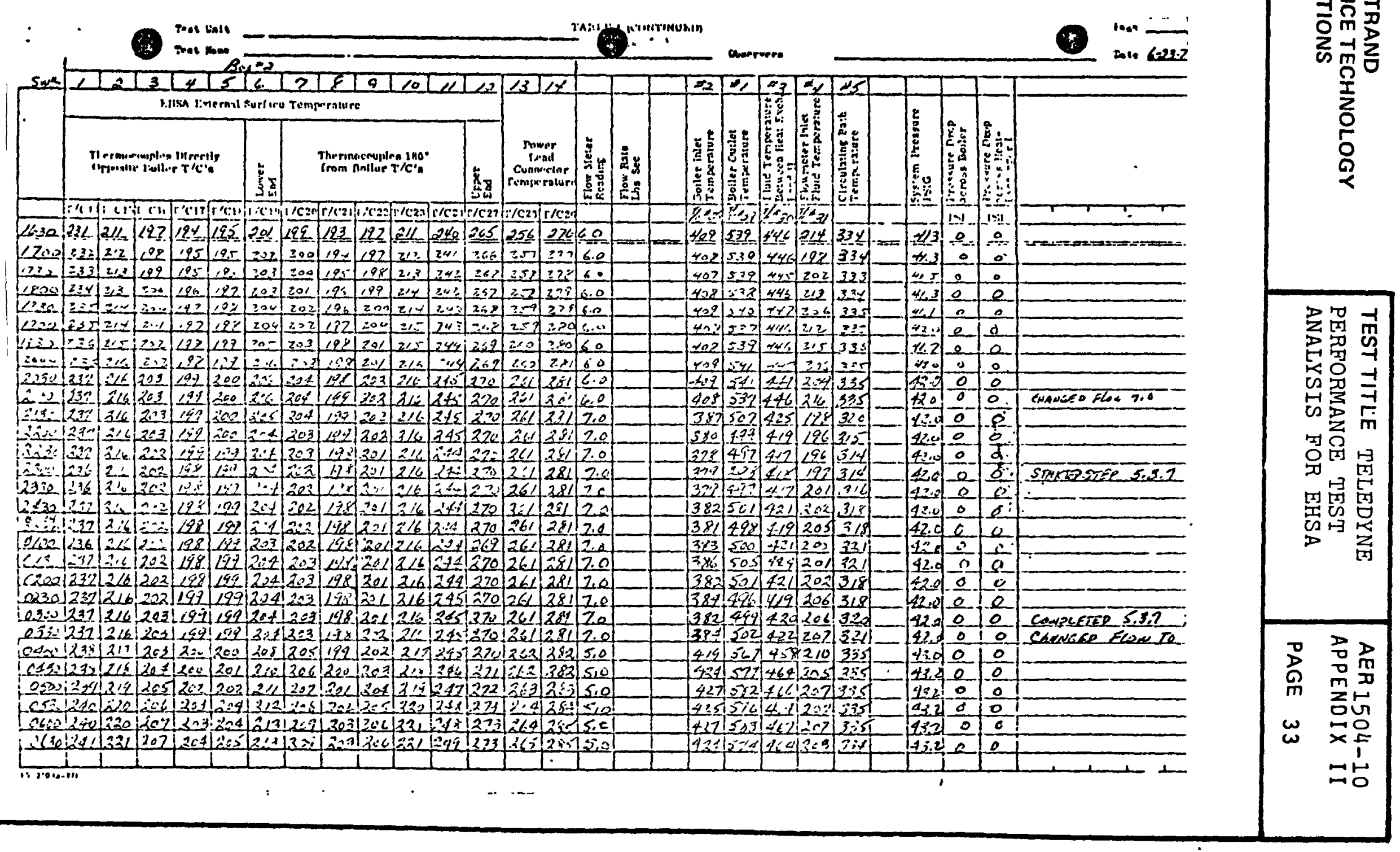




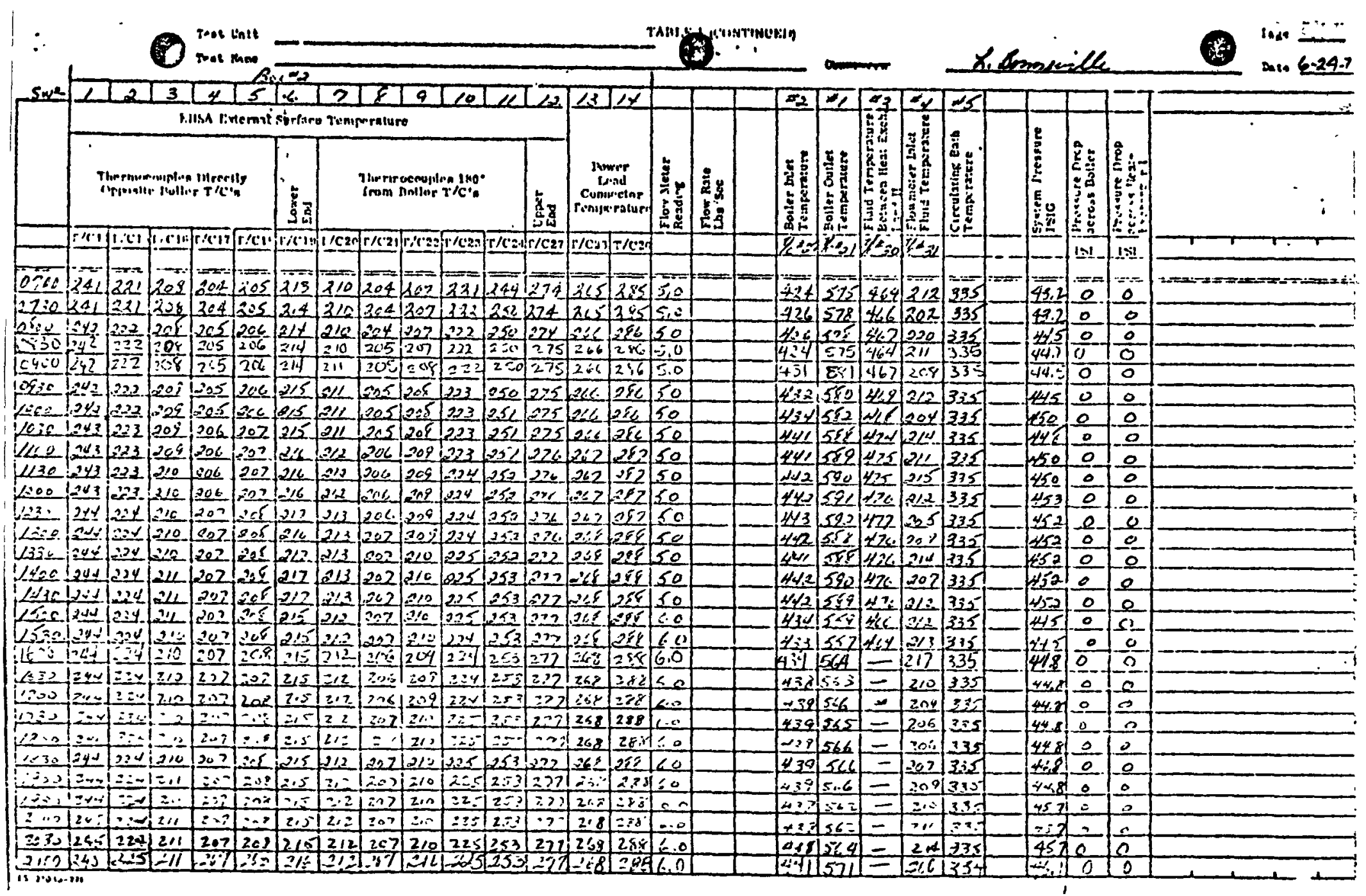

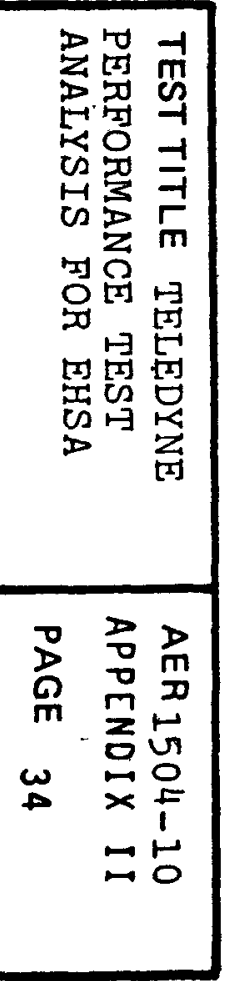




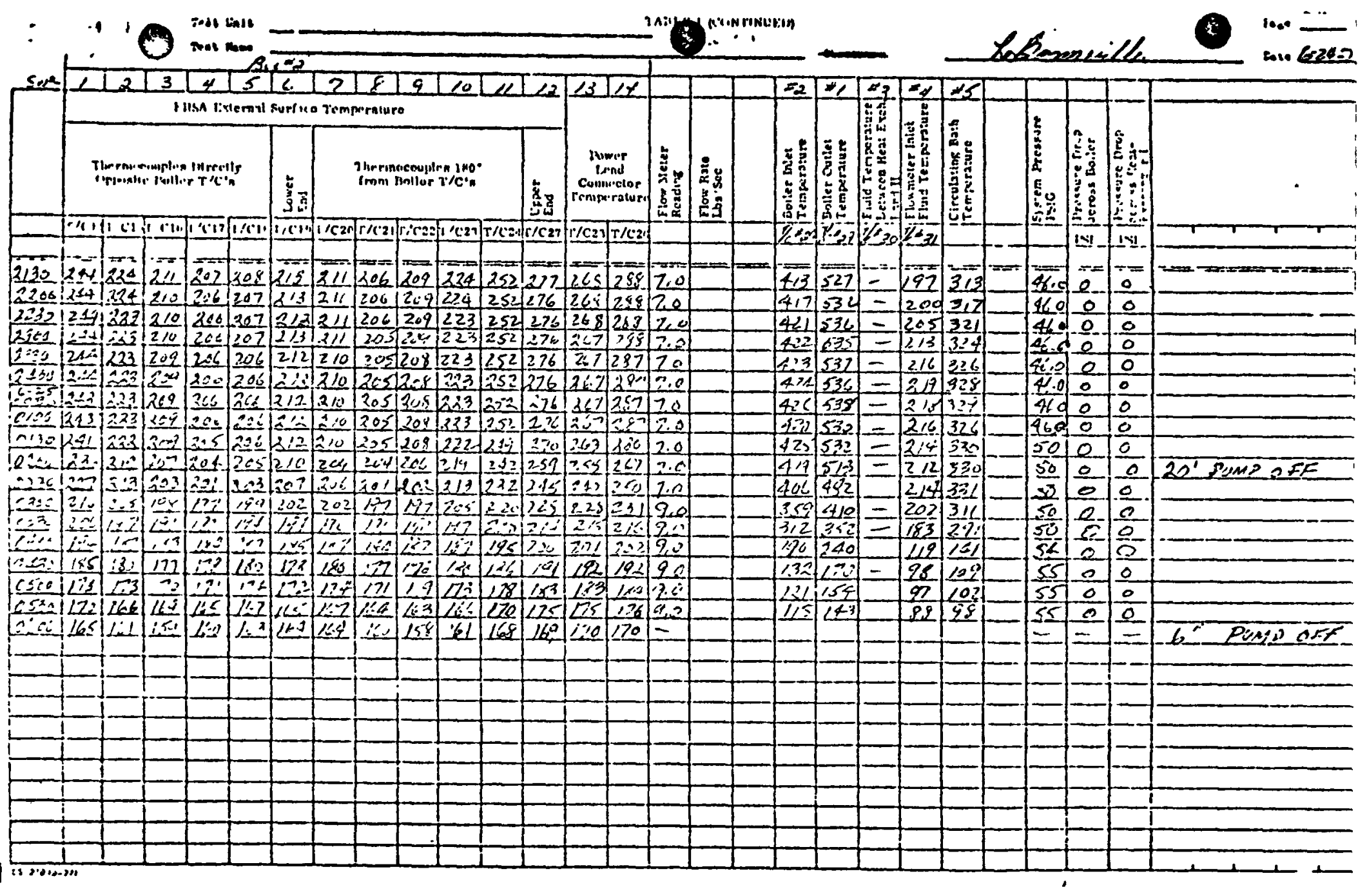

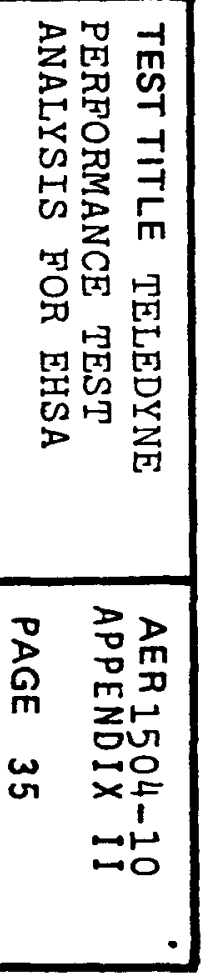




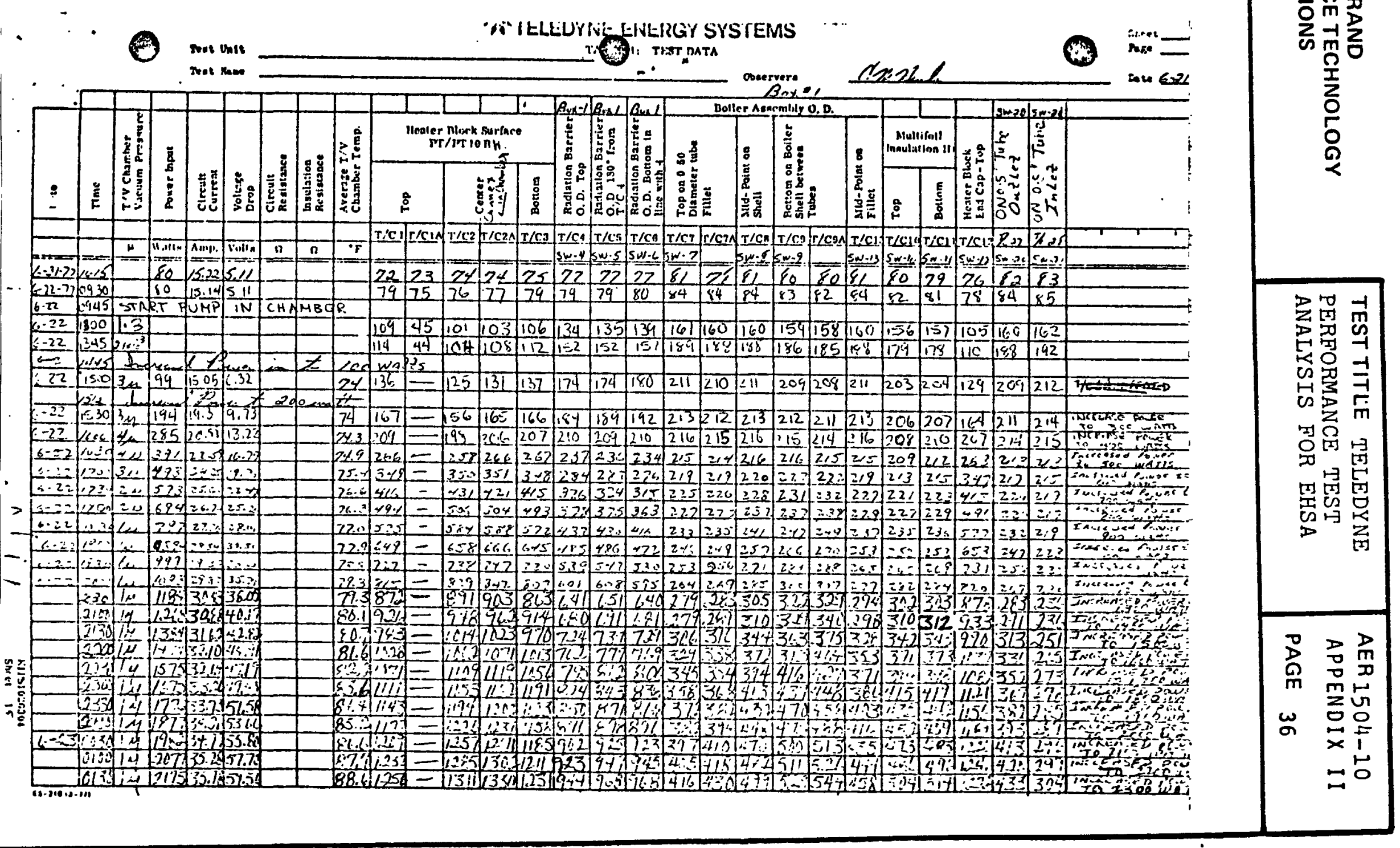




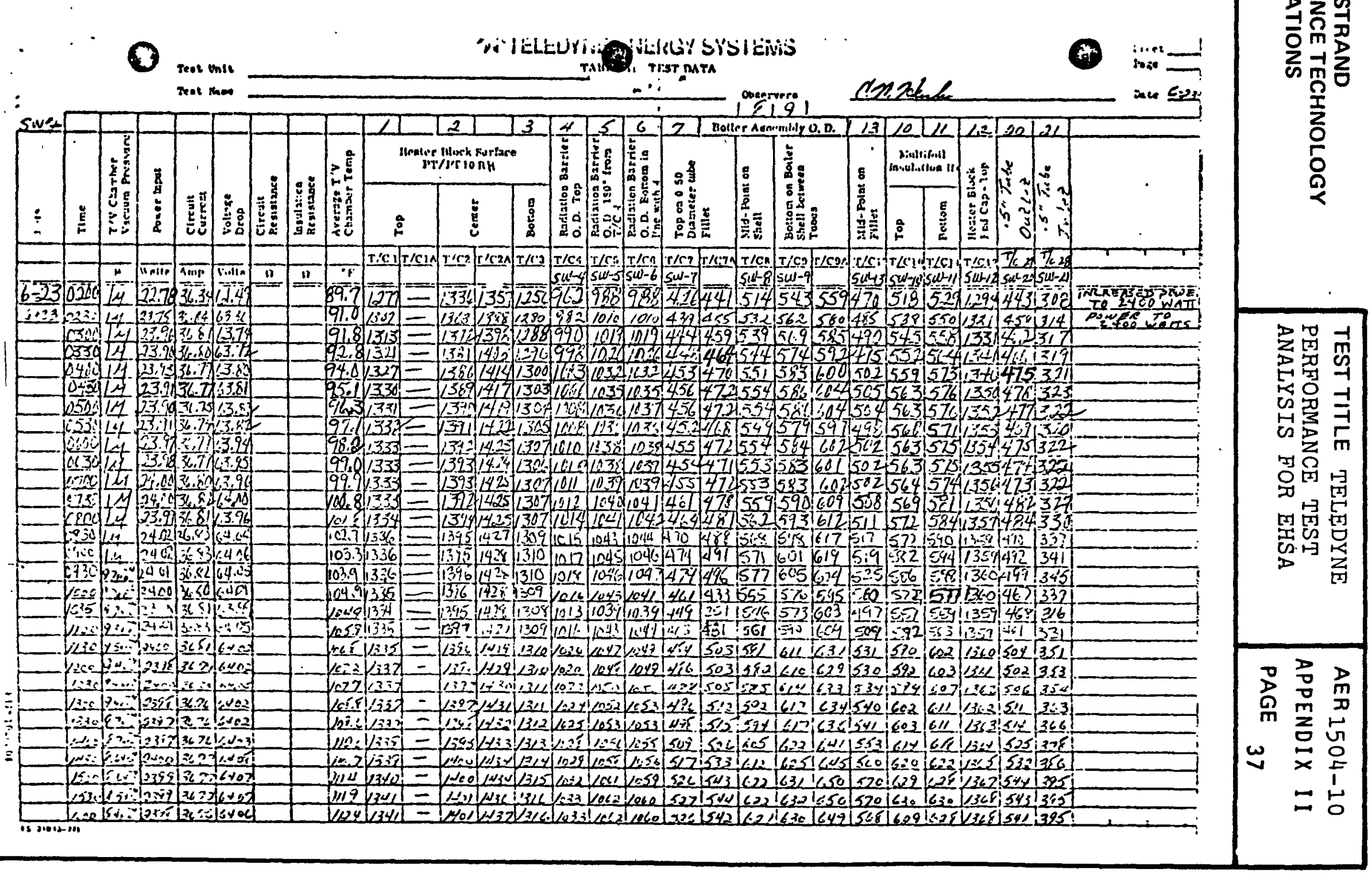




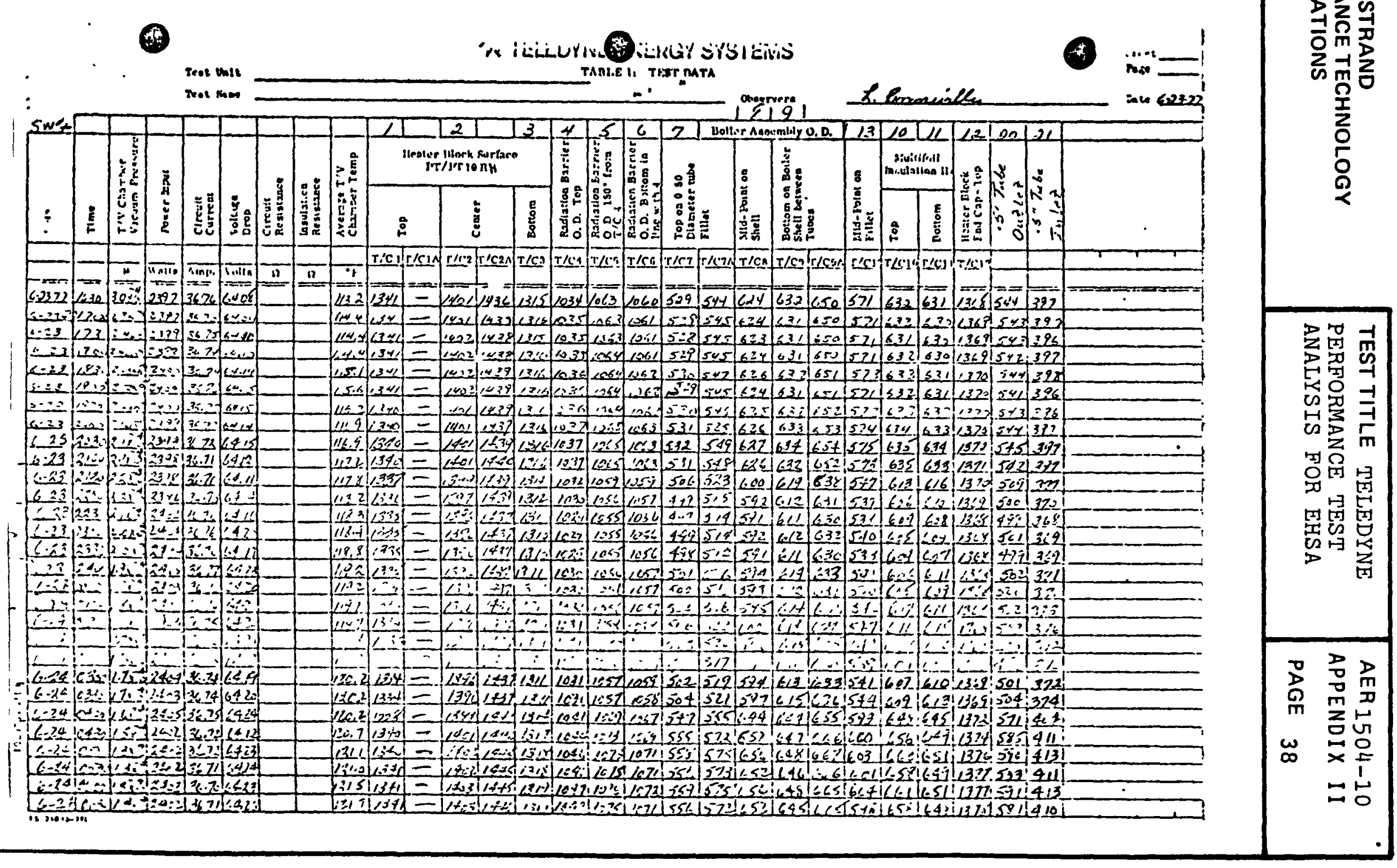




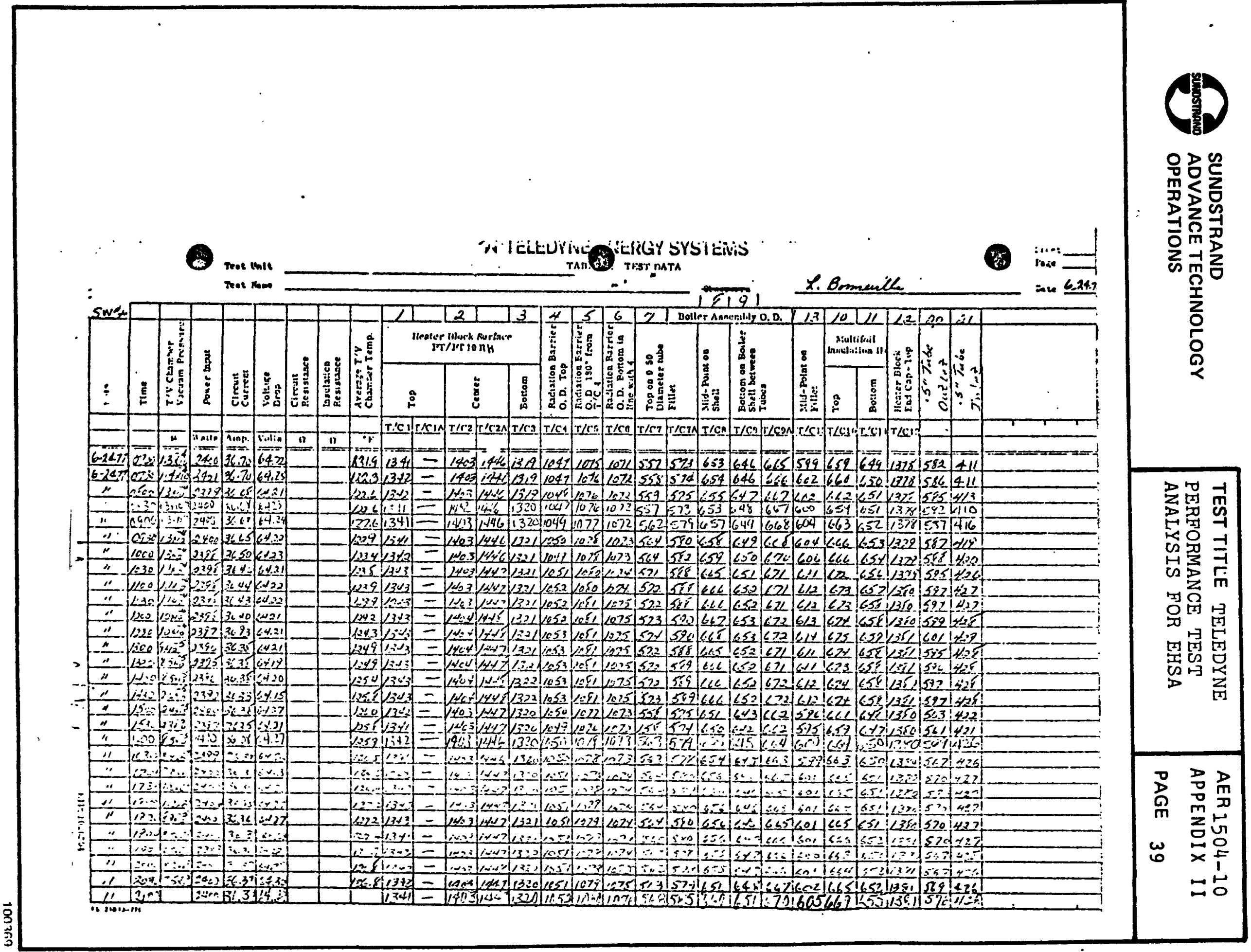




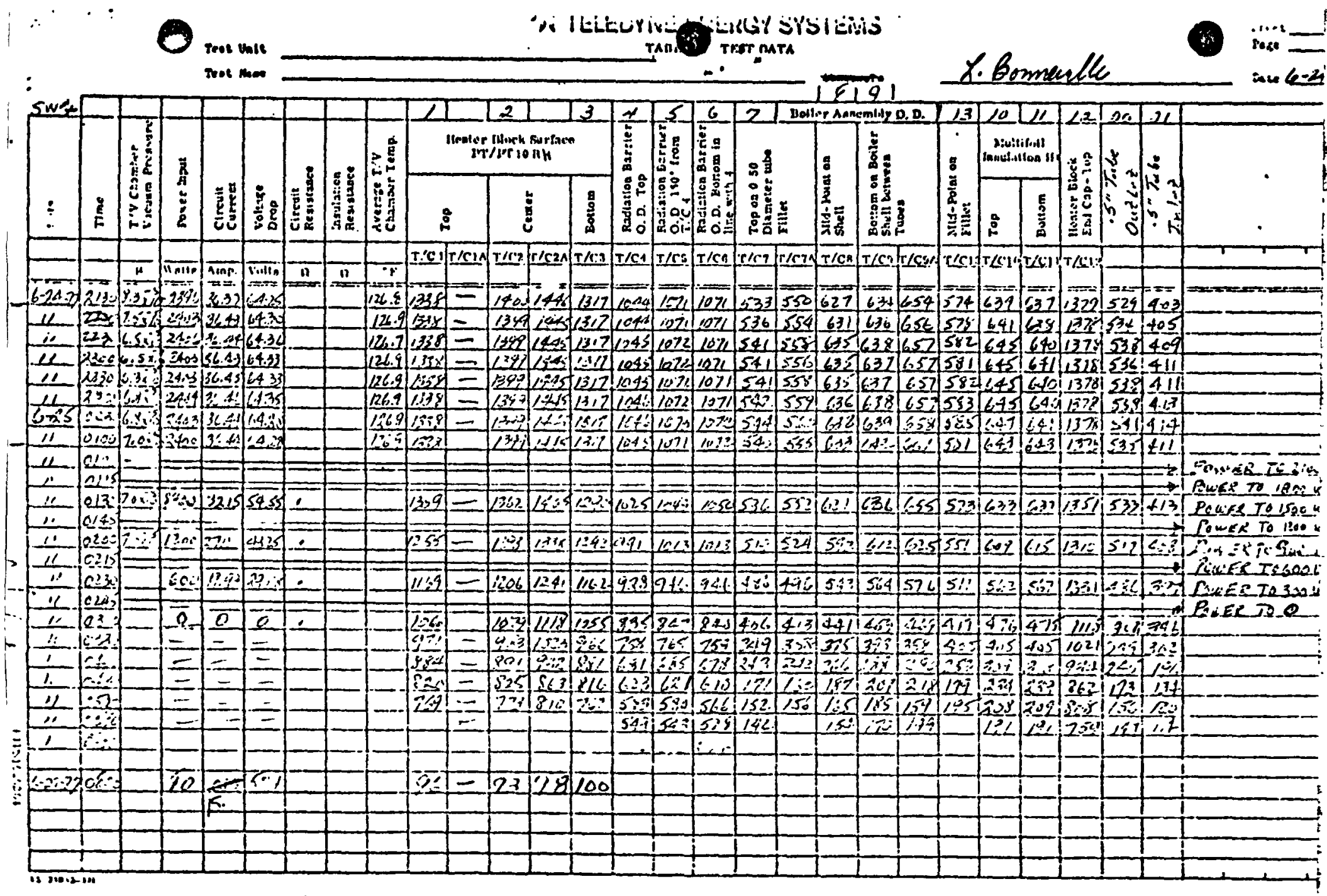

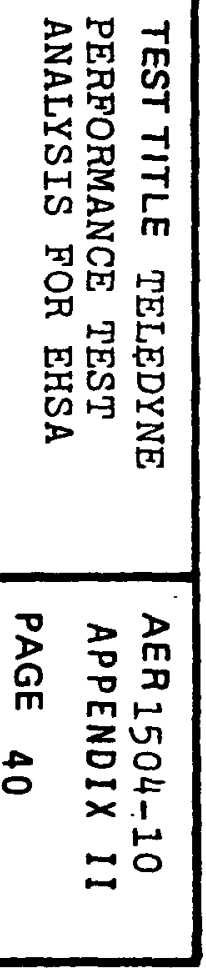




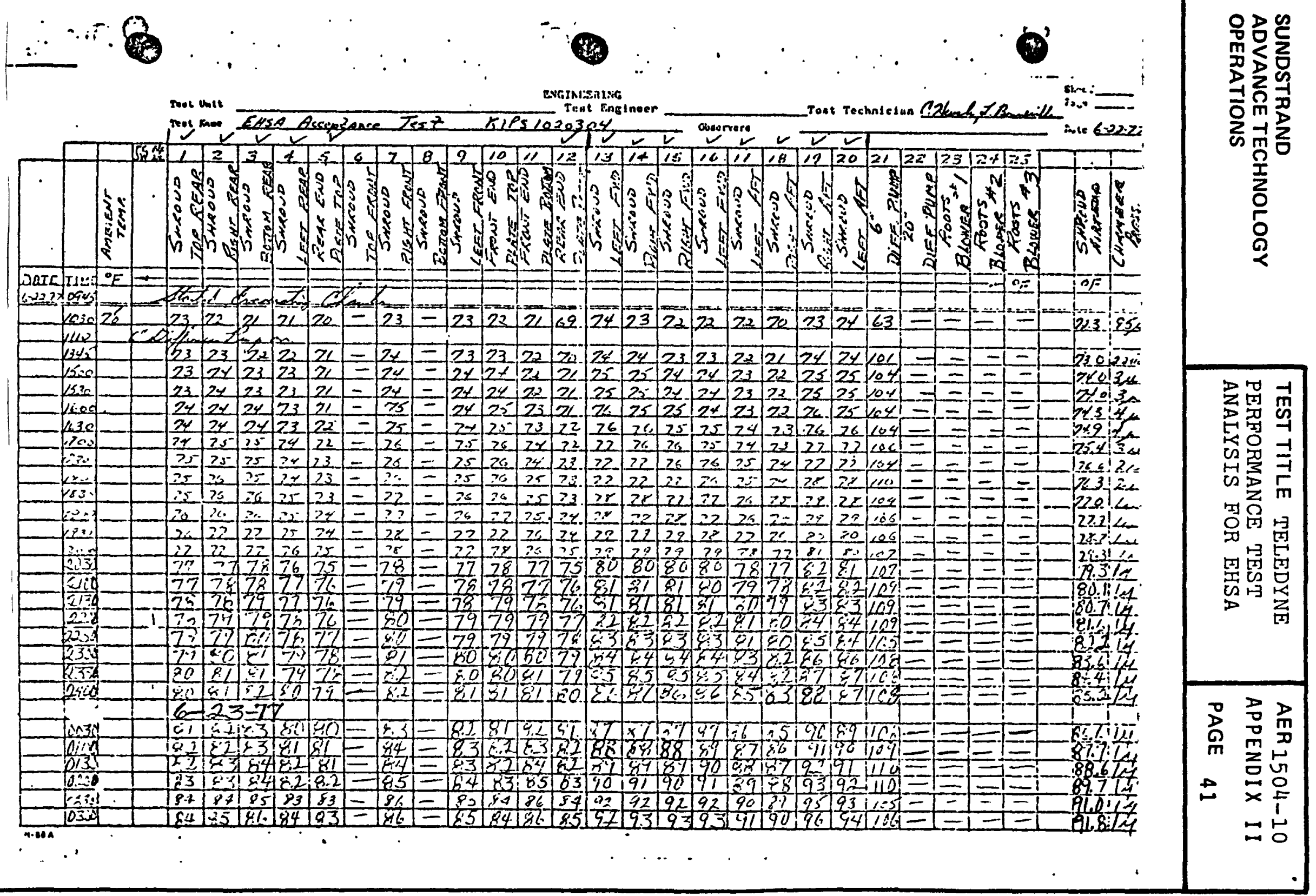




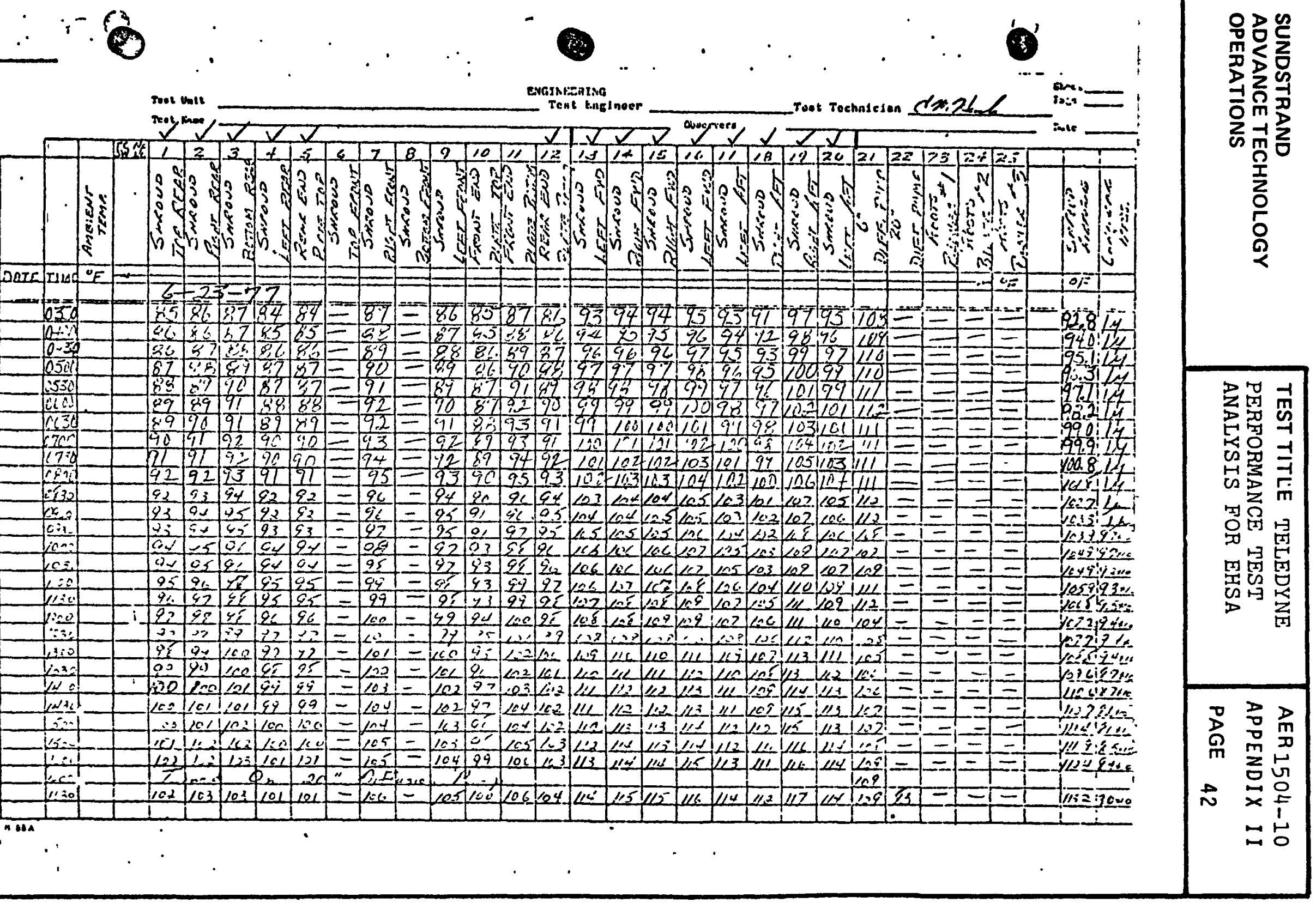




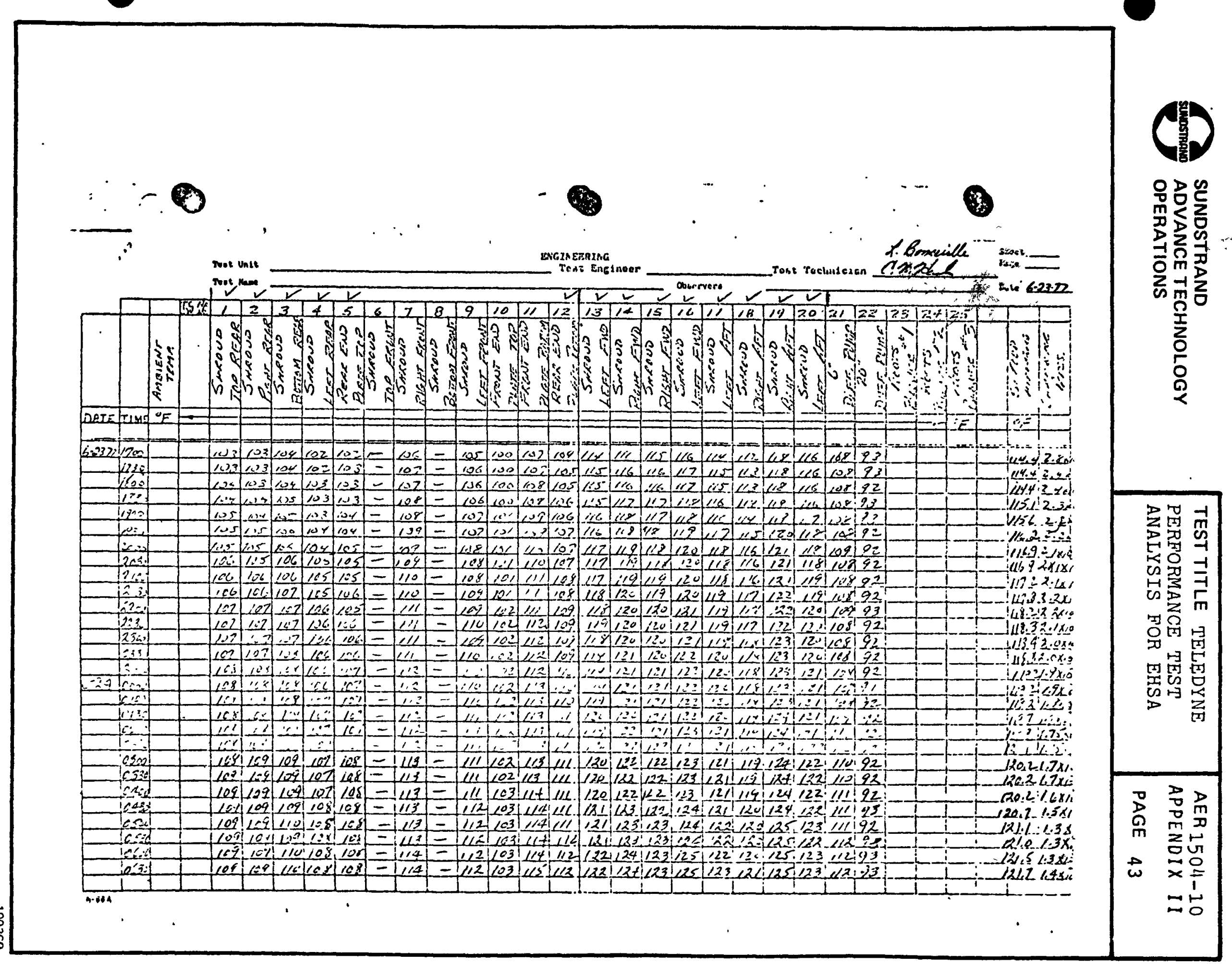




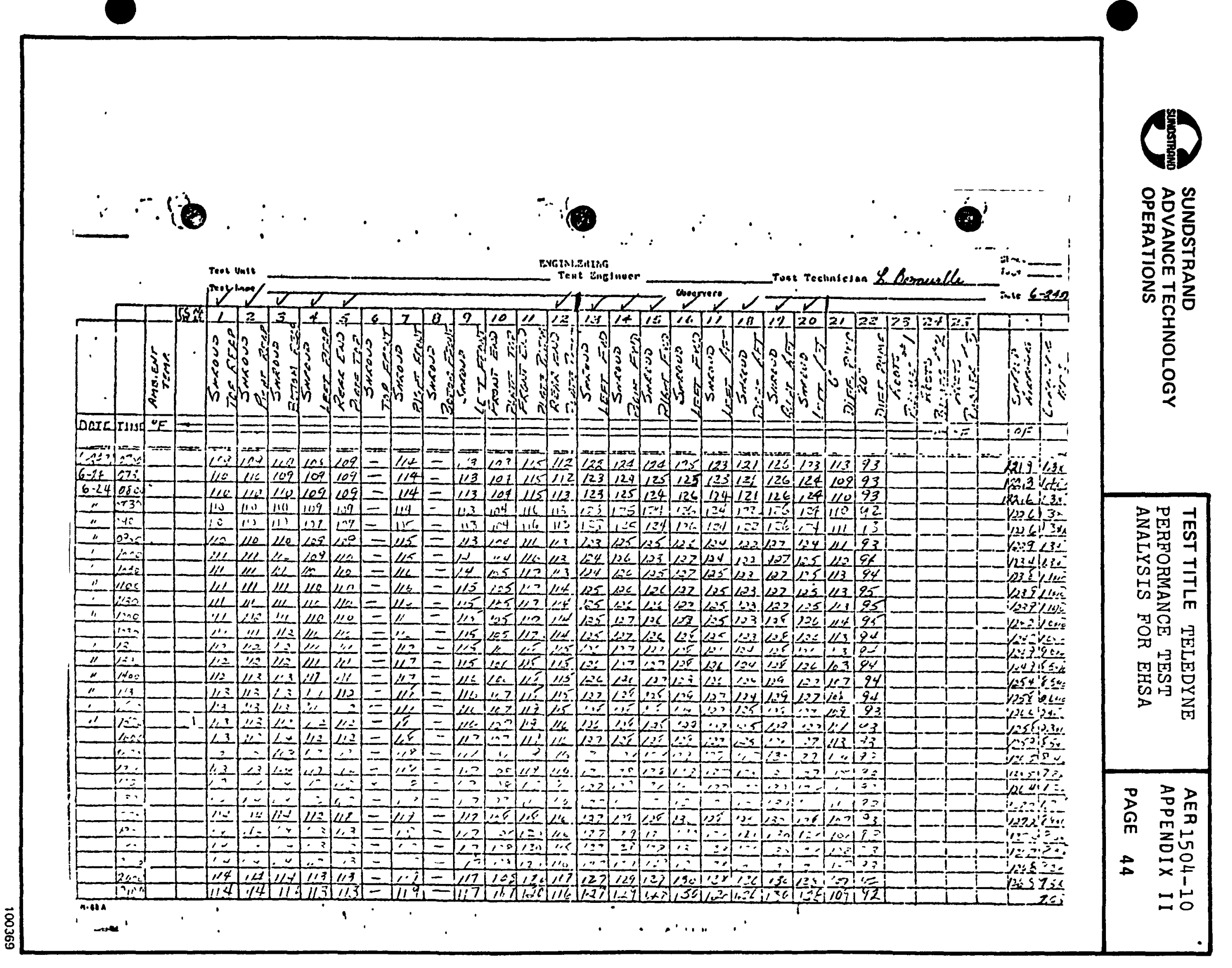




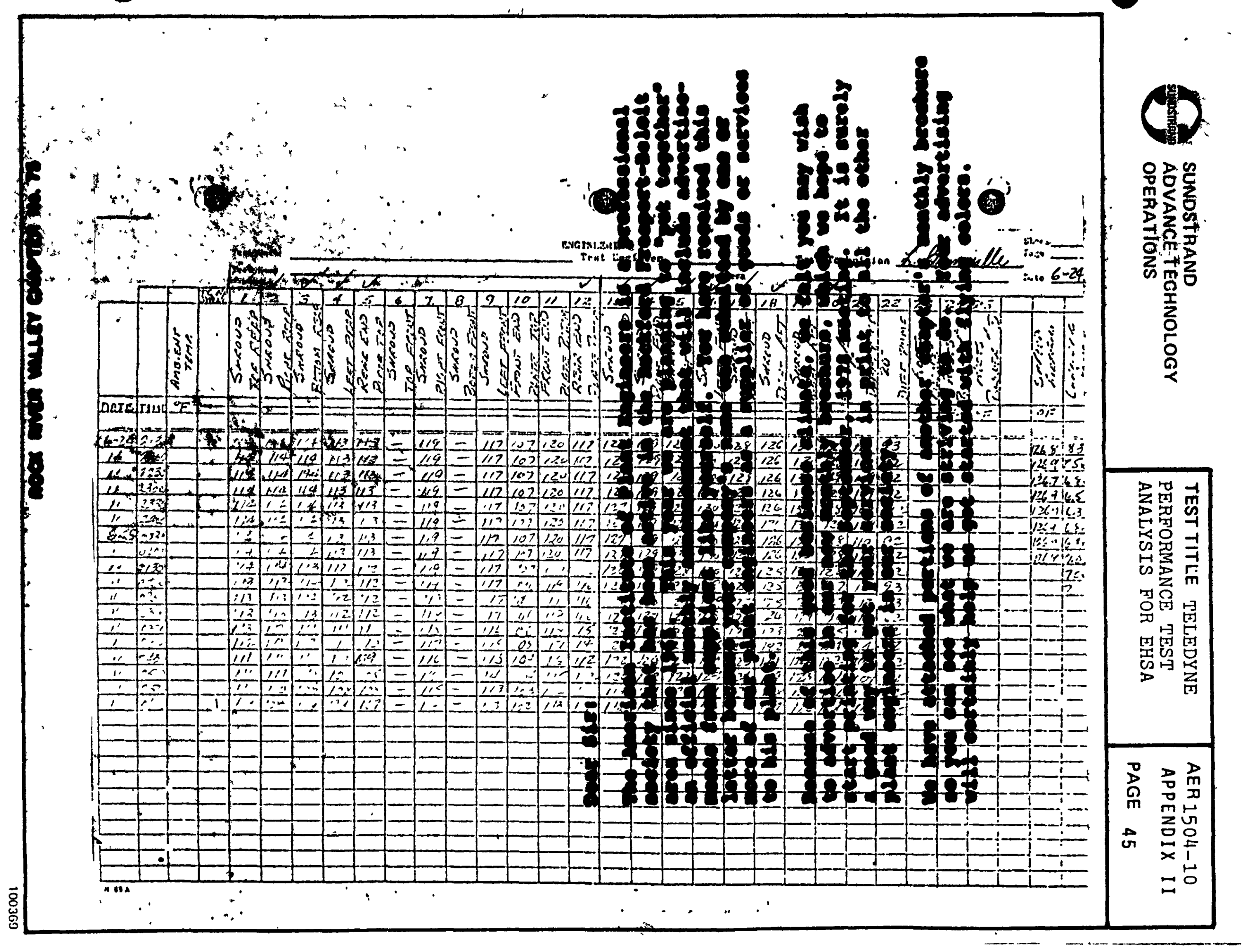

